J. Korean Math. Soc. 52 (2015), No. 2, pp. 269-331

http://dx.doi.org/10.4134/JKMS.2015.52.2.269

\title{
POLARIZED REAL TORI
}

\author{
JAE-HYUN YANG
}

\begin{abstract}
For a fixed positive integer $g$, we let $\mathcal{P}_{g}=\left\{Y \in \mathbb{R}^{(g, g)} \mid Y=\right.$ $\left.{ }^{t} Y>0\right\}$ be the open convex cone in the Euclidean space $\mathbb{R}^{g(g+1) / 2}$. Then the general linear group $G L(g, \mathbb{R})$ acts naturally on $\mathcal{P}_{g}$ by $A \star Y=A Y^{t} A$ $\left(A \in G L(g, \mathbb{R}), Y \in \mathcal{P}_{g}\right)$. We introduce a notion of polarized real tori. We show that the open cone $\mathcal{P}_{g}$ parametrizes principally polarized real tori of dimension $g$ and that the Minkowski modular space $\mathfrak{T}_{g}=G L(g, \mathbb{Z}) \backslash \mathcal{P}_{g}$ may be regarded as a moduli space of principally polarized real tori of dimension $g$. We also study smooth line bundles on a polarized real torus by relating them to holomorphic line bundles on its associated polarized real abelian variety.
\end{abstract}

\section{Introduction}

For a given fixed positive integer $g$, we let

$$
\mathbb{H}_{g}=\left\{\Omega \in \mathbb{C}^{(g, g)} \mid \Omega={ }^{t} \Omega, \operatorname{Im} \Omega>0\right\}
$$

be the Siegel upper half plane of degree $g$ and let

$$
S p(g, \mathbb{R})=\left\{M \in \mathbb{R}^{(2 g, 2 g)} \mid{ }^{t} M J_{g} M=J_{g}\right\}
$$

be the symplectic group of degree $g$, where $F^{(k, l)}$ denotes the set of all $k \times l$ matrices with entries in a commutative ring $F$ for two positive integers $k$ and $l,{ }^{t} M$ denotes the transpose matrix of a matrix $M$ and

$$
J_{g}=\left(\begin{array}{cc}
0 & I_{g} \\
-I_{g} & 0
\end{array}\right) .
$$

Then $S p(g, \mathbb{R})$ acts on $\mathbb{H}_{g}$ transitively by

$$
M \cdot \Omega=(A \Omega+B)(C \Omega+D)^{-1},
$$

Received January 23, 2014.

2010 Mathematics Subject Classification. Primary 14K10.

Key words and phrases. polarized real tori, line bundles over a real torus, semi-abelian varieties, semi-tori.

This work was supported by Basic Science Program through the National Research Foundation of Korea(NRF) funded by the Ministry of Education, Science and Technology (4333801) and partially supported by the Max-Planck-Institut für Mathematik in Bonn. 
where $M=\left(\begin{array}{cc}A & B \\ C & D\end{array}\right) \in S p(g, \mathbb{R})$ and $\Omega \in \mathbb{H}_{g}$. Let

$$
\Gamma_{g}=S p(g, \mathbb{Z})=\left\{\left(\begin{array}{ll}
A & B \\
C & D
\end{array}\right) \in S p(g, \mathbb{R}) \mid A, B, C, D \text { integral }\right\}
$$

be the Siegel modular group of degree $g$. This group acts on $\mathbb{H}_{g}$ properly discontinuously. C. L. Siegel investigated the geometry of $\mathbb{H}_{g}$ and automorphic forms on $\mathbb{H}_{g}$ systematically. Siegel [23] found a fundamental domain $\mathcal{F}_{g}$ for $\Gamma_{g} \backslash \mathbb{H}_{g}$ and described it explicitly. Moreover he calculated the volume of $\mathcal{F}_{g}$. We also refer to $[10,14,23]$ for some details on $\mathcal{F}_{g}$. The Siegel modular variety $\mathcal{A}_{g}:=\Gamma_{g} \backslash \mathbb{H}_{g}$ is one of the important arithmetic varieties in the sense that it is regarded as the moduli of principally polarized abelian varieties of dimension $g$. Suggested by Siegel, I. Satake [18] found a canonical compactification, now called the Satake compactification of $\mathcal{A}_{g}$. Thereafter W. Baily [3] proved that the Satake compactification of $\mathcal{A}_{g}$ is a normal projective variety. This work was generalized to bounded symmetric domains by W. Baily and A. Borel [4] around the 1960s. Some years later a theory of smooth compactification of bounded symmetric domains was developed by Mumford school [2]. G. Faltings and C.-L. Chai [7] investigated the moduli of abelian varieties over the integers and could give the analogue of the Eichler-Shimura theorem that expresses Siegel modular forms in terms of the cohomology of local systems on $\mathcal{A}_{g}$. I want to emphasize that Siegel modular forms play an important role in the theory of the arithmetic and the geometry of the Siegel modular variety $\mathcal{A}_{g}$.

We let

$$
\mathcal{P}_{g}=\left\{Y \in \mathbb{R}^{(g, g)} \mid Y={ }^{t} Y>0\right\}
$$

be an open convex cone in $\mathbb{R}^{N}$ with $N=g(g+1) / 2$. The general linear group $G L(g, \mathbb{R})$ acts on $\mathcal{P}_{g}$ transitively by

$$
A \circ Y:=A Y^{t} A, \quad A \in G L(g, \mathbb{R}), Y \in \mathcal{P}_{g} .
$$

We observe that the action (1.2) is naturally induced from the symplectic action (1.1). Thus $\mathcal{P}_{g}$ is a symmetric space diffeomorphic to $G L(g, \mathbb{R}) / O(g)$. Let

$$
G L(g, \mathbb{Z})=\{\gamma \in G L(g, \mathbb{R}) \mid \gamma \text { is integral }\}
$$

be an arithmetic discrete subgroup of $G L(g, \mathbb{R})$. Using the reduction theory Minkowski [16] found a fundamental domain $\mathfrak{R}_{g}$, the so-called Minkowski fundamental domain for the action $(1.2)$ of $G L(g, \mathbb{Z})$ on $\mathcal{P}_{g}$. In fact, using the Minkowski fundamental domain $\mathfrak{R}_{g}$ Siegel found his fundamental domain $\mathcal{F}_{g}$. As in the case of $\mathbb{H}_{g}$, automorphic forms on $\mathcal{P}_{g}$ for $G L(g, \mathbb{Z})$ and geometry on $\mathcal{P}_{g}$ have been studied by many people, e.g., Selberg [20], Maass [14] et al.

The aim of this article is to study arithmetic-geometric meaning of the Minkowski fundamental domain $\mathfrak{R}_{g}$. First we introduce a notion of polarized real tori by relating special real tori to polarized real abelian varieties. We realize that $\mathcal{P}_{g}$ parametrizes principally polarized real tori of dimension $g$ and also that the Minkowski modular space $\mathfrak{T}_{g}:=G L(g, \mathbb{Z}) \backslash \mathcal{P}_{g}$ may be regarded as a moduli space of principally polarized real tori of dimension $g$. We also study 
smooth line bundles over a polarized real torus by relating to holomorphic line bundles over the associated polarized abelian variety. Those line bundles over a polarized real torus play an important role in investigating some geometric properties of a polarized real torus.

We let

$$
G^{M}:=G L(g, \mathbb{R}) \ltimes \mathbb{R}^{g}
$$

be the semidirect product of $G L(g, \mathbb{R})$ and $\mathbb{R}^{g}$ with multiplication law

$$
(A, a) \cdot(B, b):=\left(A B, a^{t} B^{-1}+b\right), \quad A, B \in G L(g, \mathbb{R}), \quad a, b \in \mathbb{R}^{g} .
$$

Then we have the natural action of $G^{M}$ on the Minkowski-Euclid space $\mathcal{P}_{g} \times \mathbb{R}^{g}$ defined by

$$
(A, a) \cdot(Y, \zeta):=\left(A Y^{t} A,(\zeta+a)^{t} A\right), \quad(A, a) \in G^{M}, Y \in \mathcal{P}_{g}, \zeta \in \mathbb{R}^{g} .
$$

We let

$$
G^{M}(\mathbb{Z})=G L(g, \mathbb{Z}) \ltimes \mathbb{Z}^{g}
$$

be the discrete subgroup of $G^{M}$. Then $G^{M}(\mathbb{Z})$ acts on $\mathcal{P}_{g} \times \mathbb{R}^{g}$ properly discontinuously. We show that by associating a principally polarized real torus of dimension $g$ to each equivalence class in $\mathfrak{T}_{g}$, the quotient space

$$
G^{M}(\mathbb{Z}) \backslash\left(\mathcal{P}_{g} \times \mathbb{R}^{g}\right)
$$

may be regarded as a family of principally polarized real tori of dimension g. To each equivalence class $[Y] \in G L(g, \mathbb{Z}) \backslash \mathcal{P}_{g}$ with $Y \in \mathcal{P}_{g}$ we associate a principally polarized real torus $T_{Y}=\mathbb{R}^{g} / \Lambda_{Y}$, where $\Lambda_{Y}=Y \mathbb{Z}^{g}$ is a lattice in $\mathbb{R}^{g}$.

Let $Y_{1}$ and $Y_{2}$ be two elements in $\mathcal{P}_{g}$ with $\left[Y_{1}\right] \neq\left[Y_{2}\right]$, that is, $Y_{2} \neq A Y_{1}{ }^{t} A$ for all $A \in G L(g, \mathbb{Z})$. We put $\Lambda_{i}=Y_{i} \mathbb{Z}^{g}$ for $i=1,2$. Then a torus $T_{1}=\mathbb{R}^{g} / \Lambda_{1}$ is diffeomorphic to $T_{2}=\mathbb{R}^{g} / \Lambda_{2}$ as smooth manifolds but $T_{1}$ is not isomorphic to $T_{2}$ as polarized real tori.

The Siegel modular variety $\mathcal{A}_{g}$ has three remarkable properties: (a) it is the moduli space of principally polarized abelian varieties of dimension $g$, (b) it has the structure of a quasi-projective complex algebraic variety which is defined over $\mathbb{Q}$, and (c) it has a canonical compactification, the so-called Satake-BailyBorel compactification which is defined over $\mathbb{Q}$. Unfortunately the Minkowski modular space $\mathfrak{T}_{g}$ does not admit the structure of a real algebraic variety. Moreover $\mathfrak{T}_{g}$ does not admit a compactification which is defined over $\mathbb{Q}$. Silhol [26] constructs the moduli space of real principally poarized abelian varieties and he shows that it is a topological ramified covering of $\mathfrak{T}_{g}$. Furthermore Silhol constructs a compactification of this moduli space analogous to the Satake-Baily-Borel compactification. However, neither the moduli space nor this compactification has an algebraic structure. On the other hand, by considering real abelian varieties with a suitable level structure Goresky and Tai [8] show that the moduli space of real principally polarized abelian varieties with level $4 m$ structure $(m \geq 1)$ coincides with the set of real points of a quasi-projective algebraic variety defined over $\mathbb{Q}$ and consists of finitely 
many copies of the quotient $\mathfrak{G}_{g}(4 m) \backslash \mathcal{P}_{g}$ with a discrete subgroup $\mathfrak{G}_{g}(4 m)$ of $G L(g, \mathbb{Z})$, where $\mathfrak{G}_{g}(4 m)=\left\{\gamma \in G L(g, \mathbb{Z}) \mid \gamma \equiv I_{g}(\bmod 4 m)\right\}$.

This paper is organized as follows. In Section 2, we collect some basic properties about the symplectic group $S p(g, \mathbb{R})$ to be used frequently in the subsequent sections. In Section 3, we give basic definitions concerning real abelian varieties and review some properties of real abelian varieties. In Section 4, we discuss a moduli space for real abelian varieties and recall some basic properties of a moduli for real abelian varieties. In Section 5 we discuss compactifications of the moduli space for real abelian varieties and review some results on this moduli space obtained by Silhol [26], Goresky and Tai [8]. In Section 6 we introduce a notion of polarized real tori and investigate some properties of polarized real tori. We give several examples of polarized real tori. In Section 7 we study smooth line bundles over a real torus, in particular a polarized real torus by relating those smooth line bundles to holomorphic line bundles over the associated complex torus. To each smooth line bundle on a real torus we naturally attach a holomorphic line bundle over the associated complex torus. Conversely to a holomorphic line bundle over a polarized abelian variety we associate a smooth line bundle over the associated polarized real torus. Using these results on line bundles, we embed a real torus in a complex projective space and hence in a real projective space smoothly. We also review briefly holomorphic line bundles over a complex torus. In Section 8 we study the moduli space for polarized real tori. We first review basic geometric properties on the Minkowski fundamental domain $\mathfrak{R}_{g}$. We show that $\mathcal{P}_{g}$ parameterizes principally polarized real tori of dimension $g$ and that $\mathfrak{T}_{g}$ can be regarded as the moduli space of principally polarized real tori of dimension $g$. We show that the quotient space $G^{M}(\mathbb{Z}) \backslash\left(\mathcal{P}_{g} \times \mathbb{R}^{g}\right)$ may be considered as a family of principally polarized real tori of dimension $g$. In Section 9 we discuss real semiabelian varieties corresponding to the boundary points of a compactification of a moduli space for real abelian varieties. We recall that a semi-abelian variety is defined to be an extension of an abelian variety by a group of multiplicative type. In Section 10 we discuss briefly real semi-tori corresponding to the boundary points of a moduli space for polarized real tori. In the final section we present some problems related to real polarized tori which should be investigated in the near future. In the appendix we collect and review some results on non-abelian cohomology to be needed necessarily in this article. We give some sketchy proofs for the convenience of the reader.

Finally I would like to mention that this work was motivated and initiated by the works of Silhol [26] and Goresky-Tai [8].

Notations. We denote by $\mathbb{Q}, \mathbb{R}$ and $\mathbb{C}$ the field of rational numbers, the field of real numbers and the field of complex numbers respectively. We denote by $\mathbb{Z}$ and $\mathbb{Z}^{+}$the ring of integers and the set of all positive integers respectively. The symbol ":=" means that the expression on the right is the definition of that on the left. For two positive integers $k$ and $l, F^{(k, l)}$ denotes the set of all $k \times l$ 
matrices with entries in a commutative ring $F$. For a square matrix $A \in F^{(k, k)}$ of degree $k, \sigma(A)$ denotes the trace of $A$. For any $M \in F^{(k, l)},{ }^{t} M$ denotes the transpose matrix of $M . I_{n}$ denotes the identity matrix of degree $n$. For a matrix $Z$, we denote by $\operatorname{Re} Z$ (resp. $\operatorname{Im} Z$ ) the real (resp. imaginary) part of $Z$. For $A \in F^{(k, l)}$ and $B \in F^{(k, k)}$, we set $B[A]={ }^{t} A B A$. For a complex matrix $A, \bar{A}$ denotes the complex conjugate of $A$. For $A \in \mathbb{C}^{(k, l)}$ and $B \in \mathbb{C}^{(k, k)}$, we use the abbreviation $B\{A\}={ }^{t} \bar{A} B A$. We denote $\mathbb{C}_{1}^{*}=\{\xi \in \mathbb{C}|| \xi \mid=1\}$. Let

$$
\Gamma_{g}=\left\{\gamma \in \mathbb{Z}^{(2 g, 2 g)} \mid{ }^{t} \gamma J_{g} \gamma=J_{g}\right\}
$$

denote the Siegel modular group of degree $g$, where

$$
J_{g}=\left(\begin{array}{cc}
0 & I_{g} \\
-I_{g} & 0
\end{array}\right)
$$

is the symplectic matrix of degree $2 g$. For a positive integer $N$, we let

$$
\Gamma_{g}(N)=\left\{\gamma \in \Gamma_{g} \mid \gamma \equiv I_{2 g}(\bmod N)\right\}
$$

denote the principal congruence subgroup of $\Gamma_{g}$ of level $N$ and for a positive integer $m$, we let

$$
\Gamma_{g}(2,2 m)=\left\{\left(\begin{array}{cc}
A & B \\
C & D
\end{array}\right) \in \Gamma_{g} \mid A, D \equiv I_{g}(\bmod 2), \quad B, C \equiv 0(\bmod 2 m)\right\} .
$$

Let $\mathfrak{G}_{g}:=G L(g, \mathbb{Z})$ and for a positive integer $N$ let

$$
\mathfrak{G}_{g}(N)=\left\{\gamma \in G L(g, \mathbb{Z}) \mid \gamma \equiv I_{g}(\bmod N)\right\} .
$$

\section{The symplectic group}

For a given fixed positive integer $g$, we let let

$$
S p(g, \mathbb{R})=\left\{M \in \mathbb{R}^{(2 g, 2 g)} \mid{ }^{t} M J_{g} M=J_{g}\right\}
$$

be the symplectic group of degree $g$.

If $M=\left(\begin{array}{cc}A & B \\ C & D\end{array}\right) \in S p(g, \mathbb{R})$ with $A, B, C, D \in \mathbb{R}^{(g, g)}$, then it is easily seen that

$$
A^{t} D-B^{t} C=I_{g}, \quad A^{t} B=B^{t} A, \quad C^{t} D=D^{t} C
$$

or

$$
{ }^{t} A D-{ }^{t} C B=I_{g}, \quad{ }^{t} A C={ }^{t} C A, \quad{ }^{t} B D={ }^{t} D B .
$$

The inverse of such a symplectic matrix $M$ is given by

$$
M^{-1}=M=\left(\begin{array}{cc}
{ }^{t} D & -{ }^{t} B \\
-{ }^{t} C & { }^{t} A
\end{array}\right) .
$$

We identify $G L(g, \mathbb{R}) \hookrightarrow S p(g, \mathbb{R})$ with its image under the embedding

$$
A \longmapsto\left(\begin{array}{cc}
A & 0 \\
0 & { }^{t} A^{-1}
\end{array}\right), \quad A \in G L(g, \mathbb{R}) .
$$


A Cartan involution $\theta$ of $S p(g, \mathbb{R})$ is given by $\theta(x)=J_{g} x J_{g}^{-1}, x \in S p(g, \mathbb{R})$, in other words,

$$
\theta\left(\begin{array}{ll}
A & B \\
C & D
\end{array}\right)=\left(\begin{array}{cc}
D & -C \\
-B & A
\end{array}\right), \quad\left(\begin{array}{ll}
A & B \\
C & D
\end{array}\right) \in S p(g, \mathbb{R}) .
$$

The fixed point set $K$ of $\theta$ is given by

$$
K=\left\{\left(\begin{array}{cc}
A & B \\
-B & A
\end{array}\right) \in S p(g, \mathbb{R})\right\} .
$$

We may identify $K$ with the unitary group $U(g)$ of degree $g$ via

$$
K \ni\left(\begin{array}{cc}
A & B \\
-B & A
\end{array}\right) \longmapsto A+i B \in U(g) .
$$

Let

$$
\mathbb{H}_{g}=\left\{\Omega \in \mathbb{C}^{(g, g)} \mid \Omega={ }^{t} \Omega, \operatorname{Im} \Omega>0\right\}
$$

be the Siegel upper half plane of degree $g$. Then $S p(g, \mathbb{R})$ acts on $\mathbb{H}_{g}$ transitively by

$$
M \cdot \Omega=(A \Omega+B)(C \Omega+D)^{-1},
$$

where $M=\left(\begin{array}{ll}A & B \\ C & D\end{array}\right) \in S p(g, \mathbb{R})$ and $\Omega \in \mathbb{H}_{g}$. The stabilizer at $i I_{g}$ is given by the compact subgroup $K \cong U(g)$ of $S p(g, \mathbb{R})$. Thus $\mathbb{H}_{g}$ is biholomorphic to the Hermitian symmetric space $S p(g, \mathbb{R}) / K$ via

$$
S p(g, \mathbb{R}) / K \longrightarrow \mathbb{H}_{g}, \quad x K \longmapsto x \cdot\left(i I_{g}\right), x \in S p(g, \mathbb{R}) .
$$

We note that the Siegel modular group $\Gamma_{g}$ of degree $g$ acts on $\mathbb{H}_{g}$ properly discontinuously.

Now we let

$$
I_{*}:=\left(\begin{array}{cc}
-I_{g} & 0 \\
0 & I_{g}
\end{array}\right) .
$$

We define the involution $\tau: S p(g, \mathbb{R}) \longrightarrow S p(g, \mathbb{R})$ by

$$
\tau(x):=I_{*} x I_{*}, \quad x \in S p(g, \mathbb{R}) .
$$

Precisely $\tau$ is given by

$$
\tau\left(\begin{array}{ll}
A & B \\
C & D
\end{array}\right)=\left(\begin{array}{cc}
A & -B \\
-C & D
\end{array}\right), \quad\left(\begin{array}{ll}
A & B \\
C & D
\end{array}\right) \in S p(g, \mathbb{R}) .
$$

Lemma 2.1. (1) $\tau(x)=x, x \in S p(g, \mathbb{R})$ if and only if $x \in G L(g, \mathbb{R})$.

(2) $\tau \theta=\theta \tau$. So $\tau(K)=K$.

(3) If $A+i B \in U(g)$ with $A, B \in \mathbb{R}^{(g, g)}$, then $\tau(A+i B)=A-i B$.

Proof. It is easy to prove the above lemma. We leave the proof to the reader. 
We note that $\tau: S p(g, \mathbb{R}) \longrightarrow S p(g, \mathbb{R})$ passes to an involution (which we denote by the same letter) $\tau: \mathbb{H}_{g} \longrightarrow \mathbb{H}_{g}$ such that

$$
\tau(x \cdot \Omega)=\tau(x) \tau(\Omega) \quad \text { for all } x \in S p(g, \mathbb{R}), \Omega \in \mathbb{H}_{g} .
$$

In fact, we can see easily that the involution $\tau: \mathbb{H}_{g} \longrightarrow \mathbb{H}_{g}$ is the antiholomorphic involution given by

$$
\tau(\Omega)=-\bar{\Omega}, \quad \Omega \in \mathbb{H}_{g} .
$$

Its fixed point set is the orbit

$$
i \mathcal{P}_{g}=G L(g, \mathbb{R}) \cdot\left(i I_{g}\right) \subset \mathbb{C}^{(g, g)}
$$

of $G L(g, \mathbb{R})$, where

$$
\mathcal{P}_{g}=\left\{Y \in \mathbb{R}^{(g, g)} \mid Y={ }^{t} Y>0\right\}
$$

is the open convex cone of positive definite symmetric real matrices of degree $g$ in the Euclidean space $\mathbb{R}^{g(g+1) / 2}$.

For $x \in S p(g, \mathbb{R})$ and $\Omega \in \mathbb{H}_{g}$, we define the set

$$
\mathbb{H}_{g}^{\tau x}:=\left\{\Omega \in \mathbb{H}_{g} \mid x \cdot \Omega=\tau(\Omega)=-\bar{\Omega}\right\}
$$

be the locus of $x$-real points. If $\Gamma \subset S p(g, \mathbb{R})$ is an arithmetic subgroup of $\operatorname{Sp}(g, \mathbb{R})$ such that $\tau(\Gamma)=\Gamma$, we define

$$
\mathbb{H}_{g}^{\tau \Gamma}:=\bigcup_{\gamma \in \Gamma} \mathbb{H}_{g}^{\tau \gamma}
$$

Lemma 2.2. Let $x \in S p(g, \mathbb{R})$ and $\mathbb{H}_{g}^{x}$ be the set of points in $\mathbb{H}_{g}$ which are fixed under the action of $x$. Then the set $\mathbb{H}_{g}^{x} \cap i \mathcal{P}_{g}$ is a proper real algebraic variety of $i \mathcal{P}_{g}$ if $x \neq \pm I_{g} \in G L(g, \mathbb{R})$.

Proof. It is easy to prove the above lemma. We omit the proof.

\section{Real abelian varieties}

In this section we review basic notions and some results on real principally polarized abelian varieties (cf. [8, 21, 24, 25, 26]).

Definition. A pair $(\mathfrak{A}, S)$ is said to be a real abelian variety if $\mathfrak{A}$ is a complex abelian variety and $S$ is an anti-holomorphic involution of $\mathfrak{A}$ leaving the origin of $\mathfrak{A}$ fixed. The set of all fixed points of $S$ is called the real point of $(\mathfrak{A}, S)$ and denoted by $(\mathfrak{A}, S)(\mathbb{R})$ or simply $\mathfrak{A}(\mathbb{R})$. We call $S$ a real structure on $\mathfrak{A}$.

Definition. (1) A polarization on a complex abelian variety $\mathfrak{A}$ is defined to be the Chern class $c_{1}(D) \in H^{2}(\mathfrak{A}, \mathbb{Z})$ of an ample divisor $D$ on $\mathfrak{A}$. We can identify $H^{2}(\mathfrak{A}, \mathbb{Z})$ with $\bigwedge^{2} H^{1}(\mathfrak{A}, \mathbb{Z})$. We write $\mathfrak{A}=V / L$, where $V$ is a finite dimensional complex vector space and $L$ is a lattice in $V$. So a polarization on $\mathfrak{A}$ can be defined as an alternating form $E$ on $L \cong H_{1}(\mathfrak{A}, \mathbb{Z})$ satisfying the following conditions (E1) and (E2): 
(E1) The Hermitian form $H: V \times V \longrightarrow \mathbb{C}$ defined by

$$
H(u, v)=E(i u, v)+i E(u, v), \quad u, v \in V
$$

is positive definite. Here $E$ can be extended $\mathbb{R}$-linearly to an alternating form on $V$.

(E2) $E(L \times L) \subset \mathbb{Z}$, i.e., $E$ is integral valued on $L \times L$.

(2) Let $(\mathfrak{A}, S)$ be a real abelian variety with a polarization $E$ of dimension g. A polarization $E$ is said to be real or $S$-real if

$$
E\left(S_{*}(a), S_{*}(b)\right)=-E(a, b), \quad a, b \in H_{1}(\mathfrak{A}, \mathbb{Z}) .
$$

Here $S_{*}: H_{1}(\mathfrak{A}, \mathbb{Z}) \longrightarrow H_{1}(\mathfrak{A}, \mathbb{Z})$ is the map induced by a real structure $S$. If a polarization $E$ is real, the triple $(\mathfrak{A}, E, S)$ is called a real polarized abelian variety. A polarization $E$ on $\mathfrak{A}$ is said to be principal if for a suitable basis (i.e., a symplectic basis) of $H_{1}(\mathfrak{A}, \mathbb{Z}) \cong L$, it is represented by the symplectic matrix $J_{g}$ (cf. see Notations in the introduction). A real abelian variety $(\mathfrak{A}, S)$ with a principal polarization $E$ is called a real principally polarized abelian variety.

(3) Let $(\mathfrak{A}, E)$ be a principally polarized abelian variety of dimension $g$ and let $\left\{\alpha_{i} \mid 1 \leq i \leq 2 g\right\}$ be a symplectic basis of $H_{1}(\mathfrak{A}, \mathbb{Z})$. It is known that there is a basis $\left\{\omega_{1}, \ldots, \omega_{g}\right\}$ of the vector space $H^{0}\left(\mathfrak{A}, \Omega^{1}\right)$ of holomorphic 1-forms on $\mathfrak{A}$ such that

$$
\left(\int_{\alpha_{j}} \omega_{i}\right)=\left(\Omega, I_{g}\right) \quad \text { for some } \Omega \in \mathbb{H}_{g} .
$$

The $g \times 2 g$ matrix $\left(\Omega, I_{g}\right)$ or simply $\Omega$ is called a period matrix for $(\mathfrak{A}, E)$.

The definition of a real polarized abelian variety is motivated by the following theorem.

Theorem 3.1. Let $(\mathfrak{A}, S)$ be a real abelian variety and let $E$ be a polarization on $\mathfrak{A}$. Then there exists an ample $S$-invariant (or $S$-real) divisor with Chern class $E$ if and only if $E$ satisfies the condition (3.2).

Proof. The proof can be found in [25, Theorem 3.4, pp. 81-84].

Now we consider a principally polarized abelian variety of dimension $g$ with a level structure. Let $N$ be a positive integer. Let $\left(\mathfrak{A}=\mathbb{C}^{g} / L, E\right)$ be a principally polarized abelian variety of dimension $g$. From now on we write $\mathfrak{A}=\mathbb{C}^{g} / L$, where $L$ is a lattice in $\mathbb{C}^{g}$. A level $N$ structure on $\mathfrak{A}$ is a choice of a basis $\left\{U_{i}, V_{j}\right\}(1 \leq i, j \leq g)$ for a $N$-torsion points of $\mathfrak{A}$ which is symplectic, in the sense that there exists a symplectic basis $\left\{u_{i}, v_{j}\right\}$ of $L$ such that

$$
U_{i} \equiv \frac{u_{i}}{N}(\bmod L) \quad \text { and } \quad V_{j} \equiv \frac{v_{j}}{N}(\bmod L), \quad 1 \leq i, j \leq g .
$$

For a given level $N$ structure, such a choice of a symplectic basis $\left\{u_{i}, v_{j}\right\}$ of $L$ determines a mapping

$$
F: \mathbb{R}^{g} \oplus \mathbb{R}^{g} \longrightarrow \mathbb{C}^{g}
$$


such that $F\left(\mathbb{Z}^{g} \oplus \mathbb{Z}^{g}\right)=L$ by $F\left(e_{i}\right)=u_{i}$ and $F\left(f_{j}\right)=v_{j}$, where $\left\{e_{i}, f_{j}\right\}(1 \leq$ $i, j \leq g)$ is the standard basis of $\mathbb{R}^{g} \oplus \mathbb{R}^{g}$. The choice $\left\{u_{i}, v_{j}\right\}$ (or equivalently, the mapping $F$ ) will be referred to as a lift of the level $N$ structure. Such a mapping $F$ is well defined modulo the principal congruence subgroup $\Gamma_{g}(N)$, that is, if $F^{\prime}$ is another lift of the level structure, then $F^{\prime} \circ F^{-1} \in \Gamma_{g}(N)$. A level $N$ structure $\left\{U_{i}, V_{j}\right\}$ is said to be compatible with a real structure $S$ on $(\mathfrak{A}, E)$ if, for some (and hence for any) lift $\left\{u_{i}, v_{j}\right\}$ of the level structure,

$$
S\left(\frac{u_{i}}{N}\right) \equiv-\frac{u_{i}}{N}(\bmod L) \quad \text { and } \quad S\left(\frac{v_{j}}{N}\right) \equiv \frac{v_{j}}{N}(\bmod L), \quad 1 \leq i, j \leq g .
$$

Definition. A real principally polarized abelian variety of dimension $g$ with a level $N$ structure is a quadruple $\mathcal{A}=\left(\mathfrak{A}, E, S,\left\{U_{i}, V_{j}\right\}\right)$ with $\mathfrak{A}=\mathbb{C}^{g} / L$, where $(\mathfrak{A}, E, S)$ is a real principally polarized abelian variety and $\left\{U_{i}, V_{j}\right\}$ is a level $N$ structure compatible with a real structure $S$. An isomorphism

$$
\mathcal{A}=\left(\mathfrak{A}, E, S,\left\{U_{i}, V_{j}\right\}\right) \cong\left(\mathfrak{A}^{\prime}, E^{\prime}, S^{\prime},\left\{U_{i}^{\prime}, V_{j}^{\prime}\right\}\right)=\mathcal{A}^{\prime}
$$

is a complex linear mapping $\phi: \mathbb{C}^{g} \longrightarrow \mathbb{C}^{g}$ such that

$$
\begin{gathered}
\phi(L)=L^{\prime}, \\
\phi_{*}(E)=E^{\prime}, \\
\phi_{*}(S)=S^{\prime}, \text { that is, } \phi \circ S \circ \phi^{-1}=S^{\prime}, \\
\phi\left(\frac{u_{i}}{N}\right) \equiv \frac{u_{i}^{\prime}}{N}\left(\bmod L^{\prime}\right) \text { and } \phi\left(\frac{v_{j}}{N}\right) \equiv \frac{v_{j}^{\prime}}{N}\left(\bmod L^{\prime}\right), \quad 1 \leq i, j \leq g
\end{gathered}
$$

for some lift $\left\{u_{i}, v_{j}\right\}$ and $\left\{u_{i}^{\prime}, v_{j}^{\prime}\right\}$ of the level structures.

Now we show that a given positive integer $N$ and a given $\Omega \in \mathbb{H}_{g}$ determine naturally a principally polarized abelian variety $\left(\mathfrak{A}_{\Omega}, E_{\Omega}\right)$ of dimension $g$ with a level $N$ structure. Let $E_{0}$ be the standard alternating form on $\mathbb{R}^{g} \oplus \mathbb{R}^{g}$ with the symplectic matrix $J_{g}$ with respect to the standard basis of $\mathbb{R}^{g} \oplus \mathbb{R}^{g}$. Let $F_{\Omega}: \mathbb{R}^{g} \oplus \mathbb{R}^{g} \longrightarrow \mathbb{C}^{g}$ be the real linear mapping with matrix $\left(\Omega, I_{g}\right)$, that is,

$$
F_{\Omega}\left(\begin{array}{l}
x \\
y
\end{array}\right):=\Omega x+y, \quad x, y \in \mathbb{R}^{g} .
$$

We define $E_{\Omega}:=\left(F_{\Omega}\right)_{*}\left(E_{0}\right)$ and $L_{\Omega}:=F_{\Omega}\left(\mathbb{Z}^{g} \oplus \mathbb{Z}^{g}\right)$. Then $\left(\mathfrak{A}_{\Omega}=\mathbb{C}^{g} / L_{\Omega}, E_{\Omega}\right)$ is a principally polarized abelian variety. The Hermitian form $H_{\Omega}$ on $\mathbb{C}^{g}$ corresponding to $E_{\Omega}$ is given by

$$
H_{\Omega}(u, v)={ }^{t} u(\operatorname{Im} \Omega)^{-1} \bar{v}, \quad E_{\Omega}=\operatorname{Im} H_{\Omega}, \quad u, v \in \mathbb{C}^{g} .
$$

If $z_{1}, \ldots, z_{g}$ are the standard coordinates on $\mathbb{C}^{g}$, then the holomorphic 1 -forms $d z_{1}, \ldots, d z_{g}$ have the period matrix $\left(\Omega, I_{g}\right)$. If $\left\{e_{i}, f_{j}\right\}$ is the standard basis of $\mathbb{R}^{g} \oplus \mathbb{R}^{g}$, then $\left\{F_{\Omega}\left(e_{i} / N\right), F_{\Omega}\left(f_{j} / N\right)\right\}$. 
$\left(\bmod L_{\Omega}\right)$ is a level $N$ structure on $\left(\mathfrak{A}_{\Omega}, E_{\Omega}\right)$, which we refer to as the standard $N$ structure. Assume that $\Omega_{1}$ and $\Omega_{2}$ are two elements of $\mathbb{H}_{g}$ such that

$$
\psi:\left(\mathfrak{A}_{\Omega_{1}}=\mathbb{C}^{g} / L_{\Omega_{1}}, E_{\Omega_{1}}\right) \longrightarrow\left(\mathfrak{A}_{\Omega_{2}}=\mathbb{C}^{g} / L_{\Omega_{2}}, E_{\Omega_{2}}\right)
$$

is an isomorphism of the corresponding principally polarized abelian varieties, i.e., $\psi\left(L_{\Omega_{1}}\right)=L_{\Omega_{2}}$ and $\psi_{*}\left(E_{\Omega_{1}}\right)=E_{\Omega_{2}}$. We set

$$
h={ }^{t}\left(F_{\Omega_{2}}^{-1} \circ \psi \circ F_{\Omega_{1}}\right)=\left(\begin{array}{ll}
A & B \\
C & D
\end{array}\right) .
$$

Then we see that $h \in \Gamma_{g}$. And we have

$$
\Omega_{1}=h \cdot \Omega_{2}=\left(A \Omega_{2}+B\right)\left(C \Omega_{2}+D\right)^{-1}
$$

$$
\psi(Z)={ }^{t}\left(C \Omega_{2}+D\right) Z, \quad Z \in \mathbb{C}^{g} .
$$

Let $\Omega \in \mathbb{H}_{g}$ such that $\gamma \cdot \Omega=\tau(\Omega)=-\bar{\Omega}$ for some $\gamma=\left(\begin{array}{cc}A & B \\ C & D\end{array}\right) \in \Gamma_{g}$. We define the mapping $S_{\gamma, \Omega}: \mathbb{C}^{g} \longrightarrow \mathbb{C}^{g}$ by

$$
S_{\gamma, \Omega}(Z):={ }^{t}(C \Omega+D) \bar{Z}, \quad Z \in \mathbb{C}^{g} .
$$

Then we can show that $S_{\gamma, \Omega}$ is a real structure on $\left(\mathfrak{A}_{\Omega}, E_{\Omega}\right)$ which is compatible with the polarization $E_{\Omega}$ (that is, $E_{\Omega}\left(S_{\gamma, \Omega}(u), S_{\gamma, \Omega}(v)\right)=-E_{\Omega}(u, v)$ for all $u, v \in \mathbb{C}^{g}$ ). Indeed according to Comessatti's Theorem (see Theorem 3.1), $S_{\gamma, \Omega}(Z)=\bar{Z}$, i.e., $S_{\gamma, \Omega}$ is a complex conjugation. Therefore we have

$$
E_{\Omega}\left(S_{\gamma, \Omega}(u), S_{\gamma, \Omega}(v)\right)=E_{\Omega}(\bar{u}, \bar{v})=-E_{\Omega}(u, v)
$$

for all $u, v \in \mathbb{C}^{g}$. From now on we write simply $\sigma_{\Omega}=S_{\gamma, \Omega}$.

Theorem 3.2. Let $(\mathfrak{A}, E, S)$ be a real principally polarized abelian variety of dimension $g$. Then there exists $\Omega=X+i Y \in \mathbb{H}_{g}$ such that $2 X \in \mathbb{Z}^{(g, g)}$ and there exists an isomorphism of real principally polarized abelian varieties

$$
(\mathfrak{A}, E, S) \cong\left(\mathfrak{A}_{\Omega}, E_{\Omega}, \sigma_{\Omega}\right),
$$

where $\sigma_{\Omega}$ is a real structure on $\mathfrak{A}_{\Omega}$ induced by a complex conjugation $\sigma: \mathbb{C}^{g} \longrightarrow$ $\mathbb{C}^{g}$.

The above theorem is essentially due to Comessatti [6]. We refer to $[24,25]$ for the proof of Theorem 3.2.

Theorem 3.2 leads us to define the subset $\mathscr{H}_{g}$ of $\mathbb{H}_{g}$ by

$$
\mathscr{H}_{g}:=\left\{\Omega \in \mathbb{H}_{g} \mid 2 \operatorname{Re} \Omega \in \mathbb{Z}^{(g, g)}\right\} .
$$

Assume $\Omega=X+i Y \in \mathscr{H}_{g}$. Then according to Theorem $3.2,\left(\mathfrak{A}_{\Omega}, E_{\Omega}, \sigma_{\Omega}\right)$ is a real principally polarized abelian variety of dimension $g$. The matrix $M_{\sigma}$ for the action of a complex conjugation $\sigma$ on the lattice $L_{\Omega}=\Omega \mathbb{Z}^{g}+\mathbb{Z}^{g}$ with respect to the basis given by the columns of $\left(\Omega, I_{g}\right)$ is given by

$$
M_{\sigma}=\left(\begin{array}{cc}
-I_{g} & 0 \\
2 X & I_{g}
\end{array}\right) .
$$


Since

the canonical polarization $J_{g}$ is $\sigma$-real.

$$
{ }^{t} M_{\sigma} J_{g} M_{\sigma}=\left(\begin{array}{cc}
-I_{g} & 2 X \\
0 & I_{g}
\end{array}\right) J_{g}\left(\begin{array}{cc}
-I_{g} & 0 \\
2 X & I_{g}
\end{array}\right)=-J_{g},
$$

Theorem 3.3. Let $\Omega$ and $\Omega_{*}$ be two elements in $\mathscr{H}_{g}$. Then $\Omega$ and $\Omega_{*}$ represent (real) isomorphic triples $(\mathfrak{A}, E, \sigma)$ and $\left(\mathfrak{A}_{*}, E_{*}, \sigma_{*}\right)$ if and only if there exists an element $A \in G L(g, \mathbb{Z})$ such that

$$
2 \operatorname{Re} \Omega_{*}=2 A(\operatorname{Re} \Omega)^{t} A(\bmod 2)
$$

and

$$
\operatorname{Im} \Omega_{*}=A(\operatorname{Im} \Omega){ }^{t} A .
$$

Proof. Suppose $(\mathfrak{A}, E, \sigma)$ and $\left(\mathfrak{A}_{*}, E_{*}, \sigma_{*}\right)$ are real isomorphic. Then we can find an element $\gamma=\left(\begin{array}{cc}A & B \\ C & D\end{array}\right) \in \Gamma_{g}$ such that

$$
\Omega_{*}=(A \Omega+B)(C \Omega+D)^{-1} \text {. }
$$

The map

induced by the map

$$
\varphi: \mathbb{C}^{g} / L_{\Omega_{*}}=\mathfrak{A}_{\Omega_{*}} \longrightarrow \mathfrak{A}_{\Omega}=\mathbb{C}^{g} / L_{\Omega}
$$

$$
\widetilde{\varphi}: \mathbb{C}^{g} \longrightarrow \mathbb{C}^{g}, \quad Z \longmapsto{ }^{t}(C \Omega+D) Z
$$

is a real isomorphism. Since $\widetilde{\varphi} \circ \sigma_{*}=\sigma \circ \widetilde{\varphi}$, i.e., $\widetilde{\varphi}$ commutes with complex conjugation on $\mathbb{C}^{g}$, we have $C=0$. Therefore

$$
\Omega_{*}=(A \Omega+B)^{t} A=\left(A X^{t} A+B^{t} A\right)+i A Y^{t} A,
$$

where $\Omega=X+i Y$. Hence we obtain the desired results (3.14) and (3.15).

Conversely we assume that there exists $A \in G L(g, \mathbb{Z})$ satisfying the conditions (3.14) and (3.15). Then

$$
\Omega_{*}=\gamma \cdot \Omega=(A \Omega+B)^{t} A
$$

for some $\gamma=\left(\begin{array}{cc}A & B \\ 0 & { }^{t} A^{-1}\end{array}\right) \in \Gamma_{g}$ with $B \in \mathbb{Z}^{(g, g)}$ with $B^{t} A=A^{t} B$. The map $\psi: \mathfrak{A}_{\Omega} \longrightarrow \mathfrak{A}_{\Omega_{*}}$ induced by the map

$$
\widetilde{\psi}: \mathbb{C}^{g} \longrightarrow \mathbb{C}^{g}, \quad Z \longmapsto A^{-1} Z
$$

is a complex isomorphism commuting complex conjugation $\sigma$. Therefore $\psi$ is a real isomorphism of $(\mathfrak{A}, E, \sigma)$ onto $\left(\mathfrak{A}_{*}, E_{*}, \sigma_{*}\right)$.

According to Theorem 3.3, we are led to define the subgroup $\Gamma_{g}^{\star}$ of $\Gamma_{g}$ by

$$
\Gamma_{g}^{\star}:=\left\{\left(\begin{array}{cc}
A & B \\
0 & { }^{t} A^{-1}
\end{array}\right) \in \Gamma_{g} \mid B \in \mathbb{Z}^{(g, g)}, A^{t} B=B^{t} A\right\} .
$$

It is easily seen that $\Gamma_{g}^{\star}$ acts on $\mathscr{H}_{g}$ properly discontinuously by

$$
\gamma \cdot \Omega=A \Omega^{t} A+B^{t} A,
$$

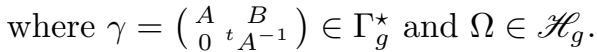




\section{Moduli spaces for real abelian varieties}

In Section 3 , we knew that $\Gamma_{g}^{\star}$ acts on $\mathscr{H}_{g}$ properly discontinuously by the formula (3.17). So the quotient space

$$
\mathscr{X}_{\mathbb{R}}^{g}:=\Gamma_{g}^{\star} \backslash \mathscr{H}_{g}
$$

inherits a structure of stratified real analytic space from the real analytic structure on $\mathscr{H}_{g}$. The stratified real analytic space $\mathscr{X}_{\mathbb{R}}^{g}$ classifies, up to real isomorphism, real principally polarized abelian varieties $(\mathfrak{A}, E, S)$ of dimension $g$. Thus $\mathscr{X}_{\mathbb{R}}^{g}$ is called the (real) moduli space of real principally polarized abelian varieties $(\mathfrak{A}, E, S)$ of dimension $g$. $[1]$

To study the structure of $\mathscr{X}_{\mathbb{R}}^{g}$, we need the following result of A. A. Albert

Lemma 4.1. Let $S_{g}(\mathbb{Z} / 2)$ be the set of all $g \times g$ symmetric matrices with coefficients in $\mathbb{Z} / 2$. We note that $G L(g, \mathbb{Z} / 2)$ acts on $S_{g}(\mathbb{Z} / 2)$ by $N \longmapsto A N^{t} A$ with $A \in G L(g, \mathbb{Z} / 2)$ and $N \in S_{g}(\mathbb{Z} / 2)$. We put

$$
\pi(N):=\prod_{k=1}^{g}\left(1-n_{k k}\right) \quad \text { for } N=\left(n_{i j}\right) \in S_{g}(\mathbb{Z} / 2) .
$$

Then $N \in S_{g}(\mathbb{Z} / 2)$ is equivalent $\bmod G L(g, \mathbb{Z} / 2)$ to a matrix of the form:

(I) $\left(\begin{array}{cc}I_{\lambda} & 0 \\ 0 & 0\end{array}\right)$ if $\pi(N)=0$ and $\operatorname{rank}(N)=\lambda$ or

(II) $\left(\begin{array}{cc}H_{\lambda} & 0 \\ 0 & 0\end{array}\right)$ with $H_{\lambda}:=\left(\begin{array}{ccc}0 & \cdots & 1 \\ \vdots & . \cdot & \vdots \\ 1 & \cdots & 0\end{array}\right) \in \mathbb{Z}^{(\lambda, \lambda)}$ if $\pi(N)=1$ and $\operatorname{rank}(N)$ $=\lambda$.

$N \in S_{g}(\mathbb{Z} / 2)$ is said to be diasymmetric in Case (I) and to be orthosymmetric in Case (II).

Theorem 4.1. Let $(\mathfrak{A}, E)$ be a principally polarized abelian variety of dimension $g$. Then there exists a real structure $S$ on $\mathfrak{A}$ such that $E$ is $S$-real if and only if $(\mathfrak{A}, E)$ admits a period matrix of the following form

$$
\left(I_{g}, \frac{1}{2} M+i Y\right), \quad Y \in \mathcal{P}_{g},
$$

where $M$ is one of the forms (I) and (II) in Lemma 4.1.

The above theorem is essentially due to Comessatti [6]. We refer to [24] or [25, Theorem 2.3, pp. 78-80 and Theorem 4.1, pp. 86-88] for the proof of the above theorem.

Lemma 4.2. Let $\Omega_{1}$ and $\Omega_{2}$ be two elements of $\mathscr{H}_{g}$ such that

$$
\Omega_{i}=\frac{1}{2} X_{i}+i Y_{i}, M_{i} \in \mathbb{Z}^{(g, g)}, Y_{i} \in \mathcal{P}_{g}, i=1,2 .
$$


Then $\Omega_{1}$ and $\Omega_{2}$ have images, under the natural projection $\pi_{g}: \mathscr{H}_{g} \longrightarrow \mathscr{X}_{\mathbb{R}}^{g}$, in the same connected component of $\mathscr{X}_{\mathbb{R}}^{g}$, if and only if $\operatorname{rank}\left(M_{1} \bmod 2\right)=$ $\operatorname{rank}\left(M_{2} \bmod 2\right)$ and $\pi\left(M_{1} \bmod 2\right)=\pi\left(M_{2} \bmod 2\right)$.

Theorem 4.2. $\mathscr{X}_{\mathbb{R}}^{g}$ is a real analytic manifold of dimension $g(g+1) / 2$ and has $g+1+\left[\frac{g}{2}\right]$ connected components. Moreover $\mathscr{X}_{\mathbb{R}}^{g}$ is semi-algebraic, i.e., $\mathscr{X}_{\mathbb{R}}^{g}$ is defined by a finite number of polynomial equalities and inequalities.

Proof. The proof can be found in [21, Theorem 6.1, p. 161].

Remark 4.1. Let $\Omega=\frac{1}{2} M+i Y \in \mathscr{H}_{g}$ with $M={ }^{t} M \in \mathbb{Z}^{(g, g)}$. If $\operatorname{rank}(M \bmod 2)=\lambda$,

then $\mathfrak{A}_{\Omega}(\mathbb{R})$ has $2^{g-\lambda}$ connected components (cf. [21, 24]). The other invariant $\pi\left(M_{2} \bmod 2\right)$ is an invariant related to the polarization.

Recall that by Lemma 4.1, the connected components of $\mathscr{X}_{\mathbb{R}}^{g}$ correspond to the different possible values of $(\lambda, i)=(\operatorname{rank}(M \bmod 2), \pi(M \bmod 2))$ on which we have the restriction:

$$
0 \leq \lambda \leq g, \quad i=0 \text { or } 1, \text { and } i=0 \text { if } \lambda \text { is odd, } \quad i=1 \text { if } \lambda=0 .
$$

We denote by $\mathscr{X}_{(\lambda, i)}^{g}$ the connected components of $\mathscr{X}_{\mathbb{R}}^{g}$ corresponding to the invariants $(\lambda, i)$.

Definition. Let $M \in \mathbb{Z}^{(g, g)}$ be a $g \times g$ symmetric integral matrix. We say that $M$ is of the standard form if $M$ is of one of the forms in Lemma 4.1 (we observe that for fixed $(\lambda, i)$ this form is unique).

Now we can prove the following.

Lemma 4.3. Let $M \in \mathbb{Z}^{(g, g)}$ be a symmetric integral matrix which is of the standard form with invariants $(\lambda, i)$. Let

$$
\Gamma_{(\lambda, i)}^{g}:=\left\{A \in G L(g, \mathbb{Z}) \mid A M^{t} A \equiv M(\bmod 2)\right\} .
$$

Then

$$
\mathscr{X}_{(\lambda, i)}^{g} \cong \Gamma_{(\lambda, i)}^{g} \backslash \mathcal{P}_{g}
$$

Proof. Let $[\Omega]$ be a class in $\mathscr{X}_{(\lambda, i)}^{g}$. By Lemma 4.1 and Lemma 4.2, there exist a symmetric integral matrix $M \in \mathbb{Z}^{(g, g)}$ with invariants $(\lambda, i)$ of the standard form and an element $Y \in \mathcal{P}_{g}$ such that $\frac{1}{2} M+i Y$ is a representative for the class $[\Omega]$. If $Y_{*} \in \mathcal{P}_{g}$ is such that $\frac{1}{2} M+i Y_{*}$ is also a representative for the class $[\Omega]$, according to Theorem 3.2 ,

$$
M \equiv A M^{t} A(\bmod 2) \text { and } Y_{*}=A Y^{t} A
$$

for some $A \in G L(g, \mathbb{Z})$.

Theorem 4.3. $\mathscr{X}_{(\lambda, i)}^{g}$ is a connected semi-algebraic set with a real analytic structure. 
Proof. The proof can be found in [21, p. 160].

Let $(\mathfrak{A}, E, S)$ be a real polarized abelian variety and $-S$ be the real structure obtained by composing $S$ with the involution $z \longmapsto-z$ of $\mathfrak{A}$. We see that $(\mathfrak{A}, E,-S)$ is also a real polarized abelian variety. In general $(\mathfrak{A}, E,-S)$ is not real isomorphic to $(\mathfrak{A}, E, S)$. Therefore the following correspondence

$$
\Sigma: \mathscr{X}_{\mathbb{R}}^{g} \longrightarrow \mathscr{X}_{\mathbb{R}}^{g}, \quad(\mathfrak{A}, E, S) \longmapsto(\mathfrak{A}, E,-S)
$$

defines a non-trivial involution of $\mathscr{X}_{\mathbb{R}}^{g}$.

Let $M \in \mathbb{Z}^{(g, g)}$ be a symmetric integral matrix which is of the standard form with invariants $(\lambda, i)$. It is easily checked that $M^{3}=M$. We put

$$
\Sigma_{M}:=\left(\begin{array}{cc}
-M & I_{g} \\
-\left(I_{g}+M^{2}\right) & M
\end{array}\right) .
$$

It is easy to see the following facts (4.4) and (4.5).

$$
\begin{gathered}
\Sigma_{M} \in \Gamma_{g} \text { and }\left(\Sigma_{M}\right)^{-1}=-\Sigma_{M} . \\
\left({ }^{t} \Sigma_{M}\right)^{-1}\left(\begin{array}{cc}
-I_{g} & 0 \\
M & I_{g}
\end{array}{ }^{t} \Sigma_{M}=\left(\begin{array}{cc}
I_{g} & 0 \\
-M & -I_{g}
\end{array}\right) .\right.
\end{gathered}
$$

Now we assume that $\Omega=\frac{1}{2} M+i Y \in \mathscr{H}_{g}$ represents $(\mathfrak{A}, E, S)$. By (3.13), the matrices of $S$ and $-S$ are given by

$$
M_{S}=\left(\begin{array}{cc}
-I_{g} & 0 \\
M & I_{g}
\end{array}\right) \quad \text { and } \quad M_{-S}=\left(\begin{array}{cc}
I_{g} & 0 \\
-M & -I_{g}
\end{array}\right)
$$

respectively with respect to the $\mathbb{R}$-basis given by the columns of $\left(\Omega, I_{g}\right)$. By the formulas (4.5) and (4.6) we see that $\Sigma_{M}(\Omega)$ represents the real polarized abelian variety.

Lemma 4.4. Let $M \in \mathbb{Z}^{(g, g)}$ be a symmetric integral matrix which is of the standard form with invariants $(\lambda, i)$ and $Y \in \mathcal{P}_{g}$. Then we have

$$
\Sigma_{M}\left(\frac{1}{2} M+i Y\right)=\frac{1}{2} M+i\left(\begin{array}{cc}
\frac{1}{2} I_{\lambda} & 0 \\
0 & I_{g-\lambda}
\end{array}\right) Y^{-1}\left(\begin{array}{cc}
\frac{1}{2} I_{\lambda} & 0 \\
0 & I_{g-\lambda}
\end{array}\right)^{-1}
$$

Proof. Using the fact that $M^{3}=M$, by a direct computation, we get

(4.8) $\Sigma_{M}\left(\frac{1}{2} M+i Y\right)=M\left(I_{g}+M^{2}\right)^{-1}+i\left(I_{g}-\frac{1}{2} M^{2}\right) Y^{-1}\left(I_{g}+M^{2}\right)^{-1}$.

It is easily checked that

$$
\left(I_{g}+M^{2}\right)^{-1}=I_{g}-\frac{1}{2} M^{2}=\left(\begin{array}{cc}
\frac{1}{2} I_{\lambda} & 0 \\
0 & I_{g-\lambda}
\end{array}\right) .
$$

The formula (4.7) follows immediately from (4.8) and (4.9). 
Proposition 4.1. The map $\Sigma: \mathscr{X}_{\mathbb{R}}^{g} \longrightarrow \mathscr{X}_{\mathbb{R}}^{g}$ defined by

$$
\Sigma([(\mathfrak{A}, E, S)]):=[(\mathfrak{A}, E,-S)], \quad[(\mathfrak{A}, E, S)] \in \mathscr{X}_{\mathbb{R}}^{g}
$$

is a real analytic involution of $\mathscr{X}_{\mathbb{R}}^{g}$. For each connected component $\mathscr{X}_{(\lambda, i)}^{g}$, we have

$$
\Sigma\left(\mathscr{X}_{(\lambda, i)}^{g}\right)=\mathscr{X}_{(\lambda, i)}^{g} .
$$

Hence $\Sigma$ leaves the connected components of $\mathscr{X}_{\mathbb{R}}^{g}$ globally fixed.

Proof. Let $M \in \mathbb{Z}^{(g, g)}$ be a symmetric integral matrix which is of the standard form with invariants $(\lambda, i)$. We denote by $\mathscr{H}_{g}(M)$ the connected component of $\mathscr{H}_{g}$ containing the matrices of the form $\frac{1}{2} M+i Y \in \mathscr{H}_{g}$ with $Y \in \mathcal{P}_{g}$. According to (4.5) and Lemma 4.4, we see that $\Sigma_{M}$ defines an involution of $\mathscr{H}_{g}(M)$. Since $\mathscr{H}_{g}(M)$ is mapped onto $\mathscr{X}_{(\lambda, i)}^{g}$, we obtain the desired result.

\section{Compactifications of the moduli space $\mathscr{X}_{\mathbb{R}}^{g}$}

In this section we review the compactification $\overline{\mathscr{X}_{\mathbb{R}}^{g}}$ of $\mathscr{X}_{\mathbb{R}}^{g}$ obtained by $\mathrm{R}$. Silhol [26] and the Baily-Borel compactification of $\Gamma_{g}(4 m) \backslash \mathbb{H}_{g}$ which is related to the moduli space of real abelian varieties with level $4 m$ structure.

First of all we recall the Satake compactification of the Siegel modular variety $\mathcal{A}_{g}:=\Gamma_{g} \backslash \mathbb{H}_{g}$. Let

$$
\mathbb{D}_{g}:=\left\{W \in \mathbb{C}^{(g, g)} \mid W={ }^{t} W, I_{g}-\bar{W} W>0\right\}
$$

be the generalized unit disk of degree $g$ which is a bounded realization of $\mathbb{H}_{g}$. In fact, the Cayley transform $\Phi_{g}: \mathbb{D}_{g} \longrightarrow \mathbb{H}_{g}$ defined by

$$
\Phi_{g}(W):=i\left(I_{g}+W\right)\left(I_{g}-W\right)^{-1}, \quad W \in \mathbb{D}_{g}
$$

is a biholomorphic mapping of $\mathbb{D}_{g}$ onto $\mathbb{H}_{g}$ which gives the bounded realization of $\mathbb{H}_{g}$ by $\mathbb{D}_{g}$ [23, pp. 281-283]. The inverse $\Psi_{g}$ of $\Phi_{g}$ is given by

$$
\Psi_{g}(\Omega)=\left(\Omega-i I_{g}\right)\left(\Omega+i I_{g}\right)^{-1}, \quad \Omega \in \mathbb{H}_{g} .
$$

We let

$$
T=\frac{1}{\sqrt{2}}\left(\begin{array}{cc}
I_{g} & I_{g} \\
i I_{g} & -i I_{g}
\end{array}\right)
$$

be the $2 g \times 2 g$ matrix represented by $\Phi_{g}$. Then

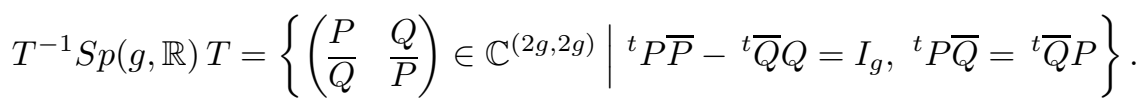

Indeed, if $M=\left(\begin{array}{cc}A & B \\ C & D\end{array}\right) \in S p(g, \mathbb{R})$, then

$$
T^{-1} M T=\left(\begin{array}{ll}
\frac{P}{Q} & \frac{Q}{P}
\end{array}\right)
$$

where

$$
P=\frac{1}{2}\{(A+D)+i(B-C)\}
$$


and

$$
Q=\frac{1}{2}\{(A-D)-i(B+C)\}
$$

For brevity, we set

$$
G_{*}=T^{-1} S p(g, \mathbb{R}) T .
$$

Then $G_{*}$ is a subgroup of $S U(g, g)$, where

$$
S U(g, g)=\left\{h \in \mathbb{C}^{(2 g, 2 g)} \mid{ }^{t} h I_{g, g} \bar{h}=I_{g, g}, \operatorname{det} h=1\right\}, \quad I_{g, g}=\left(\begin{array}{cc}
I_{g} & 0 \\
0 & -I_{g}
\end{array}\right) .
$$

In the case $g=1$, we observe that

$$
T^{-1} S p(1, \mathbb{R}) T=T^{-1} S L_{2}(\mathbb{R}) T=S U(1,1) .
$$

If $g>1$, then $G_{*}$ is a proper subgroup of $S U(g, g)$. In fact, since ${ }^{t} T J_{g} T=-i J_{g}$, we get

$$
G_{*}=\left\{h \in S U(g, g) \mid{ }^{t} h J_{g} h=J_{g}\right\} .
$$

Let

$$
P^{+}=\left\{\left(\begin{array}{cc}
I_{g} & Z \\
0 & I_{g}
\end{array}\right) \mid Z={ }^{t} Z \in \mathbb{C}^{(g, g)}\right\}
$$

be the $P^{+}$-part of the complexification of $G_{*} \subset S U(g, g)$.

Since the Harish-Chandra decomposition of an element $\left(\frac{P}{Q} \frac{Q}{P}\right)$ in $G_{*}^{J}$ is

$$
\left(\begin{array}{ll}
P & \bar{Q} \\
\bar{P}
\end{array}\right)=\left(\begin{array}{cc}
I_{g} & Q \bar{P}^{-1} \\
0 & I_{g}
\end{array}\right)\left(\begin{array}{cc}
P-Q \bar{P}^{-1} \bar{Q} & 0 \\
0 & \bar{P}
\end{array}\right)\left(\begin{array}{cc}
I_{g} & 0 \\
\bar{P}^{-1} \bar{Q} & I_{g}
\end{array}\right),
$$

the $P^{+}$-component of the following element

$$
\left(\begin{array}{cc}
P & Q \\
\bar{P}
\end{array}\right) \cdot\left(\begin{array}{cc}
I_{g} & W \\
0 & I_{g}
\end{array}\right), \quad W \in \mathbb{D}_{g}
$$

of the complexification of $G_{*}^{J}$ is given by

$$
\left(\left(\begin{array}{cc}
I_{g} & (P W+Q)(\bar{Q} W+\bar{P})^{-1} \\
0 & I_{g}
\end{array}\right)\right) .
$$

We note that $Q \bar{P}^{-1} \in \mathbb{D}_{g}$. We get the Harish-Chandra embedding of $\mathbb{D}_{g}$ into $P^{+}$(cf. $\left[12\right.$, p. 155] or $\left[19\right.$, pp. 58-59]). Therefore we see that $G_{*}$ acts on $\mathbb{D}_{g}$ transitively by

$$
\left(\begin{array}{ll}
\frac{P}{Q} & \frac{Q}{P}
\end{array}\right) \cdot W=(P W+Q)(\bar{Q} W+\bar{P})^{-1}, \quad\left(\begin{array}{ll}
\frac{P}{Q} & \frac{Q}{P}
\end{array}\right) \in G_{*}, W \in \mathbb{D}_{g}
$$

The isotropy subgroup at the origin $o$ is given by

$$
K=\left\{\left(\begin{array}{cc}
P & 0 \\
0 & P
\end{array}\right) \mid P \in U(g)\right\} .
$$

Thus $G_{*} / K$ is biholomorphic to $\mathbb{D}_{g}$. The action (2.4) is compatible with the action (5.6) via the Cayley transform (5.2). 
In summary, $S p(g, \mathbb{R})$ acts on $\mathbb{D}_{g}$ transitively by

$$
\left(\begin{array}{ll}
A & B \\
C & D
\end{array}\right) \cdot W=(P W+Q)(\bar{Q} W+\bar{P})^{-1}, \quad\left(\begin{array}{ll}
A & B \\
C & D
\end{array}\right) \in S p(g, \mathbb{R}), W \in \mathbb{D}_{g},
$$

where $P$ and $Q$ are given by (5.4) and (5.5). This action extends to the closure $\overline{\mathbb{D}}_{g}$ of $\mathbb{D}_{g}$ in $\mathbb{C}^{g(g+1) / 2}$.

For an integer $s$ with $0 \leq s \leq g$, we let

$$
\mathscr{F}_{s}:=\left\{W=\left(\begin{array}{cc}
W_{1} & 0 \\
0 & I_{g-s}
\end{array}\right) \mid W_{1} \in \mathbb{D}_{s}\right\} \subset \overline{\mathbb{D}}_{g} .
$$

We say that $\mathscr{F}_{s}$ is the standard boundary component of degree $s$. If there exists an element $\gamma \in S p(g, \mathbb{Q})$ (equivalently $\gamma \in \Gamma_{g}$ ) with $\mathscr{F}=\gamma\left(\mathscr{F}_{s}\right) \subset \overline{\mathbb{D}}_{g}$, then $\mathscr{F}$ is said to be a rational boundary component of degree $s$. The Siegel upper half plane $\mathbb{H}_{s}$ is attached to $\mathbb{H}_{g}$ as a limit of matrices in $\mathbb{C}^{(g, g)}$ by

$$
\Omega_{1} \longmapsto \lim _{Y \longrightarrow \infty}\left(\begin{array}{cc}
\Omega_{1} & 0 \\
0 & i Y
\end{array}\right), \quad \Omega_{1} \in \mathbb{H}_{s}, Y \in \mathcal{P}_{g-s},
$$

meaning that all the eigenvalues of $Y$ converge to $\infty$.

For a rational boundary component $\mathscr{F} \subset \overline{\mathbb{D}}_{g}$, we let

$$
P(\mathscr{F})=\{\alpha \in S p(g, \mathbb{Q}) \mid \alpha(\mathscr{F})=\mathscr{F}\}
$$

be the normalizer in $S p(g, \mathbb{Q})$ of $\mathscr{F}$ (or the parabolic subgroup of $S p(g, \mathbb{Q}$ ) associated to $\mathscr{F})$ and let

$$
Z(\mathscr{F})=\{\alpha \in S p(g, \mathbb{Q}) \mid \alpha(W)=W \text { for all } W \in \mathscr{F}\}
$$

be the centralizer of $\mathscr{F}$. We put

$$
G(\mathscr{F}):=P(\mathscr{F}) / Z(\mathscr{F}) \cong S p(s, \mathbb{Q}) .
$$

Obviously $G(\mathscr{F})$ acts on $\mathscr{F}$. We choose the standard boundary component $\mathscr{F}=\mathscr{F}_{s}$. An element $\gamma$ of $P(\mathscr{F})$ is of the form

$$
\gamma=\left(\begin{array}{cccc}
A_{1} & 0 & B_{1} & * \\
* & u & * & * \\
C_{1} & 0 & D_{1} & * \\
0 & 0 & 0 & { }^{t} u^{-1}
\end{array}\right) \in S p(g, \mathbb{Q})
$$

where

$$
\gamma_{1}=\left(\begin{array}{ll}
A_{1} & B_{1} \\
C_{1} & D_{1}
\end{array}\right) \in S p(s, \mathbb{Q}) \quad \text { and } \quad u \in G L(g-s, \mathbb{Q}) .
$$

The unipotent radical $U(\mathscr{F})$ of $P(\mathscr{F})$ is given by

$$
U(\mathscr{F})=\left\{\left(\begin{array}{cccc}
I_{s} & 0 & 0 & { }^{t} \mu \\
\lambda & I_{g-s} & \mu & \kappa \\
0 & 0 & I_{s} & -{ }^{t} \lambda \\
0 & 0 & 0 & I_{g-s}
\end{array}\right) \mid \lambda, \mu \in \mathbb{Q}^{(g-s, s)}, \kappa \in \mathbb{Q}^{(g-s, g-s)}\right\}
$$


and the centralizer $Z_{U}(\mathscr{F})$ of $U(\mathscr{F})$ is given by

$$
Z_{U}(\mathscr{F})=\left\{\left(\begin{array}{cccc}
I_{s} & 0 & 0 & 0 \\
0 & I_{g-s} & 0 & \kappa \\
0 & 0 & I_{s} & 0 \\
0 & 0 & 0 & I_{g-s}
\end{array}\right) \mid \kappa \in \mathbb{Q}^{(g-s, g-s)}\right\} .
$$

We have inclusions of normal subgroups

$$
Z_{U}(\mathscr{F}) \subset U(\mathscr{F}) \subset P(\mathscr{F}) .
$$

The Levi factor $L(\mathscr{F})$ of $P(\mathscr{F})$ is given by

$$
L(\mathscr{F})=G_{h}(\mathscr{F}) G_{l}(\mathscr{F})
$$

$$
G_{h}(\mathscr{F})=\left\{\left(\begin{array}{cccc}
A_{1} & 0 & B_{1} & 0 \\
0 & I_{g-s} & 0 & 0 \\
C_{1} & 0 & D_{1} & 0 \\
0 & 0 & 0 & I_{g-s}
\end{array}\right) \in P(\mathscr{F}) \mid\left(\begin{array}{cc}
A_{1} & B_{1} \\
C_{1} & D_{1}
\end{array}\right) \in \operatorname{Sp}(s, \mathbb{Q})\right\}
$$

and

$$
G_{l}(\mathscr{F})=\left\{\left(\begin{array}{cccc}
I_{s} & 0 & 0 & 0 \\
0 & S & 0 & 0 \\
0 & 0 & I_{s} & 0 \\
0 & 0 & 0 & { }^{t} S^{-1}
\end{array}\right) \in P(\mathscr{F}) \mid S \in G L(g-s, \mathbb{Q})\right\} .
$$

The subgroup $U(\mathscr{F}) G_{h}(\mathscr{F})$ is normal in $P(\mathscr{F})$. The map $P(\mathscr{F}) \longrightarrow S p(s, \mathbb{Q})$, $\gamma \mapsto \gamma_{1}$ is surjective and induces the isomorphism $G_{h}\left(\mathscr{F}_{s}\right) \cong S p(s, \mathbb{Q})$. We note that the map $f: P\left(\mathscr{F}_{s}\right) \cap S p(g, \mathbb{Z}) \longrightarrow S p(s, \mathbb{Z}), \gamma \mapsto \gamma_{1}$ is obtained via $\left(\begin{array}{cc}W & 0 \\ 0 & I_{g-s}\end{array}\right) \longmapsto W$, in the sense that if $\gamma \in P\left(\mathscr{F}_{s}\right)$, then

$$
\gamma \cdot\left(\begin{array}{cc}
W & 0 \\
0 & I_{g-s}
\end{array}\right)=\left(\begin{array}{cc}
\gamma_{1}(W) & 0 \\
0 & I_{g-s}
\end{array}\right)
$$

We define

$$
\mathbb{D}_{g}^{s t}:=\coprod_{0 \leq s \leq g} \mathscr{F}_{s}
$$

and

$$
\mathbb{D}_{g}^{*}:=\coprod_{\mathscr{F} \text { :rational }} \mathscr{F},
$$

where $\mathscr{F}$ runs over all rational boundary components. Via the Cayley transform $\Phi_{g}$ (cf. (5.2)), we identify

$$
\mathbb{D}_{g}^{s t}=\mathbb{H}_{g}^{s t}=\coprod_{0 \leq s \leq g} \mathbb{H}_{s}
$$


Definition. Let $u>1$. We denote by $\mathfrak{W}_{g}(u)$ the set of all matrices $\Omega=X+i Y$ in $\mathbb{H}_{g}$ with $X=\left(x_{i j}\right) \in \mathbb{R}^{(g, g)}$ satisfying the conditions $(\Omega 1)$ and $(\Omega 2)$ :

$(\Omega 1)\left|x_{i j}\right|<u$;

$(\Omega 2)$ if $Y={ }^{t} W D W$ is the Jacobi decomposition of $Y$ with $W=\left(w_{i j}\right)$ strictly upper triangular and $D=\operatorname{diag}\left(d_{1}, \ldots, d_{g}\right)$ diagonal, then we have

$$
\left|w_{i j}\right|<u, \quad 1<u d_{1}, \quad d_{i}<u d_{i+1}, \quad i=1, \ldots, g-1 .
$$

It is well known that for sufficiently large $u>0$, the set $\mathfrak{W}_{g}(u)$ is a fundamental set for the action of $\Gamma_{g}$ on $\mathbb{H}_{g}$, that is, $\Gamma_{g} \cdot \mathfrak{W}_{g}(u)=\mathbb{H}_{g}$, and

$$
\left\{\gamma \in \Gamma_{g} \mid \gamma \cdot \mathfrak{W}_{g}(u) \cap \mathfrak{W}_{g}(u) \neq \emptyset\right\}
$$

is a finite set. We observe that if $\Omega=\left(\begin{array}{cc}\Omega_{1} & \Omega_{3} \\ { }^{{ }_{3}} \Omega_{3} & \Omega_{2}\end{array}\right) \in \mathfrak{W}_{g}(u)$ with $\Omega_{1} \in \mathbb{C}^{(s, s)}$, then $\Omega_{1} \in \mathfrak{W}_{s}(u)$.

Definition. We can choose a sufficiently large $u_{0}>0$ such that for all $0 \leq$ $s \leq g, \mathfrak{W}_{s}\left(u_{0}\right)$ is a fundamental set for the action of $\Gamma_{s}$ on $\mathbb{H}_{s}$. In this case we simply write $\mathfrak{W}_{s}=\mathfrak{W}_{s}\left(u_{0}\right)$ with $0 \leq s \leq g$. We define

$$
\mathfrak{W}_{g}^{*}:=\coprod_{0 \leq s \leq g} \mathfrak{W}_{s} .
$$

For $\Omega_{*} \in \mathfrak{W}_{g-r}$, we let $U$ be a neighborhood of $\Omega_{*}$ in $\mathfrak{W}_{g-r}$ and $v$ a positive real number. For $0 \leq s \leq r$, we let $W_{s}(U, v)$ be the set of all

$$
\Omega=\left(\begin{array}{cc}
\Omega_{1} & \Omega_{3} \\
{ }^{t} \Omega_{3} & \Omega_{2}
\end{array}\right) \in \mathfrak{W}_{g-s} \quad \text { with } \quad \Omega_{1} \in \mathbb{C}^{(g-r, g-r)}
$$

satisfying the conditions ( $\mathfrak{W} 1)$ and $(\mathfrak{W} 1)$ :

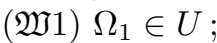

(W्W2) if $Y={ }^{t} W D W$ is the Jacobi decomposition of $Y$ with $W$ strictly upper triangular and $D=\operatorname{diag}\left(d_{1}, \ldots, d_{g}\right)$ diagonal, then we have $d_{g-r+1}>v$.

A fundamental set of neighborhoods of $\Omega_{*} \in \mathfrak{W}_{g-r}$ for the Satake topology on $\mathfrak{W}_{g}^{*}$ is given by the collection $\left\{\bigcup_{0 \leq s \leq r} W_{s}(U, v)\right\}$ 's, where $U$ runs through neighborhoods of $\Omega_{*}$ in $\mathfrak{W}_{g-r}$ and $v$ ranges in $\mathbb{R}^{+}$. We regard

as a subset of $\mathbb{D}_{g}^{*}$.

$$
\mathfrak{W}_{g}^{*} \subset \mathbb{H}_{g}^{s t} \cong \mathbb{D}_{g}^{s t}
$$

The Satake topology on $\mathbb{D}_{g}^{*}$ is characterized as the unique topology $\mathscr{T}$ extending the ordinary matrix topology on $\mathbb{D}_{g}$ and satisfying the following properties (ST1)-(ST4):

(ST1) $\mathscr{T}$ induces on $\mathfrak{W}_{g}^{*}$ the topology defined in Definition 5.2;

$(\mathrm{ST} 2) S p(g, \mathbb{Q})$ acts continuously on $\mathbb{D}_{g}^{*}$;

(ST3) $\mathcal{A}_{g}^{*}=\Gamma_{g} \backslash \mathbb{D}_{g}^{*}$ is a compact Hausdorff space;

(ST4) For any $\Omega \in \mathbb{D}_{g}^{*}$, there exists a fundamental set of neighborhoods $\{U\}$ of $\Omega$ such that $\gamma \cdot U=U$ if $\gamma \in \Gamma_{g}(\Omega):=\left\{\gamma \in \Gamma_{g} \mid \gamma \cdot \Omega=\Omega\right\}$, and $\gamma \cdot U \cap U=\emptyset$ if $\gamma \notin \Gamma_{g}(\Omega)$. 
For a proof of these above facts we refer to [4].

Now we are ready to investigate the compactification of the moduli space $\mathscr{X}_{\mathbb{R}}^{g}$ of real principally polarized abelian varieties of dimension $g$ obtained by R. Silhol.

Definition. Let $u>1$. We let $F_{g}(u)$ be the set of all $\Omega=X+i Y \in \mathscr{H}_{g}$ with $X=\operatorname{Re} \Omega=\left(x_{i j}\right)$ satisfying the following conditions (a) and (b):

(a) $x_{i j}=0$ or $\frac{1}{2}$;

(b) if $Y={ }^{t} W D W$ is the Jacobi decomposition of $Y$ with $W$ strictly upper triangular and $D=\operatorname{diag}\left(d_{1}, \ldots, d_{g}\right)$ diagonal, then we have

$$
\left|w_{i j}\right| \leq u \quad \text { and } \quad 0<d_{i} \leq u d_{i+1} .
$$

We define $F_{g}^{\prime}(u)$ to be the set of matrices in $\mathscr{H}_{g}$ satisfying the condition $\left|x_{i j}\right| \leq$ $\frac{1}{2}$ and the above condition (b). Let $u_{0}>$ be as in Definition 5.2. We put

$$
F_{g}:=F_{g}\left(u_{0}\right) \text {. }
$$

It is well known that $F_{g}$ is a fundamental set for the action of $\Gamma_{g}^{\star}$ on $\mathscr{H}_{g}$.

For two nonnegative integers $s$ and $t$, we define two subsets $\mathscr{F}_{s, t}$ and $F_{s, t}$ of $\mathbb{D}_{g}^{*}$ as follows.

$$
\mathscr{F}_{s, t}:=\left\{\left(\begin{array}{ccc}
-I_{s} & 0 & 0 \\
0 & W & 0 \\
0 & 0 & I_{t}
\end{array}\right) \in \mathbb{D}_{g}^{*} \mid W \in \mathbb{D}_{g-(s+t)}\right\}
$$

and

$$
F_{s, t}:=\left\{\left(\begin{array}{ccc}
-I_{s} & 0 & 0 \\
0 & W & 0 \\
0 & 0 & I_{t}
\end{array}\right) \in \mathscr{F}_{s, t} \mid W \in F_{g-(s+t)}\right\} .
$$

For $M \in \mathbb{Z}^{(g, g)}$, we set

$$
F_{M}:=\left\{\Omega \in F_{g} \mid 2 \operatorname{Re} \Omega=M\right\} .
$$

In particular, $F_{\mathbf{0}}=\left\{\Omega \in F_{g} \mid \operatorname{Re} \Omega=0\right\}$, where $\mathbf{0}$ denotes the $g \times g$ zero matrix. We let

$$
\mathcal{M}:=\left\{M=\left(m_{i j}\right) \in \mathbb{Z}^{(g, g)} \mid M={ }^{t} M, m_{i j}=0 \text { or } 1\right\} .
$$

For any $M \in \mathcal{M}$, we set

$$
B_{M}:=\left(\begin{array}{cc}
I_{g} & \frac{1}{2} M \\
0 & I_{g}
\end{array}\right) \in S p(g, \mathbb{Q}) .
$$

By the definition we have

$$
F_{g}=\bigcup_{M \in \mathcal{M}} B_{M}\left(F_{\mathbf{0}}\right) \text { and } \bar{F}_{g}=\bigcup_{M \in \mathcal{M}} B_{M}\left(\bar{F}_{\mathbf{0}}\right) .
$$

We can show that $\overline{\mathscr{H}_{g}}=\Gamma_{g}^{\star} \cdot \bar{F}_{g}$. 
Now we embed $\mathscr{H}_{g}$ into $\mathbb{D}_{g}^{*}$ via the Cayley transform (5.3). We let $\overline{\mathscr{H}_{g}}$ be the closure of $\mathscr{H}_{g}$ in $\mathbb{D}_{g}^{*}$. Then the action of $\Gamma_{g}^{\star}$ extends to an action of $\Gamma_{g}^{\star}$ on $\overline{\mathscr{H}_{g}}$ (see (3.16), (3.17), (ST2)). R. Silhol proved that the quotient space

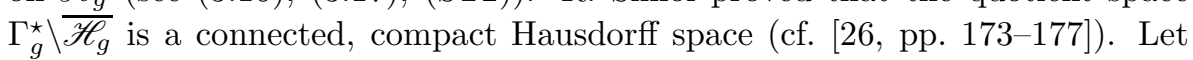
$\pi: \overline{\mathscr{H}}_{g} \longrightarrow \Gamma_{g}^{\star} \backslash \overline{\mathscr{H}}_{g}$ be the canonical projection. For $M \in \mathcal{M}$, we define

$$
\mathscr{H}_{M}=\left\{\frac{1}{2} M+i Y \in \mathscr{H}_{g}\right\} .
$$

We let $\overline{\mathscr{H}}_{M}$ be the closure of $\mathscr{H}_{M}$ in $\overline{\mathscr{H}}_{g}$. Then without difficulty we can see that

$$
\Gamma_{g}^{\star} \backslash \overline{\mathscr{H}_{g}}=\bigcup_{0 \leq s+t \leq g} \bigcup_{M \in \mathcal{M}}\left(\pi\left(B_{M}\left(\mathscr{F}_{s, t}\right) \cup \overline{\mathscr{H}}_{\mathbf{0}}\right)\right) .
$$

Let $\left\{\mathscr{X}_{i} \mid 1 \leq i \leq N\right\}$ with $N=g+1+\left[\frac{g}{2}\right]$ be the connected components of $\mathscr{X}_{\mathbb{R}}^{g} \subset \Gamma_{g}^{\star} \backslash \overline{\mathscr{H}_{g}}$ and let $\Sigma_{i}$ be the restriction to $\mathscr{X}_{i}$ of the fundamental involution $\Sigma$ (cf. Proposition 4.1). We note that $\Sigma$ does not extend to a global involution of $\Gamma_{g}^{\star} \backslash \frac{\mathscr{H}_{g}}{}$. But $\Sigma_{i}$ extends to an involution of the closure $\overline{\mathscr{X}}_{i}$ of $\mathscr{X}_{i}$ in $\Gamma_{g}^{\star} \backslash \overline{\mathscr{H}}_{g}$. We observe that for each $1 \leq i \leq N$, we have $\overline{\mathscr{X}}_{i}=\Gamma_{g}^{\star}\left(M_{i}\right) \backslash \overline{\mathscr{H}}_{M_{i}}$ for some $M_{i} \in \mathcal{M}$. Here $\Gamma_{g}^{\star}\left(M_{i}\right)=\left\{\gamma \in \Gamma_{g}^{\star} \mid \gamma\left(\mathscr{H}_{M_{i}}\right)=\mathscr{H}_{M_{i}}\right\}$.

Definition. Let $z_{1} \in \overline{\mathscr{X}}_{i}$ and $z_{2} \in \overline{\mathscr{X}}_{j}$. We say that $z_{1}$ and $z_{2}$ are $\Sigma$-equivalent and write $z_{1} \sim z_{2}$ if $\Sigma_{i}\left(z_{1}\right)=\Sigma_{j}\left(z_{2}\right)$.

Silhol $\left[26\right.$, p. 185] showed that $\sim$ defines an equivalence relation in $\Gamma_{g}^{\star} \backslash \overline{\mathscr{H}_{g}}$.

By a direct computation, we obtain

$$
P\left(\mathscr{F}_{s, t}\right)=\left\{\left(\begin{array}{cccccc}
v_{1} & 0 & 0 & 0 & 0 & v_{21} \\
* & A_{1} & 0 & 0 & B_{1} & * \\
* & * & u_{2} & -u_{21} & * & * \\
* & * & -u_{12} & u_{1} & * & * \\
* & C_{1} & 0 & 0 & D_{1} & * \\
v_{12} & 0 & 0 & 0 & 0 & v_{2}
\end{array}\right) \in S p(g, \mathbb{Q})\right\}
$$

where

$$
\gamma_{1}=\left(\begin{array}{ll}
A_{1} & B_{1} \\
C_{1} & D_{1}
\end{array}\right) \in S p(g-r, \mathbb{Q}) \quad \text { with } \quad r=s+t
$$

and

$$
U=\left(\begin{array}{cc}
u_{1} & u_{12} \\
u_{21} & u_{2}
\end{array}\right) \in G L(r, \mathbb{Q}), \quad V=\left(\begin{array}{cc}
v_{1} & v_{21} \\
v_{12} & v_{2}
\end{array}\right)={ }^{t} U^{-1} .
$$

Now we define

$$
\mathscr{X}_{\mathbb{R}}^{g}(s, t):=\left(\Gamma_{g}^{\star} \cap P\left(\mathscr{F}_{s, t}\right)\right) \backslash\left(\mathscr{F}_{s, t} \cap \overline{\mathscr{H}_{g}}\right) .
$$

It is easily checked that

$$
\Gamma_{g}^{\star} \cap P\left(\mathscr{F}_{s, t}\right) \cong \Gamma_{g-(s+t)}^{\star} \quad \text { and } \quad \mathscr{F}_{s, t} \cap \overline{\mathscr{H}}_{g} \cong \overline{\mathscr{H}}_{g-(s+t)} .
$$


We define

$$
\overline{\mathscr{X}_{\mathbb{R}}^{g}}:=\Gamma_{g}^{\star} \backslash \overline{\mathscr{H}_{g}} / \sim .
$$

Silhol [26, Theorem 8.17] proved the following theorem.

Theorem 5.1. $\overline{\mathscr{X}_{\mathbb{R}}^{g}}$ is a connected compact Hausdorff space containing $\mathscr{X}_{\mathbb{R}}^{g}$ as a dense open subset. As a set,

$$
\overline{\mathscr{X}_{\mathbb{R}}^{g}}=\coprod_{0 \leq s+t \leq g} \mathscr{X}_{\mathbb{R}}^{g}(s, t) .
$$

We recall that $\mathbb{H}_{g}^{*}$ denotes the Satake partial compactification of $\mathbb{H}_{g}$ that is obtained by attaching all rational boundary components with the Satake topology. We know that $S p(g, \mathbb{Q})$ acts on $\mathbb{H}_{g}^{*}$, the involution $\tau: \mathbb{H}_{g} \longrightarrow \mathbb{H}_{g}$ (cf. $(2.9))$ extends to $\mathbb{H}_{g}^{*}$ and $\tau(\alpha \cdot x)=\tau(\alpha) \tau(x)$ for all $\alpha \in S p(g, \mathbb{Q})$ and $x \in \mathbb{H}_{g}^{*}$.

Let $N=4 m$ with $m$ a positive integer. We write

$$
X(N):=\Gamma_{g}(N) \backslash \mathbb{H}_{g} \quad \text { and } \quad V(N):=\Gamma_{g}(N) \backslash \mathbb{H}_{g}^{*} .
$$

We let

$$
\pi_{B B}: \mathbb{H}_{g}^{*} \longrightarrow V(N)=\Gamma_{g}(N) \backslash \mathbb{H}_{g}^{*}
$$

be the canonical projection of $\mathbb{H}_{g}^{*}$ to the Baily-Borel compactification of $X(N)$. The involution $\tau$ passes to complex conjugation $\tau: V(N) \longrightarrow V(N)$, whose fixed points we denote by $V(N)_{\mathbb{R}}$. Obviously the $\tau$-fixed set

$$
X(N)_{\mathbb{R}}:=\{x \in X(N) \mid \tau(x)=x\}
$$

is a subset of $V(N)_{\mathbb{R}}$. We let $\overline{X(N)}_{\mathbb{R}}$ denote the closure of $X(N)_{\mathbb{R}}$ in $V(N)_{\mathbb{R}}$.

Theorem 5.2. There exists a natural rational structure on $V(N)$ which is compatible with the real structure defined by $\tau$.

Proof. It follows from Shimura's result [22] that the $\Gamma_{g}(N)$-automorphic forms on $\mathbb{H}_{g}$ are generated by those automorphic forms with rational Fourier coefficients.

If $\gamma \in \Gamma_{g}(N)$ and $\mathscr{F}$ is a rational boundary component of $\mathbb{H}_{g}^{*}$ such that $\tau(\mathscr{F})=\mathscr{F}$, we define the set of $\gamma$-real points of $\mathscr{F}$ to be

$$
\mathscr{F}^{\tau \gamma}:=\{x \in \mathscr{F} \mid \tau(x)=\gamma \cdot x\} .
$$

Then $\pi_{B B}\left(\mathscr{F}^{\tau \gamma}\right) \subset V(N)_{\mathbb{R}}$.

Definition. Let $N=4 m$. A $\Gamma_{g}(N)$-real boundary pair $(\mathscr{F}, \gamma)$ of degree $s$ consists of a rational boundary component $\mathscr{F}$ of degree $s$ and an element $\gamma \in \Gamma_{g}(N)$ such that $\mathscr{F}^{\tau \gamma} \neq \emptyset$. We say that two $\Gamma_{g}(N)$-real boundary components $(\mathscr{F}, \gamma)$ and $\left(\mathscr{F}_{*}, \gamma_{*}\right)$ are equivalent if the resulting loci of real points $\pi_{B B}\left(\mathscr{F}^{\tau \gamma}\right)=\pi_{B B}\left(\mathscr{F}_{*}^{\tau \gamma}\right)$ coincide. 
We observe that if $(\mathscr{F}, \gamma)$ is a $\Gamma_{g}(N)$-real boundary pair and if $\alpha \in \Gamma_{g}(N)$, we see that $\tau(\mathscr{F})=\gamma(\mathscr{F})$ and $\left(\alpha(\mathscr{F}), \tau(\alpha) \gamma \alpha^{-1}\right)$ is an equivalent $\Gamma_{g}(N)$-real boundary pair.

Fix a positive integer $s$ with $1 \leq s \leq g$. We define the map $\Phi: \mathbb{H}_{s} \longrightarrow \mathbb{H}_{g}^{*}$ by

$$
\Phi\left(\Omega_{1}\right)=\lim _{Y \longrightarrow \infty}\left(\begin{array}{cc}
\Omega_{1} & 0 \\
0 & i Y
\end{array}\right), \quad \Omega_{1} \in \mathbb{H}_{s}, Y \in \mathcal{P}_{g-s} .
$$

Obviously $\Phi\left(\mathbb{H}_{s}\right)=\mathscr{F}_{s}$ is the standard boundary component of degree $s$ (cf. $(5.8))$.

Let

$$
\nu_{s}: P\left(\mathscr{F}_{s}\right) \longrightarrow G_{h}\left(\mathscr{F}_{s}\right)
$$

be the projection to the quotient. It is easily seen that $\nu_{s}$ commutes with $\tau$. Therefore $\mathscr{F}_{s}$ is preserved by $\tau$. The set

$$
\mathscr{F}_{s}^{\tau}=\left\{\Phi(i Y) \mid Y \in \mathcal{P}_{s}\right\}
$$

is the set of $\tau$-fixed points in $\mathscr{F}_{s}$ and may be canonically identified with $\mathcal{P}_{s}$. We denote by $i I_{s}$ its canonical base point. Then $\mathscr{F}_{s}$ is attached to $\mathbb{H}_{g}$ so that the cone $\Phi\left(i \mathcal{P}_{s}\right)$ is contained in the closure of the cone $i \mathcal{P}_{g}$.

Proposition 5.1. Let $(\mathscr{F}, \gamma)$ be a $\Gamma_{g}(N)$-real boundary pair of degree s. Then there exists $\gamma_{*} \in \Gamma_{g}$ such that $\gamma_{*}\left(\mathscr{F}_{s}\right)=\mathscr{F}$ and

$$
\tau\left(\gamma_{*}\right)^{-1} \gamma \gamma_{*}=\left(\begin{array}{cc}
A & B \\
0 & { }^{t} A^{-1}
\end{array}\right) \in \operatorname{ker}\left(\nu_{s}\right)
$$

Moreover, we may take $B=0$, i.e., there exist $\gamma^{\prime} \in \Gamma_{g}(4 m)$ and $\gamma_{0} \in \Gamma_{g}$ so that $\mathscr{F}^{\tau \gamma^{\prime}}=\mathscr{F}^{\tau \gamma}, \gamma_{0}\left(\mathscr{F}_{s}\right)=\mathscr{F}$, and so that

$$
\tau\left(\gamma_{0}\right)^{-1} \gamma^{\prime} \gamma_{0}=\left(\begin{array}{cc}
A & 0 \\
0 & { }^{t} A^{-1}
\end{array}\right) \in \operatorname{ker}\left(\nu_{s}\right) .
$$

Proof. The proof can be found in [8, pp. 19-21].

As an application of Proposition 5.1, we get the following theorem.

Theorem 5.3. Let $m \geq 1$ be a positive integer. Let $\mathscr{F}$ be a proper rational boundary component of $\mathbb{H}_{g}$ of degree $g-1$. Let $\gamma \in \Gamma_{g}(4 m)$ such that

$$
\mathscr{F}^{\tau \gamma}=\{x \in \mathscr{F} \mid \tau(x)=\gamma \cdot x\} \neq \emptyset .
$$

Then $\mathscr{F} \tau \gamma$ is contained in the closure of $\mathbb{H}_{g}^{\tau \Gamma_{g}(4 m)}$ in $\mathbb{H}_{g}^{*}$, where

$$
\mathbb{H}_{g}^{\tau \Gamma_{g}(4 m)}=\left\{\Omega \in \mathbb{H}_{g} \mid \tau(\Omega)=-\bar{\Omega}=\gamma \cdot \Omega \quad \text { for some } \gamma \in \Gamma_{g}(4 m)\right\}
$$

denotes the set of $\Gamma_{g}(4 m)$-real points of $\mathbb{H}_{g}$.

Proof. The proof can be found in [8, p. 23]. 
Theorem 5.4. Let $k$ be a positive integer with $k \geq 2$. Let $(\mathscr{F}, \gamma)$ be a $\Gamma_{g}\left(2^{k}\right)$ real boundary pair. Then there exists $\gamma_{1} \in \Gamma_{g}\left(2^{k}\right)$ such that $\mathscr{F}^{\tau \gamma}=\mathscr{F}^{\tau \gamma_{1}}$ and $\mathscr{F}^{\tau \gamma}$ is contained in the closure $\overline{\mathbb{H}_{g}^{\tau \gamma_{1}}}$ of $\mathbb{H}_{g}^{\tau \gamma_{1}}$ in $\mathbb{H}_{g}^{*}$.

Proof. The proof can be found in [8, pp. 23-26].

We may summarize the above results as follows. The Baily-Borel compactification $V(N)=\Gamma_{g}(N) \backslash \mathbb{H}_{g}^{*}$ with $N=4 m$ is stratified by finitely many strata of the form $\pi_{B B}(\mathscr{F})$, where $\mathscr{F}$ is a rational boundary component. Each such strata is isomorphic to the standard rational boundary component $\mathscr{F}_{s} \cong \mathbb{H}_{s}$. The stratum $\pi_{B B}(\mathscr{F})$ is called a boundary stratum of degree $s$. Let $V(N)^{r}$ denote the union of all boundary strata of rank $g-r$. We define

$$
V(N)_{\mathbb{R}}^{r}:=V(N)^{r} \cap V(N)_{\mathbb{R}} .
$$

According to Theorem 5.4, we have

$$
V(N)_{\mathbb{R}}^{0} \cup V(N)_{\mathbb{R}}^{1} \subset \overline{X(N)_{\mathbb{R}}} \subset V(N)_{\mathbb{R}},
$$

where $\overline{X(N)_{\mathbb{R}}}$ denotes the closure of $X(N)_{\mathbb{R}}$ in $V(N)$.

\section{Polarized real tori}

In this section we introduce the notion of polarized real tori.

First we review the properties of real tori briefly. We fix a positive integer $g$ in this section. Let $T=\mathbb{R}^{g} / \Lambda$ be a real torus of dimension $g$, where $\Lambda$ is a lattice in $\mathbb{R}^{g}$. $T$ has a unique structure of a smooth (or real analytic) manifold such that the canonical projection $p: \mathbb{R}^{g} \longrightarrow T$ is smooth (or real analytic). We fix the standard basis $\left\{e_{1}, \ldots, e_{g}\right\}$ for $\mathbb{R}^{g}$. We see that $\Lambda=\Pi \mathbb{Z}^{g}$ for some $\Pi \in G L(g, \mathbb{R})$. A matrix $\Pi$ is called a period matrix for $T$. Let $\mathbb{C}_{1}^{*}=\{z \in \mathbb{C}|| z \mid=1\}$ be a circle. Since $T$ is homeomorphic to $\mathbb{C}_{1}^{*} \times \cdots \times \mathbb{C}_{1}^{*}(g$ times), the fundamental group is

$$
\pi_{1}(T) \cong \pi_{1}\left(\mathbb{C}_{1}^{*}\right) \times \cdots \times \pi_{1}\left(\mathbb{C}_{1}^{*}\right) \cong \mathbb{Z}^{g} .
$$

We see that

$$
H_{k}(T, \mathbb{Z}) \cong \mathbb{Z}^{g} C_{k} \cong H^{k}(T, \mathbb{Z}), \quad k=0,1, \ldots, g
$$

and

$$
H^{*}(T, \mathbb{Z}) \cong \bigwedge H^{1}(T, \mathbb{Z}) \cong \bigwedge \mathbb{Z}^{g}
$$

Thus the Euler characteristic of $T$ is zero. The mapping class group $M C G(T)$ is

$$
M C G(T)=\operatorname{Aut}\left(\pi_{1}(T)\right)=\operatorname{Aut}\left(\mathbb{Z}^{g}\right)=G L(g, \mathbb{Z}) .
$$

It is known that any connected compact real manifold can be embedded into the Euclidean space $\mathbb{R}^{d}$ with large $d$. Thus a torus $T$ can be embedded in a real projective space $\mathbb{P}^{d}(\mathbb{R})$. Any connected compact abelian real Lie group is a real torus. Any two real tori of dimension $g$ are isomorphic as real Lie groups. We easily see that if $S$ is a connected closed subgroup of a real torus $T$, then $S$ and $T / S$ are real tori and $T \cong S \times T / S$. 
Let $T=V / \Lambda$ and $T^{\prime}=V^{\prime} / \Lambda^{\prime}$ be two real tori. A homomorphism $\phi: T \longrightarrow$ $T^{\prime}$ is a real analytic map compatible with the group structures. It is easily seen that a homomorphism $\phi: T \longrightarrow T^{\prime}$ can be lifted to a uniquely determined $\mathbb{R}$-linear map $\Phi: V \longrightarrow V^{\prime}$. This yields an injective homomorphism of abelian groups

$$
\tau_{a}: \operatorname{Hom}\left(T, T^{\prime}\right) \longrightarrow \operatorname{Hom}_{\mathbb{R}}\left(V, V^{\prime}\right), \quad \phi \longmapsto \Phi,
$$

where $\operatorname{Hom}\left(T, T^{\prime}\right)$ is the abelian group of all homomorphisms of $T$ into $T^{\prime}$ and $\operatorname{Hom}_{\mathbb{R}}\left(V, V^{\prime}\right)$ is the abelian group of all $\mathbb{R}$-linear maps of $V$ into $V^{\prime}$. The above $\tau_{a}$ is called a real analytic representation of $\operatorname{Hom}\left(T, T^{\prime}\right)$. The restriction $\Phi_{\Lambda}$ of $\Phi$ to $\Lambda$ is $\mathbb{Z}$-linear. $\Phi_{\Lambda}$ determines $\Phi$ and $\phi$ completely. Thus we get an injective homomorphism

$$
\tau_{r}: \operatorname{Hom}\left(T, T^{\prime}\right) \longrightarrow \operatorname{Hom}_{\mathbb{Z}}\left(\Lambda, \Lambda^{\prime}\right), \quad \phi \longmapsto \Phi_{\Lambda},
$$

called the rational representation of $\operatorname{Hom}\left(T, T^{\prime}\right)$.

Lemma 6.1. Let $\phi: T \longrightarrow T^{\prime}$ be a homomorphism of real tori. Then

(1) the image $\operatorname{Im} \phi$ is a real subtorus of $T^{\prime}$;

(2) the kernel ker $\phi$ of $\phi$ is a closed subgroup of $T$ and the identity component $(\operatorname{ker} \phi)_{0}$ of $\operatorname{ker} \phi$ is a real subtorus of $T$ of finite index in $\operatorname{ker} \phi$.

Proof. It follows from the fact that a connected compact abelian real Lie group is a real torus. Since $\operatorname{ker} \phi$ is compact, $\operatorname{ker} \phi$ has only a finite number of connected components.

A surjective homomorphism $\phi: T \longrightarrow T^{\prime}$ of real tori with finite kernel is called a real isogeny or simply an isogeny. The exponent $e(\phi)$ of an isogeny $\phi$ is defined to be the exponent of the finite group $\operatorname{ker} \phi$, that is, the smallest positive integer $e$ such that $e \cdot x=0$ for all $x \in \operatorname{ker} \phi$. Two real tori are said to be isogenous if there is an isogeny between them. It is clear that a homomorphism $\phi: T \longrightarrow T^{\prime}$ is an isogeny if and only if it is surjective and $\operatorname{dim} T=\operatorname{dim} T^{\prime}$. We can see that if $\Gamma \subset T$ is a finite subgroup, the quotient space $T / \Gamma$ is a real torus and the natural projection $p_{\Gamma}: T \longrightarrow T / \Gamma$ is an isogeny.

For a homomorphism $\phi: T \longrightarrow T^{\prime}$ of real tori, we define the degree of $\phi$ to be

$$
\operatorname{deg} \phi:=\left\{\begin{array}{cl}
\operatorname{ord}(\operatorname{ker} \phi) & \text { if } \operatorname{ker} \phi \text { is finite } \\
0 & \text { otherwise. }
\end{array}\right.
$$

Let $T=V / \Lambda$ be a real torus of dimension $g$. For any nonzero integer $n \in \mathbb{Z}$, we define the isogeny $n_{T}: T \longrightarrow T$ by $n_{T}(x):=n \cdot x$ for all $x \in T$. The kernel $T(n)$ of $n_{T}$ is called the group of $n$-division points of $T$. It is easily seen that $T(n) \cong(\mathbb{Z} / n \mathbb{Z})^{g}$ because $\operatorname{ker} n_{T}=\frac{1}{n} \Lambda / \Lambda \cong \Lambda / n \Lambda \cong(\mathbb{Z} / n \mathbb{Z})^{g}$. So $\operatorname{deg} n_{T}=n^{g}$.

We put

and

$$
\operatorname{Hom}_{\mathbb{Q}}\left(T, T^{\prime}\right):=\operatorname{Hom}\left(T, T^{\prime}\right) \otimes_{\mathbb{Z}} \mathbb{Q}
$$

$$
\operatorname{End}(T):=\operatorname{Hom}(T, T), \quad \operatorname{End}_{\mathbb{Q}}(T):=\operatorname{End}(T) \otimes_{\mathbb{Z}} \mathbb{Q}
$$


For any $\alpha \in \mathbb{Q}$ and $\phi \in \operatorname{Hom}\left(T, T^{\prime}\right)$, we define the degree of $\alpha \phi \in \operatorname{Hom}_{\mathbb{Q}}\left(T, T^{\prime}\right)$ by

$$
\operatorname{deg}(\alpha \phi):=\alpha^{g} \operatorname{deg} \phi .
$$

Lemma 6.2. For any isogeny $\phi: T \longrightarrow T^{\prime}$ of real tori with exponent $e$, there exists an isogeny $\psi: T^{\prime} \longrightarrow T$, unique up to isomorphisms, such that $\psi \circ \phi=e_{T}$ and $\phi \circ \psi=e_{T^{\prime}}$.

Proof. Since $\operatorname{ker} \phi \subseteq \operatorname{ker} e_{T}$, there exists a unique map $\psi: T^{\prime} \longrightarrow T$ such that $\psi \circ \phi=e_{T}$. It is easy to see that $\psi$ is also an isogeny and that $\operatorname{ker} \psi \subseteq \operatorname{ker} e_{T^{\prime}}$. Therefore there is a unique isogeny $\phi^{\prime}: T^{\prime} \longrightarrow T$ such that $\phi^{\prime} \circ \psi=e_{T^{\prime}}$. Since

$$
\phi^{\prime} \circ e_{T}=\phi^{\prime} \circ \psi \circ \phi=e_{T^{\prime}} \circ \phi=\phi \circ e_{T}
$$

and $e_{T}$ is surjective, we have $\phi^{\prime}=\phi$. Hence we obtain $\psi \circ \phi=e_{T}$ and $\phi \circ \psi=$ $e_{T^{\prime}}$.

According to Lemma 6.2, we see that isogenies define an equivalence relation on the set of real tori, and that an element in $\operatorname{End}(T)$ is an isogeny if and only if it is invertible in $\operatorname{End}_{\mathbb{Q}}(T)$.

For a real torus $T=V / \Lambda$ of dimension $g$, we put $V^{*}:=\operatorname{Hom}_{\mathbb{R}}(V, \mathbb{R})$. Then the following canonical $\mathbb{R}$-bilinear form

$$
\langle,\rangle_{T}: V^{*} \times V \longrightarrow \mathbb{R}, \quad\langle\ell, v\rangle_{T}:=\ell(v), \ell \in V^{*}, v \in V
$$

is non-degenerate. Thus the set

$$
\widehat{\Lambda}:=\left\{\ell \in V^{*} \mid\langle\ell, \Lambda\rangle_{T} \subseteq \mathbb{Z}\right\}
$$

is a lattice in $V^{*}$. The quotient

$$
\widehat{T}:=V^{*} / \widehat{\Lambda}
$$

is a real torus of dimension $g$ which is called the dual real torus of $T$. Identifying $V$ with the space of $\mathbb{R}$-linear forms $V^{*} \longrightarrow \mathbb{R}$ by double duality, the nondegeneracy of $\langle,\rangle_{T}$ implies that $\Lambda$ is the lattice in $V$ dual to $\widehat{\Lambda}$. Therefore we get

$$
\widehat{\widehat{T}}=T \text {. }
$$

Let $\phi: T_{1} \longrightarrow T_{2}$ be a homomorphism of real tori with $T_{i}=V_{i} / \Lambda_{i}(i=1,2)$ and with real analytic representation $\Phi: V_{1} \longrightarrow V_{2}$. Since the dual map $\Phi^{*}$ : $V_{2}^{*} \longrightarrow V_{1}^{*}$ satisfies the condition $\Phi^{*}\left(\widehat{\Lambda}_{2}\right) \subseteq \widehat{\Lambda}_{1}, \Phi^{*}$ induces a homomorphism, called the dual map

$$
\widehat{\phi}: \widehat{T}_{2} \longrightarrow \widehat{T}_{1} .
$$

If $\psi: T_{2} \longrightarrow T_{3}$ is another homomorphism of real tori, then we get

$$
\widehat{\psi \circ \phi}=\widehat{\phi} \circ \widehat{\psi} \text {. }
$$

If $\phi: T_{1} \longrightarrow T_{2}$ is an isogeny of real tori, then dual map $\widehat{\phi}: \widehat{T}_{2} \longrightarrow \widehat{T}_{1}$ is also an isogeny. 
Definition. A real torus $T=\mathbb{R}^{g} / \Lambda$ with a lattice $\Lambda$ in $\mathbb{R}^{g}$ is said to be polarized if the associated complex torus $\mathfrak{A}=\mathbb{C}^{g} / L$ is a polarized real abelian variety, where $L=\mathbb{Z}^{g}+i \Lambda$ is a lattice in $\mathbb{C}^{g}$. Moreover if $\mathfrak{A}$ is a principally polarized real abelian variety, $T$ is said to be principally polarized. Let $\Phi: T \longrightarrow \mathfrak{A}$ be the smooth embedding of $T$ into $\mathfrak{A}$ defined by

$$
\Phi(v+\Lambda):=i v+L, \quad v \in \mathbb{R}^{g} .
$$

Let $\mathfrak{L}$ be a polarization of $\mathfrak{A}$, that is, an ample line bundle over $\mathfrak{A}$. The pullback $\Phi^{*} \mathfrak{L}$ is called a polarization of $T$. We say that a pair $\left(T, \Phi^{*} \mathfrak{L}\right)$ is a polarized real torus.

Example 6.1. Let $Y \in \mathcal{P}_{g}$ be a $g \times g$ positive definite symmetric real matrix. Then $\Lambda_{Y}=Y \mathbb{Z}^{g}$ is a lattice in $\mathbb{R}^{g}$. Then the $g$-dimensional torus $T_{Y}=\mathbb{R}^{g} / \Lambda_{Y}$ is a principally polarized real torus. Indeed,

$$
\mathfrak{A}_{Y}=\mathbb{C}^{g} / L_{Y}, \quad L_{Y}=\mathbb{Z}^{g}+i \Lambda_{Y}
$$

is a princially polarized real abelian variety. Its corresponding hermitian form $H_{Y}$ is given by

$$
H_{Y}(x, y)=E_{Y}(i x, y)+i E_{Y}(x, y)={ }^{t} x Y^{-1} \bar{y}, \quad x, y \in \mathbb{C}^{g},
$$

where $E_{Y}$ denotes the imaginary part of $H_{Y}$. It is easily checked that $H_{Y}$ is positive definite and $E_{Y}\left(L_{Y} \times L_{Y}\right) \subset \mathbb{Z}$ (cf. [17, pp. 29-30]). The real structure $\sigma_{Y}$ on $\mathfrak{A}_{Y}$ is a complex conjugation.

Example 6.2. Let $Q=\left(\begin{array}{cc}\sqrt{2} & \sqrt{3} \\ \sqrt{3} & -\sqrt{5}\end{array}\right)$ be a $2 \times 2$ symmetric real matrix of signature $(1,1)$. Then $\Lambda_{Q}=Q \mathbb{Z}^{2}$ is a lattice in $\mathbb{R}^{2}$. Then the real torus $T_{Q}=\mathbb{R}^{2} / \Lambda_{Q}$ is not polarized because the associated complex torus $\mathfrak{A}_{Q}=\mathbb{C}^{2} / L_{Q}$ is not an abelian variety, where $L_{Q}=\mathbb{Z}^{2}+i \Lambda_{Q}$ is a lattice in $\mathbb{C}^{2}$.

Definition. Two polarized tori $T_{1}=\mathbb{R}^{g} / \Lambda_{1}$ and $T_{2}=\mathbb{R}^{g} / \Lambda_{2}$ are said to be isomorphic if the associated polarized real abelian varieties $\mathfrak{A}_{1}=\mathbb{C}^{g} / L_{1}$ and $\mathfrak{A}_{2}=\mathbb{C}^{g} / L_{2}$ are isomorphic, where $L_{i}=\mathbb{Z}^{g}+i \Lambda_{i}(i=1,2)$, more precisely, if there exists a linear isomorphism $\varphi: \mathbb{C}^{g} \longrightarrow \mathbb{C}^{g}$ such that

$$
\begin{aligned}
\varphi\left(L_{1}\right) & =L_{2}, \\
\varphi_{*}\left(E_{1}\right) & =E_{2}, \\
\varphi_{*}\left(\sigma_{1}\right) & =\varphi \circ \sigma_{1} \circ \varphi^{-1}=\sigma_{2},
\end{aligned}
$$

where $E_{1}$ and $E_{2}$ are polarizations of $\mathfrak{A}_{1}$ and $\mathfrak{A}_{2}$ respectively, and $\sigma_{1}$ and $\sigma_{2}$ denotes the real structures (in fact complex conjugations) on $\mathfrak{A}_{1}$ and $\mathfrak{A}_{2}$ respectively.

Example 6.3. Let $Y_{1}$ and $Y_{2}$ be two $g \times g$ positive definite symmetric real matrices. Then $\Lambda_{i}:=Y_{i} \mathbb{Z}^{g}$ is a lattice in $\mathbb{R}^{g}(i=1,2)$. We let

$$
T_{i}:=\mathbb{R}^{g} / \Lambda_{i}, \quad i=1,2
$$


be real tori of dimension $g$. Then according to Example 6.1, $T_{1}$ and $T_{2}$ are principally polarized real tori. We see that $T_{1}$ is isomorphic to $T_{2}$ as polarized real tori if and only if there is an element $A \in G L(g, \mathbb{Z})$ such that $Y_{2}=A Y_{1}{ }^{t} A$.

Example 6.4. Let $Y=\left(\begin{array}{cc}\sqrt{2} & \sqrt{3} \\ \sqrt{3} & \sqrt{5}\end{array}\right)$. Let $T_{Y}=\mathbb{R}^{2} / \Lambda_{Y}$ be a two dimensional principally polarized torus, where $\Lambda_{Y}=Y \mathbb{Z}^{2}$ is a lattice in $\mathbb{R}^{2}$. Let $T_{Q}$ be the torus in Example 6.2. Then $T_{Y}$ is diffeomorphic to $T_{Q}$. But $T_{Q}$ is not polarized. $T_{Y}$ admits a differentiable embedding into a complex projective space but $T_{Q}$ does not.

Let $Y \in \mathcal{P}_{g}$ be a $g \times g$ positive definite symmetric real matrix. Then $\Lambda_{Y}=$ $Y \mathbb{Z}^{g}$ is a lattice in $\mathbb{R}^{g}$. We already showed that the $g$-dimensional torus $T_{Y}=$ $\mathbb{R}^{g} / \Lambda_{Y}$ is a principally polarized real torus (cf. Example 6.1). We know that the following complex torus

$$
\mathfrak{A}_{Y}=\mathbb{C}^{g} / L_{Y}, \quad L_{Y}=\mathbb{Z}^{g}+i \Lambda_{Y}
$$

is a princially polarized real abelian variety. We define a map $\Phi_{Y}: T_{Y} \longrightarrow \mathfrak{A}_{Y}$ by

$$
\Phi_{Y}\left(a+\Lambda_{Y}\right):=i a+L_{Y}, \quad a \in \mathbb{R}^{g} .
$$

Then $\Phi_{Y}$ is well defined and is an injective smooth map. Therefore $T_{Y}$ is smoothly embedded into a complex projective space and hence into a real projective space because $\mathfrak{A}_{Y}$ can be holomorphically embedded into a complex projective space (cf. [17, pp. 29-30]).

Let $\mathfrak{A}=\mathbb{C}^{g} / L$ and $\mathfrak{A}^{\prime}=\mathbb{C}^{g^{\prime}} / L^{\prime}$ be two abelian complex tori of dimension $g$ and dimension $g^{\prime}$ respectively, where $L$ (resp. $L^{\prime}$ ) is a lattice in $\mathbb{C}^{g}$ (resp. $\mathbb{C}^{g^{\prime}}$ ). A homomorphism $f: \mathfrak{A} \longrightarrow \mathfrak{A}^{\prime}$ lifts to a uniquely determined $\mathbb{C}$-linear map $F: \mathbb{C}^{g} \longrightarrow \mathbb{C}^{g^{\prime}}$. This yields an injective homomorphism

$$
\rho_{a}: \operatorname{Hom}\left(\mathfrak{A}, \mathfrak{A}^{\prime}\right) \longrightarrow \operatorname{Hom}_{\mathbb{C}}\left(\mathbb{C}^{g}, \mathbb{C}^{g^{\prime}}\right)=\mathbb{C}^{\left(g^{\prime}, g\right)}, \quad f \longmapsto F=\rho_{a}(f) .
$$

Its restriction $\left.F\right|_{L}$ to the lattice $L$ is $\mathbb{Z}$-linear and determines $F$ and $f$ completely. Therefore we get an injective homomorphism

$$
\rho_{r}: \operatorname{Hom}\left(\mathfrak{A}, \mathfrak{A}^{\prime}\right) \longrightarrow \operatorname{Hom}_{\mathbb{Z}}\left(L, L^{\prime}\right),\left.\quad f \longmapsto F\right|_{L}
$$

Let $\widetilde{\Pi} \in \mathbb{C}^{(g, 2 g)}$ and $\widetilde{\Pi}^{\prime} \in \mathbb{C}^{\left(g^{\prime}, 2 g^{\prime}\right)}$ be period matrices for $\mathfrak{A}$ and $\mathfrak{A}^{\prime}$ respectively. With respect to the chosen bases, $\rho_{a}(f)$ (resp. $\rho_{r}(f)$ ) can be considered as a matrix in $\mathbb{C}^{\left(g^{\prime}, g\right)}$ (resp. $\left.\mathbb{Z}^{\left(2 g^{\prime}, 2 g\right)}\right)$. We have the following diagram:

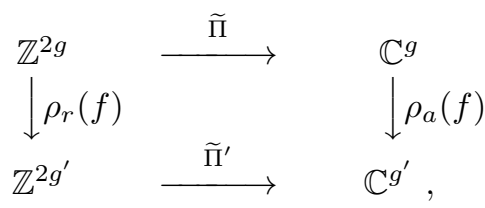

that is, by the equation

$$
\rho_{a}(f) \widetilde{\Pi}=\widetilde{\Pi}^{\prime} \rho_{r}(f)
$$


Conversely any two matrices $A \in \mathbb{C}^{\left(g^{\prime}, g\right)}$ and $R \in \mathbb{Z}^{\left(2 g^{\prime}, 2 g\right)}$ satisfying the equation $A \widetilde{\Pi}=\widetilde{\Pi}^{\prime} R$ define a homomorphism $\mathfrak{A} \longrightarrow \mathfrak{A}^{\prime}$.

For two real tori $T_{1}$ and $T_{2}$ of dimension $g_{1}$ and dimension $g_{2}$ respectively, we let $\operatorname{Ext}\left(T_{2}, T_{1}\right)$ be the set of all isomorphism classes of extensions of $T_{2}$ by $T_{1}$ up to real analytic isomorphism. Since any two real tori of dimension $g_{1}+g_{2}$ are isomorphic as real analytic real Lie groups, $\operatorname{Ext}\left(T_{2}, T_{1}\right)$ is trivial. This leads us to consider polarized real tori $T_{1}$ and $T_{2}$ with $T_{i}=\mathbb{R}^{g_{i}} / \Lambda_{i}(i=1,2)$. Here $\Lambda_{i}$ is a lattice in $\mathbb{R}^{g_{i}}$ for $i=1,2$. Let $\mathfrak{A}_{1}$ and $\mathfrak{A}_{2}$ be the polarized real abelian varieties associated to $T_{1}$ and $T_{2}$ respectively, that is,

$$
\mathfrak{A}_{i}=\mathbb{C}^{g_{i}} / L_{i}, \quad L_{i}=\mathbb{Z}^{g_{i}}+\Lambda_{i} \mathbb{Z}^{g_{i}}, \quad i=1,2 .
$$

Let $\operatorname{Ext}\left(T_{2}, T_{1}\right)_{\mathrm{pt}}$ be the set of all isomorphism classes of extensions of $\mathfrak{A}_{2}$ by $\mathfrak{A}_{1}$. We can show that a homomorphism $\phi: \mathfrak{A}_{2}^{\prime} \longrightarrow \mathfrak{A}_{2}$ such that $\mathfrak{A}_{2}^{\prime}$ is the real abelian variety associated to a polarized real torus $T_{2}^{\prime}$ induces a map

$$
\phi^{*}: \operatorname{Ext}\left(T_{2}, T_{1}\right)_{\mathrm{pt}} \longrightarrow \operatorname{Ext}\left(T_{2}^{\prime}, T_{1}\right)_{\mathrm{pt}}
$$

and that a homomorphism $\psi: \mathfrak{A}_{1} \longrightarrow \mathfrak{A}_{1}^{\prime}$ such that $\mathfrak{A}_{1}^{\prime}$ is the real abelian variety associated to a polarized real torus $T_{1}^{\prime}$ induces a map

$$
\psi_{*}: \operatorname{Ext}\left(T_{2}, T_{1}\right)_{\mathrm{pt}} \longrightarrow \operatorname{Ext}\left(T_{2}, T_{1}^{\prime}\right)_{\mathrm{pt}} .
$$

Indeed, if

$$
e: \quad 0 \longrightarrow \mathfrak{A}_{1} \stackrel{\iota}{\longrightarrow} \mathfrak{A} \stackrel{p}{\longrightarrow} \mathfrak{A}_{2} \longrightarrow 0
$$

is an extension in $\operatorname{Ext}\left(T_{2}, T_{1}\right)_{\mathrm{pt}}$, the image $\phi^{*}(e)$ is defined to be the identity component of the kernel of the homomorphism $C_{p, \phi}: \mathfrak{A} \times \mathfrak{A}_{2}^{\prime} \longrightarrow \mathfrak{A}_{2}$ defined by

$$
C_{p, \phi}(x, y):=p(x)-\phi(y), \quad x \in \mathfrak{A}, y \in \mathfrak{A}_{2}^{\prime} .
$$

The dualization of the exact sequence (6.7) gives an element $\hat{e} \in \operatorname{Ext}\left(\widehat{\mathfrak{A}}_{1}, \widehat{\mathfrak{A}}_{2}\right)$. We define

$$
\psi_{*}(e):=\widehat{\widehat{\psi}^{*}(\hat{e})} \quad \in \operatorname{Ext}\left(T_{2}, T_{1}^{\prime}\right)_{\mathrm{pt}}=\operatorname{Ext}\left(\mathfrak{A}_{2}, \mathfrak{A}_{1}^{\prime}\right) .
$$

Therefore $\operatorname{Ext}(,)_{\mathrm{pt}}$ is a functor which is contravariant in the first and covariant in the second argument.

We can equip the set $\operatorname{Ext}\left(T_{2}, T_{1}\right)_{\mathrm{pt}}$ with the canonical group structure as follows: Let $e$ and $e_{\diamond}$ be the extensions in $\operatorname{Ext}\left(T_{2}, T_{1}\right)_{\mathrm{pt}}$ which are represented by the exact sequence (6.7) and the following exact sequence

$$
e_{\diamond}: \quad 0 \longrightarrow \mathfrak{A}_{1} \longrightarrow \mathfrak{A}_{\diamond} \longrightarrow \mathfrak{A}_{2} \longrightarrow 0 .
$$

The product $e \times e_{\diamond}$ is represented by the exact sequence

$$
e \times e_{\diamond}: \quad 0 \longrightarrow \mathfrak{A}_{1} \times \mathfrak{A}_{1} \longrightarrow \mathfrak{A} \times \mathfrak{A}_{\diamond} \longrightarrow \mathfrak{A}_{2} \times \mathfrak{A}_{2} \longrightarrow 0 .
$$

If $\Delta: \mathfrak{A}_{2} \longrightarrow \mathfrak{A}_{2} \times \mathfrak{A}_{2}$ is the diagonal map, $x \longmapsto(x, x), x \in \mathfrak{A}_{2}$ and $\mu$ : $T_{1} \times T_{1} \longrightarrow T_{1}$ is the addition map, $(s, t) \longmapsto s+t, s, t \in \mathfrak{A}_{1}$, the sum $e+e_{\diamond}$ 
is defined to be the image of $e \times e_{\diamond}$ under the composition

$$
\operatorname{Ext}\left(T_{2} \times T_{2}, T_{1} \times T_{1}\right)_{\mathrm{pt}} \stackrel{\Delta^{*}}{\longrightarrow} \operatorname{Ext}\left(T_{2}, T_{1} \times T_{1}\right)_{\mathrm{pt}} \stackrel{\mu_{*}}{\longrightarrow} \operatorname{Ext}\left(T_{2}, T_{1}\right)_{\mathrm{pt}},
$$

that is,

$$
e+e_{\diamond}:=\mu_{*} \Delta^{*}\left(e \times e_{\diamond}\right) .
$$

We can show that $\operatorname{Ext}\left(T_{2}, T_{1}\right)_{\mathrm{pt}}$ is an abelian group with respect to the addition (6.9) (cf. [5]).

Now we describe the group $\operatorname{Ext}\left(T_{2}, T_{1}\right)_{\mathrm{pt}}$ in terms of period matrices. First we fix period matrices $\Pi_{1}$ and $\Pi_{2}$ for $T_{1}$ and $T_{2}$ respectively, that is, $\Lambda_{i}=\Pi_{i} \mathbb{Z}^{g_{i}}$ for $i=1,2$. We know that $\Pi_{i} \in G L\left(g_{i}, \mathbb{R}\right)$ for $i=1,2$. To each extension

$$
e: \quad 0 \longrightarrow \mathfrak{A}_{1} \longrightarrow \mathfrak{A} \longrightarrow \mathfrak{A}_{2} \longrightarrow 0
$$

in $\operatorname{Ext}\left(T_{2}, T_{1}\right)_{\mathrm{pt}}$, there is associated a period matrix for $\mathfrak{A}$ of the form

$$
\left(\begin{array}{cc}
\widetilde{\Pi}_{1} & \sigma \\
0 & \widetilde{\Pi}_{2}
\end{array}\right), \quad \widetilde{\Pi}_{i}=\left(I_{g_{i}}, \Pi_{i}\right) \text { for } i=1,2, \quad \sigma \in \mathbb{C}^{\left(g_{1}, 2 g_{2}\right)} .
$$

Conversely it is obvious that for any $\sigma \in \mathbb{C}^{\left(g_{1}, 2 g_{2}\right)}$, the matrix of the form (6.10) is a period matrix defining an extension of $\mathfrak{A}_{2}$ by $\mathfrak{A}_{1}$ in $\operatorname{Ext}\left(T_{2}, T_{1}\right)_{\mathrm{pt}}$.

Lemma 6.3. Let $\sigma$ and $\sigma^{\prime}$ be elements in $\mathbb{C}^{\left(g_{1}, 2 g_{2}\right)}$. Suppose that $\Pi_{1}$ and $\Pi_{2}$ are period matrices for polarized real tori $T_{1}$ and $T_{2}$ respectively. Then the period matrices

$$
\widetilde{\Pi}_{\sigma}=\left(\begin{array}{cc}
\widetilde{\Pi}_{1} & \sigma \\
0 & \widetilde{\Pi}_{2}
\end{array}\right) \quad \text { and } \quad \widetilde{\Pi}_{\sigma^{\prime}}=\left(\begin{array}{cc}
\widetilde{\Pi}_{1} & \sigma^{\prime} \\
0 & \widetilde{\Pi}_{2}
\end{array}\right), \quad \widetilde{\Pi}_{i}=\left(I_{g_{i}}, \Pi_{i}\right) \text { for } i=1,2
$$

define isomorphic extensions of $\mathfrak{A}_{2}$ by $\mathfrak{A}_{1}$ in $\operatorname{Ext}\left(T_{2}, T_{1}\right)_{\mathrm{pt}}$ if and only if

$$
\sigma^{\prime}=\sigma+\widetilde{\Pi}_{1} M+A \widetilde{\Pi}_{2}
$$

with some $M \in \mathbb{Z}^{\left(2 g_{1}, 2 g_{2}\right)}$ and $A \in \mathbb{C}^{\left(g_{1}, g_{2}\right)}$.

Proof. Let $\widetilde{\Pi}_{\sigma}$ and $\widetilde{\Pi}_{\sigma^{\prime}}$ define isomorphic extensions $e$ and $e^{\prime}$ of $\mathfrak{A}_{2}$ by $\mathfrak{A}_{1}$ :

$$
\begin{aligned}
& e: 0 \longrightarrow \mathfrak{A}_{1} \longrightarrow \mathfrak{A} \longrightarrow \mathfrak{A}_{2} \longrightarrow 0 \\
& e^{\prime}: 0 \longrightarrow \|_{\mathfrak{A}_{1}} \longrightarrow \begin{array}{l}
\downarrow f \\
\mathfrak{A}^{\prime}
\end{array} \longrightarrow \mathfrak{A}_{2} \longrightarrow 0
\end{aligned}
$$

Then we have the following commutative diagram:

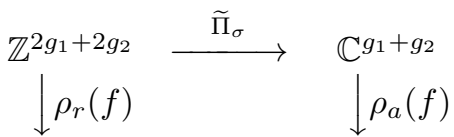

$$
\begin{aligned}
& \mathbb{Z}^{2 g_{1}+2 g_{2}} \stackrel{\widetilde{\Pi}_{\sigma^{\prime}}}{\longrightarrow} \mathbb{C}^{g_{1}+g_{2}}
\end{aligned}
$$


Therefore there are $A \in \mathbb{C}^{\left(g_{1}, g_{2}\right)}$ and $M \in \mathbb{Z}^{\left(2 g_{1}, 2 g_{2}\right)}$ that satisfy the following equation

$$
\left(\begin{array}{cc}
I_{g_{1}} & A \\
0 & I_{g_{2}}
\end{array}\right) \widetilde{\Pi}_{\sigma}=\widetilde{\Pi}_{\sigma^{\prime}}\left(\begin{array}{cc}
I_{g_{1}} & -M \\
0 & I_{g_{2}}
\end{array}\right) .
$$

We obtain the equation (6.11) from the equation (6.12).

Conversely if we have $\sigma$ and $\sigma^{\prime}$ in $\mathbb{C}^{\left(g_{1}, g_{2}\right)}$ satisfying the equation (6.11), then we see easily that $\widetilde{\Pi}_{\sigma}$ and $\widetilde{\Pi}_{\sigma^{\prime}}$ define isomorphic extensions of $\mathfrak{A}_{2}$ by $\mathfrak{A}_{1}$.

Proposition 6.1. Let $\sigma$ and $\sigma^{\prime}$ be elements in $\mathbb{C}^{\left(g_{1}, 2 g_{2}\right)}$. Suppose that $\Pi_{1}$ and $\Pi_{2}$ are period matrices for real tori $T_{1}$ and $T_{2}$ respectively. Assume that the following period matrices

$$
\widetilde{\Pi}_{\sigma}=\left(\begin{array}{cc}
\widetilde{\Pi}_{1} & \sigma \\
0 & \widetilde{\Pi}_{2}
\end{array}\right) \quad \text { and } \quad \widetilde{\Pi}_{\sigma^{\prime}}=\left(\begin{array}{cc}
\widetilde{\Pi}_{1} & \sigma^{\prime} \\
0 & \widetilde{\Pi}_{2}
\end{array}\right), \quad \widetilde{\Pi}_{i}=\left(I_{g_{i}}, \Pi_{i}\right) \text { for } i=1,2
$$

define extensions e and $e^{\prime}$ of $\mathfrak{A}_{2}$ by $\mathfrak{A}_{1}$ in $\operatorname{Ext}\left(T_{2}, T_{1}\right)_{\mathrm{pt}}$. Then the period matrix

$$
\widetilde{\Pi}_{\sigma+\sigma^{\prime}}=\left(\begin{array}{cc}
\widetilde{\Pi}_{1} & \sigma+\sigma^{\prime} \\
0 & \widetilde{\Pi}_{2}
\end{array}\right)
$$

defines the extension $e+e^{\prime}$ in $\operatorname{Ext}\left(T_{2}, T_{1}\right)_{\mathrm{pt}}$.

Proof. We denote

$$
\mathfrak{A}=\mathbb{C}^{g_{1}+g_{2}} / \widetilde{\Pi}_{\sigma} \mathbb{Z}^{2 g_{1}+2 g_{2}} \text { and } \mathfrak{A}^{\prime}=\mathbb{C}^{g_{1}+g_{2}} / \widetilde{\Pi}_{\sigma^{\prime}} \mathbb{Z}^{2 g_{1}+2 g_{2}} .
$$

Then we have the extensions

$$
e: \quad 0 \longrightarrow \mathfrak{A}_{1} \longrightarrow \mathfrak{A} \longrightarrow \mathfrak{A}_{2} \longrightarrow 0
$$

and

$$
e^{\prime}: \quad 0 \longrightarrow \mathfrak{A}_{1} \longrightarrow \mathfrak{A}^{\prime} \longrightarrow \mathfrak{A}_{2} \longrightarrow 0
$$

in $\operatorname{Ext}\left(T_{2}, T_{1}\right)_{\mathrm{pt}}$. The complex torus $\mathfrak{A} \times \mathfrak{A}^{\prime}$ defined by the extension $e \times e^{\prime}$ in $\operatorname{Ext}\left(\mathfrak{A}_{2} \times \mathfrak{A}_{2}, \mathfrak{A}_{1} \times \mathfrak{A}_{1}\right)$ is given by the period matrix

$$
\square_{1}=\left(\begin{array}{cccc}
\widetilde{\Pi}_{1} & 0 & \sigma & 0 \\
0 & \widetilde{\Pi}_{1} & 0 & \sigma^{\prime} \\
0 & 0 & \widetilde{\Pi}_{2} & 0 \\
0 & 0 & 0 & \widetilde{\Pi}_{2}
\end{array}\right) .
$$

Let $\Delta: \mathfrak{A}_{2} \longrightarrow \mathfrak{A}_{2} \times \mathfrak{A}_{2}$ be the diagonal map. Then we have the induced map $\Delta^{*}: \operatorname{Ext}\left(\mathfrak{A}_{2} \times \mathfrak{A}_{2}, \mathfrak{A}_{1} \times \mathfrak{A}_{1}\right) \longrightarrow \operatorname{Ext}\left(\mathfrak{A}_{2}, \mathfrak{A}_{1} \times \mathfrak{A}_{1}\right)$. If

$$
\Delta^{*}\left(e \times e^{\prime}\right): 0 \longrightarrow \mathfrak{A}_{1} \times \mathfrak{A}_{1} \longrightarrow S \longrightarrow \mathfrak{A}_{2} \longrightarrow 0
$$

is given, the complex torus $S$ is given by a period matrix of the form

$$
\square_{2}=\left(\begin{array}{ccc}
\widetilde{\Pi}_{1} & 0 & \alpha \\
0 & \widetilde{\Pi}_{1} & \beta \\
0 & 0 & \widetilde{\Pi}_{2}
\end{array}\right)
$$


with $\alpha \in \mathbb{C}^{\left(g_{1}, 2 g_{2}\right)}$ and $\beta \in \mathbb{C}^{\left(g_{1}, 2 g_{2}\right)}$. The homomorphism

$\operatorname{Ext}\left(\mathfrak{A}_{2} \times \mathfrak{A}_{2}, \mathfrak{A}_{1} \times \mathfrak{A}_{1}\right) \longrightarrow \operatorname{Ext}\left(\mathfrak{A}_{2}, \mathfrak{A}_{1} \times \mathfrak{A}_{1}\right), \quad e \times e^{\prime} \longmapsto \Delta^{*}\left(e \times e^{\prime}\right)$

corresponds to a homomorphism $S \longmapsto \mathfrak{A} \times \mathfrak{A}^{\prime}$ of real tori given by the equation

$$
\square_{1} \cdot\left(\begin{array}{ccc}
I_{2 g_{1}} & 0 & M_{1} \\
0 & I_{2 g_{1}} & M_{2} \\
0 & 0 & I_{2 g_{2}} \\
0 & 0 & I_{2 g_{2}}
\end{array}\right)=\left(\begin{array}{ccc}
I_{g_{1}} & 0 & A_{1} \\
0 & I_{g_{1}} & A_{2} \\
0 & 0 & I_{g_{2}} \\
0 & 0 & I_{g_{2}}
\end{array}\right) \cdot \square_{2}
$$

Thus we have the equations

$$
\alpha=\sigma+\widetilde{\Pi}_{1} M_{1}-A_{1} \widetilde{\Pi}_{2} \quad \text { and } \quad \beta=\sigma^{\prime}+\widetilde{\Pi}_{1} M_{2}-A_{2} \widetilde{\Pi}_{2} .
$$

According to Lemma 6.3,

$$
\left(\begin{array}{ccc}
\widetilde{\Pi}_{1} & 0 & \sigma \\
0 & \widetilde{\Pi}_{1} & \sigma^{\prime} \\
0 & 0 & \widetilde{\Pi}_{2}
\end{array}\right)
$$

is also a period matrix for $S$ respectively $\Delta^{*}\left(e \times e^{\prime}\right)$. We denote

$$
e+e^{\prime}: 0 \longrightarrow \mathfrak{A}_{1} \longrightarrow \mathfrak{B} \longrightarrow \mathfrak{A}_{2} \longrightarrow 0 .
$$

A period matrix for $\mathfrak{B}$ is of the form

$$
\Pi_{\mathfrak{B}}:=\left(\begin{array}{cc}
\widetilde{\Pi}_{1} & \tau \\
0 & \widetilde{\Pi}_{2}
\end{array}\right), \quad \tau \in \mathbb{R}^{\left(g_{1}, g_{2}\right)} .
$$

The homomorphism $\mu_{*}: \Delta^{*}\left(e \times e^{\prime}\right) \longmapsto e+e^{\prime}$ defines a homomorphism $S \longmapsto \mathfrak{B}$ which is given by the equation

$$
\left(\begin{array}{ccc}
I_{g_{1}} & I_{g_{1}} & A \\
0 & 0 & I_{g_{2}}
\end{array}\right)\left(\begin{array}{ccc}
\widetilde{\Pi}_{1} & 0 & \sigma \\
0 & \widetilde{\Pi}_{1} & \sigma^{\prime} \\
0 & 0 & \widetilde{\Pi}_{2}
\end{array}\right)=\left(\begin{array}{cc}
\widetilde{\Pi}_{1} & \tau \\
0 & \widetilde{\Pi}_{2}
\end{array}\right)\left(\begin{array}{ccc}
I_{2 g_{1}} & I_{2 g_{1}} & M \\
0 & 0 & I_{2 g_{2}}
\end{array}\right)
$$

with $A \in \mathbb{C}^{\left(g_{1}, g_{2}\right)}$ and $M \in \mathbb{Z}^{\left(2 g_{1}, 2 g_{2}\right)}$. Comparing both sides in the equation (6.13), we obtain

$$
\tau=\sigma+\sigma^{\prime}-\widetilde{\Pi}_{1} M+A \widetilde{\Pi}_{2} .
$$

According to Lemma 6.3, we see that

$$
\widetilde{\Pi}_{\sigma+\sigma^{\prime}}=\left(\begin{array}{cc}
\widetilde{\Pi}_{1} & \sigma+\sigma^{\prime} \\
0 & \widetilde{\Pi}_{2}
\end{array}\right)
$$

is a period matrix for $\mathfrak{B}$, respectively $e+e^{\prime}$.

Let $T_{1}, T_{2}, \Pi_{1}, \Pi_{2}, \widetilde{\Pi}_{1}, \widetilde{\Pi}_{2}, \sigma, \sigma^{\prime}, \widetilde{\Pi}_{\sigma}$ and $\widetilde{\Pi}_{\sigma^{\prime}}$ be as above in Proposition 6.1 . We note that the assignment

$$
\sigma \longmapsto \mathbb{C}^{g_{1}+g_{2}} / \widetilde{\Pi}_{\sigma} \mathbb{Z}^{2 g_{1}+2 g_{2}}, \quad \sigma \in \mathbb{C}^{\left(g_{1}, 2 g_{2}\right)}
$$

induces a surjective homomorphism of abelian groups

$$
\Phi_{\Pi_{1}, \Pi_{2}}: \mathbb{C}^{\left(g_{1}, 2 g_{2}\right)} \longrightarrow \operatorname{Ext}\left(T_{2}, T_{1}\right)_{\mathrm{pt}} .
$$


According to Lemma 6.3 , we see that the kernel of $\Phi_{\Pi_{1}, \Pi_{2}}$ is given by

$$
\operatorname{ker} \Phi_{\Pi_{1}, \Pi_{2}}=\widetilde{\Pi}_{1} \mathbb{Z}^{\left(2 g_{1}, 2 g_{2}\right)}+\mathbb{C}^{\left(g_{1}, g_{2}\right)} \widetilde{\Pi}_{2} .
$$

Obviously the homomorphism $\Phi_{\Pi_{1}, \Pi_{2}}$ depends on the choice of the period matrices $\Pi_{1}$ and $\Pi_{2}$.

Proposition 6.2. Let $T_{1}$ and $T_{1}^{\prime}$ be polarized real tori of dimension $g_{1}$ and dimension $g_{1}^{\prime}$ with period matrices $\Pi_{1}$ and $\Pi_{1}^{\prime}$ respectively. Let $T_{2}$ and $T_{2}^{\prime}$ be polarized real tori of dimension $g_{2}$ and dimension $g_{2}^{\prime}$ with period matrices $\Pi_{2}$ and $\Pi_{2}^{\prime}$ respectively. Then

(a) for a homomorphism $f: \mathfrak{A}_{2}^{\prime} \longrightarrow \mathfrak{A}_{2}$ such that $\mathfrak{A}_{2}^{\prime}$ is the polarized real abelian variety associated to a polarized real torus $T_{2}^{\prime}$, the following diagram

commutes and

$$
\begin{array}{ccc}
\mathbb{C}^{\left(g_{1}, 2 g_{2}\right)} & \stackrel{\Phi_{\Pi_{1}, \Pi_{2}}}{\longrightarrow} & \operatorname{Ext}\left(T_{2}, T_{1}\right)_{\mathrm{pt}} \\
\downarrow \cdot \rho_{r}(f) & f^{*} \\
\mathbb{C}^{\left(g_{1}, 2 g_{2}^{\prime}\right)} & \stackrel{\Phi_{\Pi_{1}, \Pi_{2}^{\prime}}}{\longrightarrow} & \operatorname{Ext}\left(T_{2}^{\prime}, T_{1}\right)_{\mathrm{pt}}
\end{array}
$$

(b) for a homomorphism $h: \mathfrak{A}_{1} \longrightarrow \mathfrak{A}_{1}^{\prime}$ such that $\mathfrak{A}_{1}^{\prime}$ is the polarized real abelian variety associated to a polarized real torus $T_{1}^{\prime}$, the following diagram

$$
\begin{array}{ccc}
\mathbb{C}^{\left(g_{1}, 2 g_{2}\right)} & \stackrel{\Phi_{\Pi_{1}, \Pi_{2}}}{\longrightarrow} & \operatorname{Ext}\left(T_{2}, T_{1}\right)_{\mathrm{pt}} \\
\rho_{a}(h) \cdot \downarrow & h_{*} \\
\mathbb{C}^{\left(g_{1}^{\prime}, 2 g_{2}\right)} & \stackrel{\Phi_{\Pi_{1}^{\prime}, \Pi_{2}}}{\longrightarrow} & \operatorname{Ext}\left(T_{2}, T_{1}^{\prime}\right)_{\mathrm{pt}}
\end{array}
$$

commutes.

Proof. (a) For an extension $e \in \operatorname{Ext}\left(T_{2}, T_{1}\right)_{\mathrm{pt}}$ we choose $\sigma \in \mathbb{C}^{\left(g_{1}, 2 g_{2}\right)}$ with $\Phi_{\Pi_{1}, \Pi_{2}}(\sigma)=e$ and $\sigma^{\prime} \in \mathbb{C}^{\left(g_{1}, 2 g_{2}^{\prime}\right)}$ with $\Phi_{\Pi_{1}, \Pi_{2}^{\prime}}\left(\sigma^{\prime}\right)=f^{*}(e)$. We see that the following diagram with exact rows

$$
\begin{aligned}
& f^{*}(e): 0 \longrightarrow \mathfrak{A}_{1} \longrightarrow \mathfrak{A}^{\prime} \longrightarrow \mathfrak{A}_{2}^{\prime} \longrightarrow 0 \\
& e: 0 \quad 0 \quad \longrightarrow \quad \mathfrak{A}_{1} \longrightarrow \begin{array}{l}
\downarrow f^{*} \\
\mathfrak{A}
\end{array} \longrightarrow \begin{array}{l}
\downarrow f \\
\mathfrak{A}_{2} \longrightarrow 0
\end{array}
\end{aligned}
$$

commutes. Thus $\sigma$ and $\sigma^{\prime}$ are related by the equation

$$
\left(\begin{array}{cc}
I_{g_{1}} & A \\
0 & \rho_{a}(f)
\end{array}\right)\left(\begin{array}{cc}
\widetilde{\Pi}_{1} & \sigma^{\prime} \\
0 & \widetilde{\Pi}_{2}^{\prime}
\end{array}\right)=\left(\begin{array}{cc}
\widetilde{\Pi}_{1} & \sigma \\
0 & \widetilde{\Pi}_{2}
\end{array}\right)\left(\begin{array}{cc}
I_{2 g_{1}} & M \\
0 & \rho_{r}(f)
\end{array}\right)
$$

with $A \in \mathbb{C}^{\left(g_{1}, g_{2}^{\prime}\right)}$ and $M \in \mathbb{Z}^{\left(2 g_{1}, 2 g_{2}^{\prime}\right)}$. Comparing both sides in the equation (6.15), we get

$$
\sigma^{\prime}=\sigma \cdot \rho_{r}(f)+\widetilde{\Pi}_{1} M-A \widetilde{\Pi}_{2}^{\prime} .
$$

According to Lemma 6.3, we have

$$
\Phi_{\Pi_{1}, \Pi_{2}^{\prime}}\left(\sigma^{\prime}\right)=\Phi_{\Pi_{1}, \Pi_{2}^{\prime}}\left(\sigma \cdot \rho_{r}(f)\right)=f^{*}(e) .
$$


This completes the proof of (a).

(b) For an extension $e_{\diamond} \in \operatorname{Ext}\left(T_{2}, T_{1}\right)_{\mathrm{pt}}$ we choose $\sigma_{\diamond} \in \mathbb{C}^{\left(g_{1}, 2 g_{2}\right)}$ with $\Phi_{\Pi_{1}, \Pi_{2}}\left(\sigma_{\diamond}\right)=e_{\diamond}$ and $\sigma_{\diamond}^{\prime} \in \mathbb{C}^{\left(g_{1}^{\prime}, 2 g_{2}\right)}$ with $\Phi_{\Pi_{1}^{\prime}, \Pi_{2}}\left(\sigma_{\diamond}^{\prime}\right)=h_{*}\left(e_{\diamond}\right)$. We see that the following diagram with exact rows

$$
\begin{aligned}
& e_{\diamond}: 0 \longrightarrow \mathfrak{A}_{1} \longrightarrow \mathfrak{A}_{\diamond} \longrightarrow \mathfrak{A}_{2} \longrightarrow 0
\end{aligned}
$$

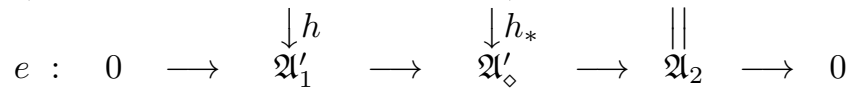

commutes. Thus $\sigma_{\diamond}$ and $\sigma_{\diamond}^{\prime}$ are related by the equation

$$
\left(\begin{array}{cc}
\widetilde{\Pi}_{1}^{\prime} & \sigma_{\diamond}^{\prime} \\
0 & \widetilde{\Pi}_{2}
\end{array}\right)\left(\begin{array}{cc}
\rho_{r}(h) & M_{\diamond} \\
0 & I_{2 g_{2}}
\end{array}\right)=\left(\begin{array}{cc}
\rho_{a}(h) & A_{\diamond} \\
0 & I_{g_{2}}
\end{array}\right)\left(\begin{array}{cc}
\widetilde{\Pi}_{1} & \sigma_{\diamond} \\
0 & \widetilde{\Pi}_{2}
\end{array}\right) \text {. }
$$

with $A_{\diamond} \in \mathbb{C}^{\left(g_{1}^{\prime}, g_{2}^{\prime}\right)}$ and $M_{\diamond} \in \mathbb{Z}^{\left(2 g_{1}^{\prime}, 2 g_{2}^{\prime}\right)}$. Comparing both sides in the equation (6.16), we get

$$
\sigma_{\diamond}^{\prime}=\rho_{a}(h) \cdot \sigma_{\diamond}+A_{\diamond} \widetilde{\Pi}_{2}-\widetilde{\Pi}_{1}^{\prime} M_{\diamond} .
$$

According to Lemma 6.3, we get

$$
h_{*}\left(e_{\diamond}\right)=h_{*}\left(\Phi_{\Pi_{1}, \Pi_{2}}\left(\sigma_{\diamond}\right)\right)=\Phi_{\Pi_{1}^{\prime}, \Pi_{2}}\left(\rho_{a}(h) \cdot \sigma_{\diamond}\right) .
$$

This completes the proof of (b).

Corollary 6.1. For $e \in \operatorname{Ext}\left(T_{2}, T_{1}\right)_{\mathrm{pt}}$ and $n \in \mathbb{Z}$, we have

$$
n_{\mathfrak{A}_{2}}^{*}(e)=n \cdot e=\left(n_{\mathfrak{A}_{1}}\right)_{*}(e) .
$$

Proof. We consider the following commutative diagram:

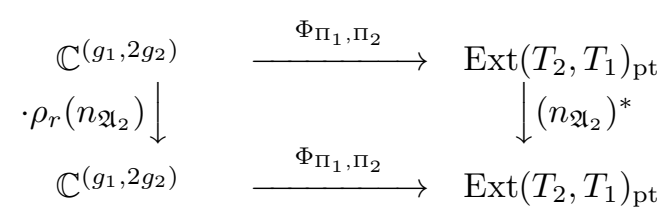

Since $\rho_{r}\left(n_{\mathfrak{A}_{2}}\right)=n I_{2 g_{2}}$, we get

$$
\left(n_{\mathfrak{A}_{2}}\right)^{*}(e)=\Phi_{\Pi_{1}, \Pi_{2}}(n \sigma)=n \cdot \Phi_{\Pi_{1}, \Pi_{2}}(\sigma)=n \cdot e .
$$

By a similar argument, we get

$$
\left(n_{\mathfrak{A}_{1}}\right)_{*}(e)=n \cdot e .
$$

Proposition 6.3. We have an isomorphism of abelian groups

$$
\mathbb{C}^{\left(g_{1}, g_{2}\right)} /\left(I_{g_{1}}, \Pi_{1}\right) \mathbb{Z}^{\left(2 g_{1}, 2 g_{2}\right)}\left(\begin{array}{l}
\Pi_{2} \\
I_{g_{2}}
\end{array}\right) \cong \operatorname{Ext}\left(T_{2}, T_{1}\right)_{\mathrm{pt}} .
$$

Proof. Let $\sigma=\left(\sigma_{1}, \sigma_{2}\right) \in \mathbb{C}^{\left(g_{1}, 2 g_{2}\right)}$ with $\sigma_{1}, \sigma_{2} \in \mathbb{C}^{\left(g_{1}, g_{2}\right)}$ corresponding to the extension $e=\Phi_{\Pi_{1}, \Pi_{2}}(\sigma) \in \operatorname{Ext}\left(T_{2}, T_{1}\right)_{\mathrm{pt}}$. By Lemma 6.3, the matrix

$$
\sigma-\sigma_{1} \widetilde{\Pi}_{2}=\left(\sigma_{1}, \sigma_{2}\right)-\sigma_{1}\left(I_{g_{2}}, \Pi_{2}\right)=\left(0, \sigma_{2}-\sigma_{1} \Pi_{2}\right)
$$


corresponds to the same extension $e$. This shows that every extension in $\operatorname{Ext}\left(T_{2}, T_{1}\right)_{\mathrm{pt}}$ can be represented by a matrix $\sigma=(0, \alpha)$ with $\alpha \in \mathbb{C}^{\left(g_{1}, g_{2}\right)}$. Hence we get a surjective homomorphism of abelian groups

$$
\mathbb{C}^{\left(g_{1}, g_{2}\right)} \longrightarrow \operatorname{Ext}\left(T_{2}, T_{1}\right)_{\mathrm{pt}}
$$

According to Lemma 6.3 , the matrices $\alpha$ and $\alpha^{\prime} \in \mathbb{C}^{\left(g_{1}, g_{2}\right)}$ define the same extension if and only if

$$
\left(0, \alpha-\alpha^{\prime}\right)=\widetilde{\Pi}_{1}\left(\begin{array}{ll}
M_{1} & M_{2} \\
M_{3} & M_{4}
\end{array}\right)+A \widetilde{\Pi}_{2}
$$

with $\left(\begin{array}{ll}M_{1} & M_{2} \\ M_{3} & M_{4}\end{array}\right) \in \mathbb{Z}^{\left(2 g_{1}, 2 g_{2}\right)}$ and $A \in \mathbb{C}^{\left(g_{1}, g_{2}\right)}$. From the equation (6.17) we get

$$
A=-M_{1}-\Pi_{1} M_{3} \text {. }
$$

Thus we have

$$
\begin{aligned}
\alpha-\alpha^{\prime} & =\Pi_{1} M_{4}-\Pi_{1} M_{3} \Pi_{2}+M_{2}-M_{1} \Pi_{2} \\
& =\left(I_{g_{1}}, \Pi_{1}\right)\left(\begin{array}{ll}
-M_{1} & M_{2} \\
-M_{3} & M_{4}
\end{array}\right)\left(\begin{array}{c}
\Pi_{2} \\
I_{g_{2}}
\end{array}\right) .
\end{aligned}
$$

This completes the proof of the above proposition.

\section{Line bundles over a polarized real torus}

Before we investigate complex line bundles over a real torus, we need a knowledge of holomorphic line bundles on a complex torus. We briefly review some results on holomorphic line bundles on a complex torus (cf. $[13,17,27$, $28]$ ).

Let $X=\mathbb{C}^{g} / L$ be a complex torus, where $L$ is a lattice in $\mathbb{C}^{g}$. The exponential sequence $0 \longrightarrow \mathbb{Z} \longrightarrow \mathcal{O}_{X} \longrightarrow \mathcal{O}_{X}^{*} \longrightarrow 1$ induces the long exact sequence

$$
\cdots \longrightarrow H^{1}(X, \mathbb{Z}) \longrightarrow H^{1}\left(X, \mathcal{O}_{X}\right) \longrightarrow H^{1}\left(X, \mathcal{O}_{X}^{*}\right) \stackrel{c_{1}}{\longrightarrow} H^{2}(X, \mathbb{Z}) \longrightarrow \cdots
$$

We recall that the Néron-Severi group $N S(X)$ (resp. $\left.P i c^{0}(X)\right)$ is defined to be the image of $c_{1}$ (resp. the kernel of $c_{1}$ ). For a hermitian form $H$ on $\mathbb{C}^{g}$ whose imaginary part $E_{H}:=\operatorname{Im}(H)$ is integral on $L \times L$, a semi-character for $H$ is defined to be a map $\alpha: L \longrightarrow \mathbb{C}_{1}^{*}$ is defined to be a map such that

$$
\alpha\left(\ell_{1}+\ell_{2}\right)=\alpha\left(\ell_{1}\right) \alpha\left(\ell_{2}\right) e^{\pi i E_{H}\left(\ell_{1}, \ell_{2}\right)}, \quad \ell_{1}, \ell_{2} \in L .
$$

We let $\operatorname{Her}(L)$ be the set of all hermitian forms on $\mathbb{C}^{g}$ whose imaginary parts are integral on $L \times L$. For any $H \in \operatorname{Her}(L)$, we denote by $\operatorname{SC}(H)$ the set of all semi-characters for $H$. To each pair $(H, \alpha)$ with $H \in \operatorname{Her}(L)$ and $\alpha \in \operatorname{SC}(H)$, we associate the automorphic factor $J_{H, \alpha}: L \times \mathbb{C}^{g} \longrightarrow \mathbb{C}^{*}$ defined by

$$
J_{H, \alpha}(\ell, z):=\alpha(\ell) e^{\frac{\pi}{2} H(\ell, \ell)+\pi H(z, \ell)}, \quad \ell \in L, z \in \mathbb{C}^{g} .
$$

A lattice $L$ acts on the trivial line bundle $\mathbb{C}^{g} \times \mathbb{C}$ on $\mathbb{C}^{g}$ freely by

$$
\ell \cdot(z, \xi)=\left(z+\ell, J_{H, \alpha}(\ell, z) \xi\right), \quad \ell \in L, z \in \mathbb{C}^{g}, \xi \in \mathbb{C} .
$$


The quotient

$$
\mathfrak{L}(H, \alpha):=\left(\mathbb{C}^{g} \times \mathbb{C}\right) / L
$$

obtained by the action (7.2) of $L$ has a natural structure of a holomorphic line bundle over $X$. We note that for each such pairs $\left(H_{1}, \alpha_{1}\right)$ and $\left(H_{2}, \alpha_{2}\right)$, we have

$J_{H_{1}, \alpha_{1}} \cdot J_{H_{2}, \alpha_{2}}=J_{H_{1}+H_{2}, \alpha_{1} \alpha_{2}}$ and $\mathfrak{L}\left(H_{1}, \alpha_{1}\right) \otimes \mathfrak{L}\left(H_{2}, \alpha_{2}\right)=\mathfrak{L}\left(H_{1}+H_{2}, \alpha_{1} \alpha_{2}\right)$.

Let $\mathfrak{B}(L)$ be the set of all pairs $(H, \alpha)$ with $H \in \operatorname{Her}(L)$ and $\alpha \in \operatorname{SC}(H)$. Then $\mathfrak{B}(L)$ has a group structure equipped with multiplication law

$\left(H_{1}, \alpha_{1}\right) \cdot\left(H_{2}, \alpha_{2}\right)=\left(H_{1}+H_{2}, \alpha_{1} \alpha_{2}\right), \quad H_{i} \in \operatorname{Her}(L), \alpha_{i} \in \operatorname{SC}\left(H_{i}\right), i=1,2$.

Appell-Humbert Theorem says that we have the following canonical isomorphism of exact sequences:

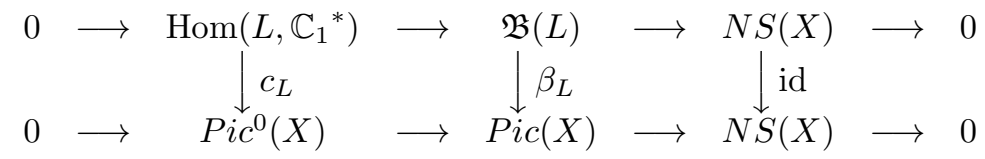

Here $\beta_{L}: \mathfrak{B}(L) \longrightarrow \operatorname{Pic}(X)=H^{1}\left(X, \mathcal{O}_{X}^{*}\right)$ is the group isomorphism defined by

$$
\beta_{L}((H, \alpha)):=\mathfrak{L}(H, \alpha), \quad(H, \alpha) \in \mathfrak{B}(L)
$$

and $c_{L}$ is the isomorphism induced by $\beta_{L}$. It is known that $N S(X)$ is a free abelian group of rank $\rho(X) \leq g^{2}$, where $\rho(X)$ is the Picard number of $X$. By Appell-Humbert Theorem, $N S(X)$ is realized in several ways as follows:

$$
\begin{aligned}
N S(X) & =\operatorname{Pic}(X) / \operatorname{Pic}^{0}(X)=c_{1}\left(H^{1}\left(X, \mathcal{O}_{X}^{*}\right)\right) \\
& =\left\{H: \mathbb{C}^{g} \times \mathbb{C}^{g} \rightarrow \mathbb{C} \text { hermitian, } \operatorname{Im}(H)(L \times L) \subseteq \mathbb{Z}\right\} \\
& =\left\{E: \mathbb{C}^{g} \times \mathbb{C}^{g} \rightarrow \mathbb{R} \text { alternating, } E(L \times L) \subseteq \mathbb{Z}, E(i \cdot, \cdot) \text { symmetric }\right\} .
\end{aligned}
$$

Let $\widehat{X}=P i c^{0}(X)$ be the dual complex torus of $X$. There exists the holomorphic line bundle $\mathscr{P}$ over $X \times \widehat{X}$ uniquely determined up to isomorphism, the so-called Poincaré bundle satisfying the following properties (PB1) and (PB2):

(PB1) $\left.\mathscr{P}\right|_{X \times L} \cong L$ for all $L \in \widehat{X}$, and

(PB2) $\left.\mathscr{P}\right|_{\{0\} \times \widehat{X}}$ is trivial on $\widehat{X}$.

We can see that $H^{g}(X, \mathscr{P})=\mathbb{C}$ and $H^{q}(X, \mathscr{P})=0$ for all $q \neq g$.

Let $T_{\Lambda}=V / \Lambda$ be a real torus of dimension $g$, where $V \cong \mathbb{R}^{g}$ is a real vector space of dimension $g$ and $\Lambda$ is a lattice in $V$. Let $\rho: \Lambda \longrightarrow \mathbb{C}^{*}$ be a character of $\Lambda$. Let $B: V \times V \longrightarrow \mathbb{R}$ be a real valued symmetric bilinear form on $V$. We define the map $I_{B, \rho}: \Lambda \times V \longrightarrow \mathbb{C}^{*}$ by

$$
I_{B, \rho}(\lambda, v)=\rho(\lambda) e^{\pi B(\lambda, \lambda)+2 \pi B(v, \lambda)}, \quad \lambda \in \Lambda, v \in V, \eta \in \mathbb{C} .
$$

It is easily checked that $I_{B, \rho}$ satisfies the following equation

$$
I_{B, \rho}\left(\lambda_{1}+\lambda_{2}, v\right)=I_{B, \rho}\left(\lambda_{1}, \lambda_{2}+v\right) I_{B, \rho}\left(\lambda_{2}, v\right), \quad \lambda_{1}, \lambda_{2} \in \Lambda, v \in V .
$$


Then $\Lambda$ acts on the trivial line bundle $V \times \mathbb{C}$ over $V$ freely by

$$
\lambda \cdot(v, \eta)=\left(v+\lambda, I_{B, \rho}(\lambda, v) \eta\right), \quad \lambda \in \Lambda, v \in V, \eta \in \mathbb{C} .
$$

Thus the quotient space

$$
L(B, \rho)=(V \times \mathbb{C}) / \Lambda
$$

has a natural structure of a smooth (or real analytic) line bundle over a real torus $T_{\Lambda}$.

Lemma 7.1. Suppose $B: V \times V \longrightarrow \mathbb{R}$ is a positive definite bilinear form on $V$. We define the function $\theta_{B, \rho}: V \longrightarrow \mathbb{C}$ by

$$
\theta_{B, \rho}(v)=\sum_{\lambda \in \Lambda} \rho(\lambda)^{-1} e^{-\pi B(\lambda, \lambda)-2 \pi B(v, \lambda)}, \quad v \in V .
$$

Then map $\Theta_{B, \rho}: V \longrightarrow V \times \mathbb{C}$ defined by

$$
\Theta_{B, \rho}(v)=\left(v, \theta_{B, \rho}(v)\right), \quad v \in V
$$

defines a smooth (or real analytic) global section of the line bundle $L(B, \rho)$.

Proof. For any $\lambda \in \Lambda$ and $v \in V$, we have

$$
\begin{aligned}
& \theta_{B, \rho}(\lambda+v) \\
= & \sum_{\mu \in \Lambda} \rho(\mu)^{-1} e^{-\pi B(\mu, \mu)-2 \pi B(\lambda+v, \mu)} \\
= & \rho(\lambda) e^{\pi B(\lambda, \lambda)+2 \pi B(v, \lambda)} \sum_{\mu \in \Lambda} \rho(\lambda+\mu)^{-1} e^{-\pi B(\lambda+\mu, \lambda+\mu)-2 \pi B(v, \lambda+\mu)} \\
= & I_{B, \rho}(\lambda, v) \theta_{B, \rho}(v) .
\end{aligned}
$$

Therefore $\Theta_{B, \rho}$ is a smooth global section of $L(B, \rho)$.

Lemma 7.2. Suppose $B: V \times V \longrightarrow \mathbb{R}$ is a positive definite bilinear form on $V$. Assume $B$ is integral on $\Lambda \times \Lambda$, that is, $B(\Lambda \times \Lambda) \subset \mathbb{Z}$. Then for any character $\rho: \Lambda \longrightarrow \mathbb{C}$, the function $f_{B, \rho}: V \longrightarrow \mathbb{C}$ defined by

$$
f_{B, \rho}(v)=\sum_{\lambda \in \Lambda} \rho(\lambda) e^{-\pi B(\lambda, \lambda)+2 \pi i B(v, \lambda)}, \quad v \in V
$$

is invariant under the action of $\Lambda$. Therefore $f_{B, \rho}$ may be regarded as a function on $T_{\Lambda}$.

Proof. It follows immediately from the definition.

We see that

$$
L_{\Lambda}=\mathbb{Z}^{g}+i \Lambda \subset \mathbb{C}^{g}
$$

is a lattice in $\mathbb{C}^{g}$. We consider the complex torus

$$
\mathfrak{T}_{\Lambda}=\mathbb{C}^{g} / L_{\Lambda}
$$

We define the $\mathbb{R}$-linear map $S_{B}: \mathbb{C}^{g} \times \mathbb{C}^{g} \longrightarrow \mathbb{R}$ and $E_{B}: \mathbb{C}^{g} \times \mathbb{C}^{g} \longrightarrow \mathbb{R}$ by

$$
S_{B}(x, y)=B\left(x_{1}, y_{1}\right)+B\left(x_{2}, y_{2}\right)
$$


and

$$
E_{B}(x, y)=B\left(x_{2}, y_{1}\right)-B\left(x_{1}, y_{2}\right),
$$

where $x=x_{1}+i x_{2} \in \mathbb{C}^{g}$ and $y=y_{1}+i y_{2} \in \mathbb{C}^{g}$ with $x_{1}, x_{2}, y_{1}, y_{2} \in \mathbb{R}^{g}$. It is easily seen that $S_{B}$ is symmetric and $E_{B}$ is alternating. We note that $S_{B}(x, y)=E_{B}(i x, y)$ for all $x, y \in \mathbb{C}^{g}$. We define the hermitian form $H_{B}$ : $\mathbb{C}^{g} \times \mathbb{C}^{g} \longrightarrow \mathbb{C}$ by

$$
H_{B}(x, y):=S_{B}(x, y)+i E_{B}(x, y), \quad x, y \in \mathbb{C}^{g} .
$$

Moreover we assume that $E_{B}$ is integral on $L_{\Lambda} \times L_{\Lambda}$. Let $\alpha: L_{\Lambda} \longrightarrow \mathbb{C}_{1}^{*}$ be a semi-character of $L_{\Lambda}$ for $H_{B}$ such that

$$
\alpha\left(\ell_{1}+\ell_{2}\right)=\alpha\left(\ell_{1}\right) \alpha\left(\ell_{2}\right) e^{\pi i E_{B}\left(\ell_{1}, \ell_{2}\right)}, \quad \ell_{1}, \ell_{2} \in L_{\Lambda} .
$$

Then the mapping $J_{B, \alpha}: L_{\Lambda} \times \mathbb{C}^{g} \longrightarrow \mathbb{C}^{*}$ defined by

$$
J_{B, \alpha}(\ell, z)=\alpha(\ell) e^{\frac{\pi}{2} H_{B}(\ell, \ell)+\pi H_{B}(z, \ell)}, \quad \ell \in L_{\Lambda}, \quad z \in \mathbb{C}^{g}
$$

is an automorphic factor for $L_{\Lambda}$ on $\mathbb{C}^{g}$. Clearly $L_{\Lambda}$ acts on the trivial line bundle $\mathbb{C}^{g} \times \mathbb{C}$ over $\mathbb{C}^{g}$ freely by

$$
\ell \cdot(z, \xi)=\left(\ell+z, J_{B, \alpha}(\ell, z) \xi\right), \quad \ell \in L_{\Lambda}, z \in \mathbb{C}^{g}, \xi \in \mathbb{C} .
$$

The quotient

$$
\mathfrak{L}(B, \alpha):=\left(\mathbb{C}^{g} \times \mathbb{C}\right) / L_{\Lambda}
$$

of $\mathbb{C}^{g} \times \mathbb{C}$ by $L_{\Lambda}$ has a natural structure of a holomorphic line bundle over a complex torus $\mathfrak{T}_{\Lambda}$.

In summary, to each pair $(B, \alpha)$ with s symmetric $\mathbb{R}$-bilinear form $B$ on $V$ such that $E_{B}$ is integral on $L_{\Lambda} \times L_{\Lambda}$ and a semi-character $\alpha$ for $H_{B}$ there is associated the holomorphic line bundle $\mathfrak{L}(B, \alpha)$ over $\mathfrak{T}_{\Lambda}$.

We assume that $B$ is non-degenerate of signature $(r, s)$ with $r+s=g$. Then the hermitian form $H_{B}$ is also non-degenerate of signature $(r, s)$. Moreover we assume that $E_{B}$ is integral on $L_{\Lambda} \times L_{\Lambda}$. Under these assumptions, Matsushima [15] proved that the cohomology group $H^{q}\left(\mathfrak{T}_{\Lambda}, \mathfrak{L}(B, \alpha)\right)=0$ for all $q \neq s$ and that $H^{s}\left(\mathfrak{T}_{\Lambda}, \mathfrak{L}(B, \alpha)\right)$ is identified with the complex vector space of all $C^{\infty}$ functions $f$ on $\mathbb{C}^{g}$ satisfying the following conditions:

(a) $f$ is a differentiable theta functions for the automorphic factor $J_{B, \alpha}$; namely we have

$$
f(\ell+z)=J_{B, \alpha}(\ell, z) f(z), \quad \ell \in L_{\Lambda}, z \in \mathbb{C}^{g},
$$

(b) $\frac{\partial f}{\partial \bar{z}_{i}}=0$ for all $i \in\{1,2, \ldots, r\}$ and

$$
\frac{\partial f}{\partial z_{i}}+\pi \bar{z}_{i} f=0 \quad \text { for all } i \in\{r+1, \ldots, g\}
$$

where $\left(z_{1}, \ldots, z_{g}\right)$ is the coordinate of $\mathbb{C}^{g}$ determined by a privileged basis of $\mathbb{C}^{g}$ for the hermitian form $H_{B}$. We can show that the cohomology group $H^{s}\left(\mathfrak{T}_{\Lambda}, \mathfrak{L}(B, \alpha)^{\otimes 3}\right)$ defines a smooth embedding of $\mathfrak{T}_{\Lambda}$ into the projective space 
$\mathbb{P}^{d}(\mathbb{C})$ with $d+1=\operatorname{dim} H^{s}\left(\mathfrak{T}_{\Lambda}, \mathfrak{L}(B, \alpha)^{\otimes 3}\right)$ which is holomorphic in $z_{1}, \ldots, z_{r}$ and anti-holomorphic in $z_{r+1}, \ldots, z_{g}$ (cf. [15] and [17]).

We consider the canonical semi-character $\gamma_{\Lambda, B}$ of $L_{\Lambda}$ defined by

$$
\gamma_{\Lambda, B}(\kappa+i \lambda):=e^{\pi i E_{B}(\kappa, i \lambda)}, \quad \kappa \in \mathbb{Z}^{g}, \lambda \in \Lambda .
$$

Then $\gamma_{\Lambda, B}$ defines the holomorphic line bundle $\mathfrak{L}\left(B, \gamma_{\Lambda, B}\right)$ over a complex torus $\mathfrak{T}_{\Lambda}$. For any $z \in \mathfrak{T}_{\Lambda}$ we denote by $T_{z}$ the translation of $\mathfrak{T}_{\Lambda}$ by $z$. Let $\pi_{\Lambda}: \mathbb{C}^{g} \longrightarrow$ $\mathfrak{T}_{\Lambda}$ be the natural projection. Then there exists an element $c_{\Lambda, B, \alpha}$ of $\mathbb{C}^{g}$ such that

$$
\mathfrak{L}(B, \alpha)=T_{\pi_{\Lambda}\left(c_{\Lambda, B, \alpha}\right)}^{*} \mathfrak{L}\left(B, \gamma_{\Lambda, B}\right) .
$$

$c_{\Lambda, B, \alpha}$ is called a characteristic of the holomorphic line bundle $\mathfrak{L}(B, \alpha)$. We refer to [13] for detail.

Now we let $T_{\Lambda}=V / \Lambda$ be a polarized real torus of dimension $g$. Its associated polarized real abelian variety

$$
\mathfrak{A}_{\Lambda}=\mathbb{C}^{g} / L_{\Lambda}, \quad L_{\Lambda}=\mathbb{Z}^{g}+i \Lambda
$$

admits a positive definite hermitian form $H_{\Lambda}$ on $\mathbb{C}^{g}$ whose imaginary part $\operatorname{Im}\left(H_{\Lambda}\right)$ is integral on $\Lambda \times \Lambda$ (cf. [17, p. 35]). We write

$$
H_{\Lambda}(x, y)=S_{\Lambda}(x, y)+i E_{\Lambda}(x, y), \quad x, y \in \mathbb{C}^{g},
$$

where $S_{\Lambda}$ and $E_{\Lambda}$ are the real part (resp. imaginary part) of $H_{\Lambda}$ respectively. We know that $S_{\Lambda}$ is a real valued symmetric bilinear form on $V$ and $E_{\Lambda}$ is a real valued alternating bilinear form on $V$. Let $\alpha_{\Lambda}: L_{\Lambda} \longrightarrow \mathbb{C}_{1}^{*}$ be a canonical semi-character of $L_{\Lambda}$ defined by

$$
\alpha_{\Lambda}(\kappa+i \lambda):=e^{\pi i E_{\Lambda}(\kappa, i \lambda)}, \quad \kappa \in \mathbb{Z}^{g}, \lambda \in \Lambda .
$$

We let $J_{H_{\Lambda}, \alpha_{\Lambda}}: L_{\Lambda} \times \mathbb{C}^{g} \longrightarrow \mathbb{C}^{*}$ be the automorphic factor for $\Lambda$ on $V$ that is canonically given by

$$
J_{H_{\Lambda}, \alpha_{\Lambda}}(\ell, z)=\alpha_{\Lambda}(\ell) e^{\frac{\pi}{2} H_{\Lambda}(\ell, \ell)+\pi H_{\Lambda}(z, \ell)}, \quad \ell \in L_{\Lambda}, \quad z \in \mathbb{C}^{g} .
$$

Obviously $L_{\Lambda}$ acts on $\mathbb{C}^{g} \times \mathbb{C}$ freely by

$$
\ell \cdot(z, \xi)=\left(\ell+z, J_{H_{\Lambda}, \alpha_{\Lambda}}(\ell, z) \xi\right), \quad \ell \in L_{\Lambda}, z \in \mathbb{C}^{g}, \xi \in \mathbb{C} .
$$

So the quotient space

$$
\mathfrak{L}\left(H_{\Lambda}, \alpha_{\Lambda}\right):=\left(\mathbb{C}^{g} \times \mathbb{C}\right) / L_{\Lambda}
$$

has a natural structure of a holomorphic line bundle over an abelian variety $\mathfrak{A}_{\Lambda}$.

Now we define the map $\Phi_{\Lambda}: T_{\Lambda} \longrightarrow \mathfrak{A}_{\Lambda}$ by

$$
\Phi_{\Lambda}(v+\Lambda):=i v+L_{\Lambda}, \quad v \in \mathbb{R}^{g} .
$$

$\Phi_{\Lambda}$ is a well defined injective mapping. It is well known that

$$
H^{q}\left(\mathfrak{A}_{\Lambda}, \mathfrak{L}\left(H_{\Lambda}, \alpha_{\Lambda}\right)\right)=0
$$


for all $q \neq 0$ and that the space of global holomorphic sections of $\mathfrak{L}\left(H_{\Lambda}, \alpha_{\Lambda}\right)^{\otimes n}$ for any positive integer $n \geq 3$ give a holomorphic embedding of $\mathfrak{A}_{\Lambda}$ as a closed complex manifold in a projective complex manifold $\mathbb{P}^{d}(\mathbb{C})$ (cf. [17, pp. 29-33]). Therefore we have a differentiable embedding of $T_{\Lambda}$ into a complex projective space $\mathbb{P}^{d}(\mathbb{C})$ and hence into a real projective space $\mathbb{P}^{N}(\mathbb{R})$ with large enough $N>0$.

We will characterize the pullback $L\left(\alpha_{\Lambda}\right):=\Phi_{\Lambda}^{*} \mathfrak{L}\left(H_{\Lambda}, \alpha_{\Lambda}\right)$. We first define the automorphic factor $I_{\alpha_{\Lambda}}: \Lambda \times \mathbb{R}^{g} \longrightarrow \mathbb{C}^{*}$ by

$$
I_{\alpha_{\Lambda}}(\lambda, v):=\alpha_{\Lambda}(i \lambda) e^{\frac{\pi}{2} H_{\Lambda}(\lambda, \lambda)+\pi H_{\Lambda}(v, \lambda)}, \quad \lambda \in \Lambda, v \in \mathbb{R}^{g} .
$$

This automorphic factor $I_{\alpha_{\Lambda}}$ yields the smooth (or real analytic) line bundle over $T_{\Lambda}$ which is nothing but the pullback $L\left(\alpha_{\Lambda}\right)$. We observe that if $\theta$ is a holomorphic theta function for $\mathfrak{L}\left(H_{\Lambda}, \alpha_{\Lambda}\right)$, then the function $f_{\theta}: \mathbb{R}^{g} \longrightarrow \mathbb{C}$ defined by $f_{\theta}(v):=\theta(i v), v \in \mathbb{R}^{g}$ defines a global smooth (or real analytic) section of $L\left(\alpha_{\Lambda}\right)$.

Now we will show that a holomorphic line bundle $\mathfrak{L}\left(H_{\Lambda}, \alpha_{\Lambda}\right)$ over $\mathfrak{A}_{\Lambda}$ naturally yields a smooth line bundle over a polarized torus $T_{\Lambda}$. Let $B_{\Lambda}$ be the restriction of $S_{\Lambda}$ to $\mathbb{R}^{g} \times \mathbb{R}^{g}$. First we define the automorphic factor $I_{B_{\Lambda}, \alpha_{\Lambda}}$ : $\Lambda \times \mathbb{R}^{g} \longrightarrow \mathbb{C}^{*}$ by

$$
I_{B_{\Lambda}, \alpha_{\Lambda}}(\lambda, v):=\alpha_{\Lambda}(2 i \lambda) e^{\pi B_{\Lambda}(\lambda, \lambda)+2 \pi B_{\Lambda}(v, \lambda)}, \quad \lambda \in \Lambda, v \in \mathbb{R}^{g} .
$$

This automorphic factor $I_{B_{\Lambda}, \alpha_{\Lambda}}(\lambda, v)$ yields a smooth line bundle

$$
L\left(B_{\Lambda}, \alpha_{\Lambda}\right):=\left(\mathbb{R}^{g} \times \mathbb{C}\right) / \Lambda
$$

over a polarized real torus $T_{\Lambda}$. Since $B_{\Lambda}$ is positive definite, according to Lemma 4.1, the space $\Gamma\left(T_{\Lambda}, L\left(B_{\Lambda}, \alpha_{\Lambda}\right)\right)$ of smooth (or real analytic) global sections of $L\left(B_{\Lambda}, \alpha_{\Lambda}\right)$ is not zero. If $B_{\Lambda}$ is integral on $\Lambda \times \Lambda$, according to Lemma 4.2, we see that the function $f_{\Lambda, \alpha_{\Lambda}}: \mathbb{R}^{g} \longrightarrow \mathbb{C}$ defined by

$$
f_{\Lambda, \alpha_{\Lambda}}(v)=\sum_{\lambda \in \Lambda} \alpha_{\Lambda}(2 i \lambda) e^{-\pi B_{\Lambda}(\lambda, \lambda)+2 \pi i B_{\Lambda}(v, \lambda)}, \quad v \in \mathbb{R}^{g}
$$

is a function on $T_{\Lambda}$.

So far we have proved the following.

Theorem 7.1. Let $T_{\Lambda}=V / \Lambda$ be a polarized real torus of dimension $g$. Then there is a smooth line bundle $L\left(B_{\Lambda}, \alpha_{\Lambda}\right)$ over $T_{\Lambda}$ which is constructed canonically by (7.23).

Example 7.1. Let $Y \in \mathcal{P}_{g}$ be a $g \times g$ positive definite symmetric real matrix. Then $\Lambda_{Y}=Y \mathbb{Z}^{g}$ is a lattice in $\mathbb{R}^{g}$. Then the $g$-dimensional torus $T_{Y}=\mathbb{R}^{g} / \Lambda_{Y}$ is a principally polarized real torus. Indeed,

$$
\mathfrak{A}_{Y}=\mathbb{C}^{g} / L_{Y}, \quad L_{Y}=\mathbb{Z}^{g}+i \Lambda_{Y}
$$

is a princially polarized real abelian variety (cf. Example 6.1). Its corresponding hermitian form $H_{Y}$ is given by

$$
H_{Y}(x, y)=S_{Y}(x, y)+i E_{Y}(x, y)={ }^{t} x Y^{-1} \bar{y}, \quad x, y \in \mathbb{C}^{g},
$$


where $S_{Y}$ and $E_{Y}$ denote the real part and the imaginary part of $H_{Y}$ respectively. Let $\alpha: L_{Y} \longrightarrow \mathbb{C}_{1}^{*}$ be a semi-character of $L_{Y}$. To a pair $\left(H_{Y}, \alpha\right)$ the canonical automorphic factor $J_{Y, \alpha}: L_{Y} \times \mathbb{C}^{g} \longrightarrow \mathbb{C}$ is associated by

$$
J_{Y, \alpha}(\ell, z)=\alpha(\ell) e^{\frac{1}{2} \pi i^{t} \ell Y^{-1} \bar{\ell}+\pi i^{t} z Y^{-1} \bar{\ell}}, \quad \ell \in L_{Y}, z \in \mathbb{C}^{g} .
$$

The associated automorphic factor $I_{Y, \alpha}: \Lambda_{Y} \times \mathbb{R}^{g} \longrightarrow \mathbb{C}^{*}$ is given by

$$
I_{Y, \alpha}(\lambda, v)=\alpha(2 i \lambda) e^{\pi^{t} \lambda Y^{-1} \lambda+2 \pi^{t} v Y^{-1} \lambda}, \quad \lambda \in \Lambda_{Y}, v \in \mathbb{R}^{g} .
$$

We get the associated line bundle

$$
L\left(B_{Y}, \alpha\right)=\left(\mathbb{R}^{g} \times \mathbb{C}\right) / \Lambda_{Y}
$$

given by $I_{Y, \alpha}$, where $B_{Y}$ is the restriction of $S_{Y}$ to $\mathbb{R}^{g} \times \mathbb{R}^{g}$. Then the function $\theta_{Y, \alpha}: \mathbb{R}^{g} \longrightarrow \mathbb{C}$ defined by

$$
\theta_{Y, \alpha}(v)=\sum_{\lambda \in \Lambda_{Y}} \alpha(2 i \lambda) e^{-\pi^{t} \lambda Y^{-1} \lambda-2 \pi^{t} v Y^{-1} \lambda}, \quad v \in \mathbb{R}^{g}
$$

yields a smooth global section of $L\left(B_{Y}, \alpha\right)$ over a real torus $T_{Y}$. The canonical semi-character $\alpha_{Y}$ of $L_{Y}$ is given by

$$
\alpha_{Y}(\kappa+i \lambda)=e^{-\pi i^{t} \kappa Y^{-1} \lambda}, \quad \kappa \in \mathbb{Z}^{g}, \lambda \in \Lambda_{Y} .
$$

\section{Moduli space for principally polarized real tori}

We have the natural action of $G L(g, \mathbb{R})$ on $\mathcal{P}_{g}$ given by

$$
A * Y=A Y^{t} A, \quad A \in G L(g, \mathbb{R}), Y \in \mathcal{P}_{g} .
$$

We put $\mathfrak{G}_{g}=G L(g, \mathbb{Z})$ (see Notations in the introduction). The fundamental domain $\mathfrak{R}_{g}$ for $\mathcal{P}_{g}$ with respect to $\mathfrak{G}_{g}$ which was found by H. Minkowski [16] is defined as a subset of $\mathcal{P}_{g}$ consisting of $Y=\left(y_{i j}\right) \in \mathcal{P}_{g}$ satisfying the following conditions (M.1)-(M.2) (cf. [10, p. 191] or [14, p. 123]):

(M.1) $a Y^{t} a \geq y_{k k}$ for every $a=\left(a_{i}\right) \in \mathbb{Z}^{g}$ in which $a_{k}, \ldots, a_{g}$ are relatively prime for $k=1,2, \ldots, g$.

(M.2) $y_{k, k+1} \geq 0$ for $k=1, \ldots, g-1$.

We say that a point of $\mathfrak{R}_{g}$ is Minkowski reduced or simply M-reduced. $\mathfrak{R}_{g}$ has the following properties (R1)-(R6):

(R1) For any $Y \in \mathcal{P}_{g}$, there exist a matrix $A \in G L(g, \mathbb{Z})$ and $R \in \mathfrak{R}_{g}$ such that $Y=R[A]$ (cf. [10, p. 191] or [14, p. 139]). That is,

$$
G L(g, \mathbb{Z}) \circ \mathfrak{R}_{g}=\mathcal{P}_{g} .
$$

(R2) $\mathfrak{R}_{g}$ is a convex cone through the origin bounded by a finite number of hyperplanes. $\mathfrak{R}_{g}$ is closed in $\mathcal{P}_{g}$ (cf. [14, p. 139]).

(R3) If $Y$ and $Y[A]$ lie in $\mathfrak{R}_{g}$ for $A \in G L(g, \mathbb{Z})$ with $A \neq \pm I_{g}$, then $Y$ lies on the boundary $\partial \mathfrak{R}_{g}$ of $\mathfrak{R}_{g}$. Moreover $\mathfrak{R}_{g} \cap\left(\mathfrak{R}_{g}[A]\right) \neq \emptyset$ for only finitely many $A \in G L(g, \mathbb{Z})$ (cf. [14, p. 139]). 
(R4) If $Y=\left(y_{i j}\right)$ is an element of $\mathfrak{R}_{g}$, then

$$
y_{11} \leq y_{22} \leq \cdots \leq y_{g g} \quad \text { and } \quad\left|y_{i j}\right|<\frac{1}{2} y_{i i} \quad \text { for } 1 \leq i<j \leq g .
$$

We refer to [10, p. 192] or [14, pp. 123-124].

For $Y=\left(y_{i j}\right) \in \mathcal{P}_{g}$, we put

$$
d Y=\left(d y_{i j}\right) \text { and } \frac{\partial}{\partial Y}=\left(\frac{1+\delta_{i j}}{2} \frac{\partial}{\partial y_{i j}}\right) .
$$

For a fixed element $A \in G L(g, \mathbb{R})$, we put

$$
Y_{*}=A \star Y=A Y^{t} A, \quad Y \in \mathcal{P}_{g} .
$$

Then

$$
d Y_{*}=A d Y^{t} A \quad \text { and } \quad \frac{\partial}{\partial Y_{*}}={ }^{t} A^{-1} \frac{\partial}{\partial Y} A^{-1} .
$$

We consider the following differential operators

$$
D_{k}=\sigma\left(\left(Y \frac{\partial}{\partial Y}\right)^{k}\right), \quad k=1,2, \ldots, g,
$$

where $\sigma(M)$ denotes the trace of a square matrix $M$. By Formula (8.2), we get

$$
\left(Y_{*} \frac{\partial}{\partial Y_{*}}\right)^{i}=A\left(Y \frac{\partial}{\partial Y}\right)^{i} A^{-1}
$$

for any $A \in G L(g, \mathbb{R})$. So each $D_{i}$ is invariant under the action (8.1) of $G L(g, \mathbb{R})$.

Selberg [20] proved the following.

Theorem 8.1. The algebra $\mathbb{D}\left(\mathcal{P}_{g}\right)$ of all differential operators on $\mathcal{P}_{g}$ invariant under the action $(8.1)$ of $G L(g, \mathbb{R})$ is generated by $D_{1}, D_{2}, \ldots, D_{g}$. Furthermore $D_{1}, D_{2}, \ldots, D_{g}$ are algebraically independent and $\mathbb{D}\left(\mathcal{P}_{g}\right)$ is isomorphic to the commutative ring $\mathbb{C}\left[x_{1}, x_{2}, \ldots, x_{g}\right]$ with $g$ indeterminates $x_{1}, x_{2}, \ldots, x_{g}$.

Proof. The proof can be found in [14, pp. 64-66].

We can see easily that

$$
d s^{2}=\sigma\left(\left(Y^{-1} d Y\right)^{2}\right)
$$

is a $G L(g, \mathbb{R})$-invariant Riemannian metric on $\mathcal{P}_{g}$ and its Laplacian is given by

$$
\Delta=\sigma\left(\left(Y \frac{\partial}{\partial Y}\right)^{2}\right)
$$

We also can see that

$$
d \mu_{g}(Y)=(\operatorname{det} Y)^{-\frac{g+1}{2}} \prod_{i \leq j} d y_{i j}
$$


is a $G L(g, \mathbb{R})$-invariant volume element on $\mathcal{P}_{g}$. The metric $d s^{2}$ on $\mathcal{P}_{g}$ induces the metric $d s_{\mathcal{R}}^{2}$ on $\mathfrak{R}_{g}$. Minkowski [16] calculated the volume of $\mathfrak{R}_{g}$ explicitly.

$\mathcal{P}_{g}$ parameterizes principally polarized real tori of dimension $g$. The Minkowski modular space $\mathfrak{T}_{g}$ is the moduli space for isomorphism classes of principally polarized real tori of dimension $g$. According to $(\mathrm{R} 2)$ we see that $\mathfrak{T}_{g}$ is a semialgebraic set with real analytic structure. Unfortunately $\mathfrak{T}_{g}$ does not admit the structure of a real algebraic variety and does not admit a compactification which is defined over the rational number field $\mathbb{Q}$. We see that $\mathfrak{T}_{g}$ is real analytically isomorphic to the semi-algebraic subset $\mathscr{X}_{(0,1)}^{g}$ of $\mathscr{X}_{\mathbb{R}}^{g}$. We define the embedding $\Phi_{g}: \mathcal{P}_{g} \longrightarrow \mathscr{H}_{g}$ by

$$
\Phi_{g}(Y)=i Y, \quad Y \in \mathcal{P}_{g} .
$$

We have the following inclusions

$$
\mathcal{P}_{g} \stackrel{\Phi_{g}}{\longrightarrow} i \mathcal{P}_{g} \hookrightarrow \mathscr{H}_{g} \hookrightarrow \mathbb{H}_{g} \subset \mathbb{H}_{g}^{*}
$$

$\mathfrak{G}_{g}$ acts on $\mathcal{P}_{g}$ and $i \mathcal{P}_{g}, \Gamma_{g}^{\star}$ acts on $\mathscr{H}_{g}$, and $\Gamma_{g}$ acts on $\mathbb{H}_{g}$ and $\mathbb{H}_{g}^{*}$. It might be interesting to characterize the boundary points of the closure of $i \mathcal{P}_{g}$ (or $\left.\mathcal{P}_{g}\right)$ in $\mathbb{H}_{g}^{*}$ explicitly. In Section 5 we reviewed Silhol's compactification $\frac{\mathscr{X}_{\mathbb{R}}^{g}}{\text { of }}$ $\mathscr{X}_{\mathbb{R}}^{g}$ which is analogous to the Satake-Baily-Borel compactification. The theory of automorphic forms on $\mathcal{P}_{g}$ for $G L(g, \mathbb{Z})$ has been developed by Selberg [20], Maass [14] et al. past a half century. According to Theorem 5.1, $\overline{\mathscr{X}_{\mathbb{R}}^{g}}$ is a connected compact Hausdorff space containing $\mathscr{X}_{\mathbb{R}}^{g}$ as an open dense subset of $\overline{\mathscr{X}_{\mathbb{R}}^{g}}$. But $\overline{\mathscr{X}_{\mathbb{R}}^{g}}$ does not admit an algebraic structure.

For any positive integer $h \in \mathbb{Z}^{+}$, we let

$$
G L_{g, h}:=G L(g, \mathbb{R}) \ltimes \mathbb{R}^{(h, g)}
$$

be the semi-direct product of $G L(g, \mathbb{R})$ and $\mathbb{R}^{(h, g)}$ with the multiplication law

$$
(A, a) \cdot(B, b)=\left(A B, a^{t} B^{-1}+b\right), \quad A, B \in G L(g, \mathbb{R}), \quad a, b \in \mathbb{R}^{(h, g)} .
$$

Then we have the natural action of $G L_{g, h}$ on the Minkowski-Euclid space $\mathcal{P}_{g} \times$ $\mathbb{R}^{(h, g)}$ defined by

(8.7) $(A, a) \cdot(Y, \zeta)=\left(A Y^{t} A,(\zeta+a){ }^{t} A\right),(A, a) \in G L_{g, h}, Y \in \mathcal{P}_{g}, \zeta \in \mathbb{R}^{(h, g)}$.

For a variable $(Y, V) \in \mathcal{P}_{g} \times \mathbb{R}^{(h, g)}$ with $Y \in \mathcal{P}_{g}$ and $V \in \mathbb{R}^{(h, g)}$, we put

$$
\begin{gathered}
Y=\left(y_{\mu \nu}\right) \text { with } y_{\mu \nu}=y_{\nu \mu}, \quad V=\left(v_{k l}\right), \\
d Y=\left(d y_{\mu \nu}\right), \quad d V=\left(d v_{k l}\right), \\
{[d Y]=\prod_{\mu \leq \nu} d y_{\mu \nu}, \quad[d V]=\prod_{k, l} d v_{k l},}
\end{gathered}
$$

and

$$
\frac{\partial}{\partial Y}=\left(\frac{1+\delta_{\mu \nu}}{2} \frac{\partial}{\partial y_{\mu \nu}}\right), \quad \frac{\partial}{\partial V}=\left(\frac{\partial}{\partial v_{k l}}\right),
$$

where $1 \leq \mu, \nu, l \leq g$ and $1 \leq k \leq h$. 
Lemma 8.1. For all two positive real numbers $A$ and $B$, the following metric $d s_{g, h ; A, B}^{2}$ on $\mathcal{P}_{g} \times \mathbb{R}^{(h, g)}$ defined by

$$
d s_{g, h ; A, B}^{2}=A \sigma\left(Y^{-1} d Y Y^{-1} d Y\right)+B \sigma\left(Y^{-1 t}(d V) d V\right)
$$

is a Riemannian metric on $\mathcal{P}_{g} \times \mathbb{R}^{(h, g)}$ which is invariant under the action (8.7) of $G L_{g, h}$. The Laplacian $\Delta_{g, h ; A, B}$ of $\left(\mathcal{P}_{g} \times \mathbb{R}^{(h, g)}, d s_{g, h ; A, B}^{2}\right)$ is given by $\Delta_{g, h ; A, B}=\frac{1}{A} \sigma\left(\left(Y \frac{\partial}{\partial Y}\right)^{2}\right)-\frac{h}{2 A} \sigma\left(Y \frac{\partial}{\partial Y}\right)+\frac{1}{B} \sum_{k \leq p}\left(\left(\frac{\partial}{\partial V}\right) Y^{t}\left(\frac{\partial}{\partial V}\right)\right)_{k p}$.

Moreover $\Delta_{g, h ; A, B}$ is a differential operator of order 2 which is invariant under the action (8.7) of $G L_{g, h}$.

Proof. For a fixed element $(A, a) \in G L_{g, h}$, we set

$$
\left(Y^{*}, V^{*}\right)=(A, a) \cdot(Y, V) .
$$

Then

$$
Y^{*}=A Y^{t} A, \quad V^{*}=(V+a)^{t} A .
$$

The first statement follows immediately from the fact that

$$
d Y^{*}=A d Y^{t} A \text { and } \quad d V^{*}=d V^{t} A .
$$

Using the formula (13) in [9, p. 245], we can compute the Laplacian $\Delta_{g, h ; A, B}$ of $\left(\mathcal{P}_{g} \times \mathbb{R}^{(h, g)}, d s_{g, h ; A, B}^{2}\right)$. The last statement follows from the fact that

$$
\frac{\partial}{\partial Y^{*}}={ }^{t} A^{-1} \frac{\partial}{\partial Y} A^{-1}, \quad \frac{\partial}{\partial V^{*}}=\frac{\partial}{\partial V} \cdot A^{-1} .
$$

Lemma 8.2. The following volume element $d v_{g, h}(Y, V)$ on $\mathcal{P}_{g} \times \mathbb{R}^{(h, g)}$ defined by

$$
d v_{g, h}(Y, V)=(\operatorname{det} Y)^{-\frac{g+h+1}{2}}[d Y][d V]
$$

is invariant under the action (8.7) of $G L_{g, h}$.

Proof. For a fixed element $(A, a) \in G L_{g, h}$, we set

$$
\left(Y^{*}, V^{*}\right)=(A, a) \cdot(Y, V)=\left(A Y^{t} A,(V+a)^{t} A\right) .
$$

Let $\frac{\partial\left(Y^{*}, V^{*}\right)}{\partial(Y, V)}$ be the Jacobian determinant of the action (8.7) of $G L_{g, h}$ on $\mathcal{P}_{g} \times \mathbb{R}^{(h, g)}$. It is known that the Jacobian determinant of the action $Y \longmapsto Y^{*}$ is given by

$$
\frac{\partial\left(Y^{*}\right)}{\partial(Y)}=(\operatorname{det} A)^{g+1}
$$

Take the diagonal matrix $g=\left(d_{1}, \ldots, d_{g}\right)$ with distinct real numbers $d_{i}$. Obviously if $a=\left(a_{k l}\right), V=\left(v_{k l}\right)$ and $V^{*}=\left(v_{k l}^{*}\right)$, then $v_{k l}^{*}=\left(v_{k l}+a_{k l}\right) d_{l}$ for all $k, l$. Thus we have

$$
\frac{\partial\left(V^{*}\right)}{\partial(V)}=\left(d_{1} \cdots d_{g}\right)^{h}=(\operatorname{det} A)^{h}
$$


Since the set of all $g \times g$ real matrices whose eigenvalues are all distinct is everywhere dense in $G L(g, \mathbb{R})$, and $\frac{\partial\left(V^{*}\right)}{\partial(V)}$ is a rational function, the relation (8.10) holds for any $A \in G L(g, \mathbb{R})$. It is easy to see that

$$
\frac{\partial\left(Y^{*}, V^{*}\right)}{\partial(Y, V)}=\frac{\partial\left(Y^{*}\right)}{\partial(Y)} \cdot \frac{\partial\left(V^{*}\right)}{\partial(V)} \text {. }
$$

Thus we obtain

$$
\left[d Y^{*}\right]\left[d V^{*}\right]=|\operatorname{det} A|^{g+h+1}[d Y][d V] .
$$

Since $\operatorname{det} Y^{*}=(\operatorname{det} A)^{2} \operatorname{det} Y$, we have

$$
\left(\operatorname{det} Y^{*}\right)^{-\frac{g+h+1}{2}}\left[d Y^{*}\right]\left[d V^{*}\right]=(\operatorname{det} Y)^{-\frac{g+h+1}{2}}[d Y][d V] .
$$

Hence the volume element (8.9) is invariant under the action (8.7).

It is known that

$$
d \mu_{g}(Y):=(\operatorname{det} Y)^{-\frac{g+1}{2}}[d Y]
$$

is a volume element on $\mathcal{P}_{g}$ invariant under the action $(8.1)$ of $G L(g, \mathbb{R})$ (cf. $[14$, p. 23]). Let $r$ be a positive integer with $0<r<g$. We define a bijective transformation

$$
\mathcal{P}_{g} \longrightarrow \mathcal{P}_{r} \times \mathcal{P}_{s} \times \mathbb{R}^{(s, r)}, \quad r+s=g, \quad Y \longmapsto(F, G, H)
$$

by

(8.11) $Y=\left(\begin{array}{cc}F & 0 \\ 0 & G\end{array}\right)\left[\left(\begin{array}{cc}I_{r} & 0 \\ H & I_{s}\end{array}\right)\right], \quad Y \in \mathcal{P}_{n}, F \in \mathcal{P}_{r}, G \in \mathcal{P}_{s}, H \in \mathbb{R}^{(s, r)}$.

According to [14, pp. 24-26], we obtain

$$
[d Y]=(\operatorname{det} G)^{r}[d F][d H][d G],
$$

equivalently

$$
d \mu_{g}(Y)=(\operatorname{det} F)^{-\frac{s}{2}}(\operatorname{det} G)^{\frac{r}{2}} d \mu_{r}(F) d \mu_{s}(G)[d H]
$$

Therefore we get

$$
d v_{g, h}(Y, V)=(\operatorname{det} F)^{-\frac{h+s}{2}}(\operatorname{det} G)^{\frac{r-h}{2}} d \mu_{r}(F) d \mu_{s}(G)[d H][d V] .
$$

Similarly if $Y \in \mathcal{P}_{g}, g=r+s$ with $0<r<g$, we write

$$
Y=\left(\begin{array}{cc}
P & 0 \\
0 & Q
\end{array}\right)\left[\left(\begin{array}{cc}
I_{r} & R \\
0 & I_{s}
\end{array}\right)\right], \quad Y \in \mathcal{P}_{g}, P \in \mathcal{P}_{r}, Q \in \mathcal{P}_{s}, R \in \mathbb{R}^{(r, s)}
$$

According to $[14$, p. 27], we obtain

$$
[d Y]=(\operatorname{det} P)^{s}[d P][d Q][d R],
$$

equivalently

$$
d \mu_{g}(Y)=(\operatorname{det} P)^{\frac{s}{2}}(\operatorname{det} G)^{-\frac{r}{2}} d \mu_{r}(P) d \mu_{s}(Q)[d R] .
$$

Therefore we get

$$
d v_{g, h}(Y, V)=(\operatorname{det} P)^{\frac{s-h}{2}}(\operatorname{det} G)^{-\frac{r+h}{2}} d \mu_{r}(P) d \mu_{s}(Q)[d R][d V] .
$$


The coordinates $(F, G, H)$ or $(P, Q, R)$ are called the partial Iwasawa coordinates on $\mathcal{P}_{g}$.

Theorem 8.2. Any geodesic through the origin $\left(I_{g}, 0\right)$ is of the form

$$
\gamma(t)=\left(\lambda(2 t)[k], Z\left(\int_{0}^{t} \lambda(t-s) d s\right)[k]\right)
$$

where $k$ is a fixed element of $O(g), Z$ is a fixed $h \times g$ real matrix, $t$ is a real variable, $\lambda_{1}, \lambda_{2}, \ldots, \lambda_{g}$ are fixed real numbers but not all zero and

$$
\lambda(t):=\operatorname{diag}\left(e^{\lambda_{1} t}, \ldots, e^{\lambda_{g} t}\right) .
$$

Furthermore, the tangent vector $\gamma^{\prime}(0)$ of the geodesic $\gamma(t)$ at $\left(I_{g}, 0\right)$ is $(D[k], Z)$, where $D=\operatorname{diag}\left(2 \lambda_{1}, \ldots, 2 \lambda_{g}\right)$.

Proof. Let $W=(X, Z)$ be an element of $\mathfrak{p}$ with $X \neq 0$. Then the curve

$$
\alpha(t)=\exp t W=\left(e^{t X}, Z\left(\int_{0}^{t} e^{-s X} d s\right)\right), \quad t \in \mathbb{R}
$$

is a geodesic in $G L_{g, h}$ with $\alpha^{\prime}(0)=W$ passing through the identity of $G L_{g, h}$. Thus the curve

$$
\gamma(t)=\alpha(t) \cdot\left(I_{g}, 0\right)=\left(e^{2 t X}, Z\left(\int_{0}^{t} e^{-s X} d s\right) e^{t X}\right)
$$

is a geodesic in $\mathcal{P}_{g} \times \mathbb{R}^{(h, g)}$ passing through the origin $\left(I_{g}, 0\right)$. Since $X$ is a symmetric real matrix, there is a diagonal matrix $\Lambda=\operatorname{diag}\left(\lambda_{1}, \ldots, \lambda_{g}\right)$ with $\lambda_{1}, \ldots, \lambda_{g} \in \mathbb{R}$ such that

$$
X={ }^{t} k \Lambda k \quad \text { for some } k \in O(g),
$$

where $\lambda_{1}, \ldots, \lambda_{n}$ are real numbers and not all zero. Thus we may write

$$
\gamma(t)=\left(\left(\delta_{k l} e^{2 \lambda_{k} t}\right)[k], Z\left(\int_{0}^{t} e^{(t-s) \Lambda} d s\right)[k]\right) .
$$

Hence this completes the proof.

Theorem 8.3. Let $\left(Y_{0}, V_{0}\right)$ and $\left(Y_{1}, V_{1}\right)$ be two points in $\mathcal{P}_{g} \times \mathbb{R}^{(h, g)}$. Let $g$ be an element in $G L(g, \mathbb{R})$ such that $Y_{0}\left[{ }^{t} g\right]=I_{g}$ and $Y_{1}\left[{ }^{t} g\right]$ is diagonal. Then the length $s\left(\left(Y_{0}, V_{0}\right),\left(Y_{1}, V_{1}\right)\right)$ of the geodesic joining $\left(Y_{0}, V_{0}\right)$ and $\left(Y_{1}, V_{1}\right)$ for the $G L_{g, h}$-invariant Riemannian metric $d s_{g, h ; A, B}^{2}$ is given by

$$
s\left(\left(Y_{0}, V_{0}\right),\left(Y_{1}, V_{1}\right)\right)=A\left\{\sum_{j=1}^{g}\left(\ln t_{j}\right)^{2}\right\}^{1 / 2}+B \int_{0}^{1}\left(\sum_{j=1}^{g} \Delta_{j} e^{-\left(\ln t_{j}\right) t}\right)^{1 / 2} d t
$$

where $\Delta_{j}=\sum_{k=1}^{h} \widetilde{v}_{k j}^{2}(1 \leq j \leq g)$ with $\left(V_{1}-V_{0}\right)^{t} g=\left(\widetilde{v}_{k j}\right)$ and $t_{1}, \ldots, t_{g}$ denotes the zeros of $\operatorname{det}\left(t Y_{0}-Y_{1}\right)$. 
Proof. Without loss of generality we may assume that $\left(Y_{0}, V_{0}\right)=\left(I_{g}, 0\right)$ and $\left(Y_{1}, V_{1}\right)=(T, \widetilde{V})$ with $T=\operatorname{diag}\left(t_{1}, \ldots, t_{n}\right)$ diagonal because the element $\left(g,-V_{0}\right) \in G L_{g, h}$ can be regarded as an isometry of $\mathcal{P}_{g} \times \mathbb{R}^{(h, g)}$ for the Riemannian metric $d s_{g, h ; A, B}^{2}$ (cf. Lemma 8.1). Let $\gamma(t)=(\alpha(t), \beta(t))$ with $0 \leq$ $t \leq 1$ be the geodesic in $\mathcal{P}_{g} \times \mathbb{R}^{(h, g)}$ joining two points $\gamma(0)=\left(Y_{0}, V_{0}\right)$ and $\gamma(1)=\left(Y_{1}, V_{1}\right)$, where $\alpha(t)$ is the uniquely determined curve in $\mathcal{P}_{g}$ and $\beta(t)$ is the uniquely determined curve in $\mathbb{R}^{(h, g)}$.

We now use the partial Iwasawa coordinates in $\mathcal{P}_{g}$. Then if $Y \in \mathcal{P}_{g}$, we write for any positive integer $r$ with $0<r<g, r+s=g$,

$$
Y=\left(\begin{array}{cc}
F & 0 \\
0 & G
\end{array}\right)\left[\left(\begin{array}{cc}
I_{r} & 0 \\
H & I_{s}
\end{array}\right)\right], \quad F \in \mathcal{P}_{r}, G \in \mathcal{P}_{s}, H \in \mathbb{R}^{(h, g)} .
$$

For $V \in \mathbb{R}^{(h, g)}$, we write

$$
V=(R, S), \quad R \in \mathbb{R}^{(h, r)}, S \in \mathbb{R}^{(h, s)} .
$$

Now we express $d s_{g, h ; A, B}^{2}$ in terms of $F, G, H, R$ and $S$.

\section{Lemma 8.3.}

$$
\begin{aligned}
d s_{g, h ; A, B}^{2}= & A \cdot\left\{\sigma\left(\left(F^{-1} d F\right)^{2}\right)+\sigma\left(\left(G^{-1} d G\right)^{2}\right)+2 \sigma\left(F^{-1 t}(d H) G d H\right)\right\} \\
& +B \cdot\left\{\sigma\left(F^{-1}{ }^{t}(d R) d R\right)+\sigma\left(\left(G^{-1}+F^{-1}\left[{ }^{t} H\right]\right){ }^{t}(d S) d S\right)\right\} \\
& -2 B \cdot \sigma\left(F^{-1 t} H^{t}(d S) d R\right) .
\end{aligned}
$$

Proof. First we see that if $Y \in \mathcal{P}_{g}$, then

$$
\begin{gathered}
Y^{-1}=\left(\begin{array}{cc}
F^{-1} & 0 \\
0 & G^{-1}
\end{array}\right)\left[\left(\begin{array}{cc}
I_{r} & -{ }^{t} H \\
0 & I_{s}
\end{array}\right)\right]=\left(\begin{array}{cc}
F^{-1} & -F^{-1 t} H \\
-H F^{-1} & G^{-1}+F^{-1}\left[{ }^{t} H\right]
\end{array}\right), \\
d Y=\left(\begin{array}{cc}
d F+d G[H]+{ }^{t}(d H) \cdot G H+{ }^{t} H G \cdot d H & (d H) \cdot G+{ }^{t} H \cdot d G \\
d G \cdot H+G \cdot d H & d G
\end{array}\right)
\end{gathered}
$$

and $d V=(d R, d S)$.

For brevity, we put

and

$$
d Y \cdot Y^{-1}=\left(\begin{array}{ll}
L_{0} & L_{1} \\
L_{2} & L_{3}
\end{array}\right)
$$

$$
Y^{-1 t}(d V) d V=\left(\begin{array}{ll}
M_{0} & M_{1} \\
M_{2} & M_{3}
\end{array}\right) .
$$

Here $L_{0}, L_{1}, L_{2}$ and $L_{3}$ denote the $r \times r, r \times s, s \times r$ and $s \times s$ matrix valued differential one forms respectively, and $M_{0}, M_{1}, M_{2}$ and $M_{3}$ denote the $r \times r$, $r \times s, s \times r$ and $s \times s$ matrix valued differential two forms respectively.

By an easy computation, we get

$$
\begin{aligned}
& L_{0}=d F \cdot F^{-1}+{ }^{t} H G \cdot d H \cdot F^{-1} \\
& L_{1}=-d F \cdot F^{-1 t} H-{ }^{t} H G \cdot d H \cdot F^{-1 t} H+{ }^{t}(d H)+{ }^{t} H \cdot d G \cdot G^{-1}, \\
& L_{2}=G \cdot d H \cdot F^{-1}
\end{aligned}
$$




$$
\begin{aligned}
L_{3} & =d G \cdot G^{-1}-G \cdot d H \cdot F^{-1 t} H, \\
M_{0} & =F^{-1 t}(d R) d R-F^{-1}{ }^{t} H^{t}(d S) d R, \\
M_{3} & =-H F^{-1 t}(d R) d S+\left(G^{-1}+F^{-1}\left[{ }^{t} H\right]\right)^{t}(d S) d S .
\end{aligned}
$$

Therefore we have

$$
\begin{aligned}
d s_{g, h ; A, B}^{2}= & A \cdot \sigma\left(\left(d Y \cdot Y^{-1}\right)^{2}\right)+B \cdot \sigma\left(Y^{-1 t}(d V) d V\right) \\
=A & \cdot\left\{\sigma\left(L_{0}^{2}+L_{1} L_{2}\right)+\sigma\left(L_{2} L_{1}+L_{3}^{2}\right)\right\} \\
& +B \cdot\left\{\sigma\left(M_{0}\right)+\sigma\left(M_{3}\right)\right\} \\
= & A \cdot\left\{\sigma\left(\left(F^{-1} d F\right)^{2}\right)+\sigma\left(\left(G^{-1} d G\right)^{2}\right)+2 \sigma\left(F^{-1 t}(d H) G d H\right)\right\} \\
& +B \cdot\left\{\sigma\left(F^{-1}(d R) d R\right)+\sigma\left(\left(G^{-1}+F^{-1}\left[{ }^{t} H\right]\right)^{t}(d S) d S\right)\right\} \\
& -2 B \cdot \sigma\left(F^{-1 t} H^{t}(d S) d R\right) .
\end{aligned}
$$

Let $s\left(\left(Y_{0}, V_{0}\right),\left(Y_{1}, V_{1}\right)\right)$ be the length of the geodesic $\gamma(t)=(\alpha(t), \beta(t))$ with $0 \leq t \leq 1$. We put

$$
\alpha(t)=\left(\begin{array}{cc}
F(t) & 0 \\
0 & G(t)
\end{array}\right)\left[\left(\begin{array}{cc}
I_{r} & 0 \\
H(t) & I_{s}
\end{array}\right)\right], \quad \beta(t)=(R(t), S(t)), \quad 0 \leq t \leq 1,
$$

where $F(t), G(t), H(t), R(t)$ and $S(t)$ are the uniquely determined curves in $\mathcal{P}_{r}, \mathcal{P}_{s}, \mathbb{R}^{(s, r)}, \mathbb{R}^{(h, r)}$ and $\mathbb{R}^{(h, s)}$ respectively.

Then we have

$$
\begin{aligned}
& s\left(\left(Y_{0}, V_{0}\right),\left(Y_{1}, V_{1}\right)\right) \\
= & A \cdot \int_{0}^{1}\left\{\sigma\left(\left(F^{-1} \frac{d F}{d t}\right)^{2}\right)+\sigma\left(\left(G^{-1} \frac{d G}{d t}\right)^{2}\right)\right. \\
& \left.+2 \sigma\left(F^{-1}\left(\frac{d H}{d t}\right) G \frac{d H}{d t}\right)\right\}^{1 / 2} d t \\
& +B \cdot \int_{0}^{1}\left\{\sigma\left(\gamma(t)^{-1}\left(\frac{d V}{d t}\right) \frac{d V}{d t}\right)\right\}^{1 / 2} d t \\
\geq & A \cdot \int_{0}^{1}\left\{\sigma\left(\left(F^{-1} \frac{d F}{d t}\right)^{2}\right)+\sigma\left(\left(G^{-1} \frac{d G}{d t}\right)^{2}\right)\right\}^{1 / 2} d t \\
& +B \cdot \int_{0}^{1}\left\{\sigma\left(F^{-1}\left(\frac{d R}{d t}\right) \frac{d R}{d t}\right)+\sigma\left(G^{-1}\left(\frac{d S}{d t}\right) \frac{d S}{d t}\right)\right\}^{1 / 2} d t .
\end{aligned}
$$

The reason is that the quadratic form $\sigma\left(F^{-1} t(d H) G d H\right)$ is positive definite. Indeed, if $M, N \in G L(g, \mathbb{R})$ such that $F={ }^{t} M M$ and $G={ }^{t} N N$, then

$$
\sigma\left(F^{-1}{ }^{t}(d H) G d H\right)=\sigma\left({ }^{t} W W\right), \quad W:=N \cdot d H \cdot M^{-1} .
$$

If

$$
\sigma\left(F^{-1}\left(\frac{d H}{d t}\right) G \frac{d H}{d t}\right)=0,
$$


then $\frac{d H}{d t}=0$ and hence $H(t)$ is constant in the interval $[0,1]$. Since $H(0)=$ $0, H(t)=0(0 \leq t \leq 1)$.

Moreover the curve $\alpha(t)$ must be diagonal, that is,

$$
\alpha(t)=\left(\delta_{\mu \nu} e^{\chi_{\nu}(t)}\right), \quad \chi_{\nu}(0)=0, \chi_{\nu}(1)=\ln t_{\nu}, \quad 1 \leq \nu \leq g
$$

where $g_{\nu}(t)(1 \leq \nu \leq g)$ are continuously differentiable in $[0,1]$. Thus we have

$$
\frac{d \alpha}{d t}=\left(\delta_{\mu \nu} e^{\chi_{\nu}(t)} \frac{d \chi_{\nu}}{d t}\right)
$$

and hence

$$
\alpha(t)^{-1} \frac{d \alpha}{d t}=\left(\delta_{\mu \nu} \frac{d \chi_{\nu}}{d t}\right)
$$

Therefore we have

$$
\begin{aligned}
& \int_{0}^{1}\left\{\sigma\left(\left(\alpha(t)^{-1} \frac{d \alpha}{d t}\right)^{2}\right)\right\}^{1 / 2} d t \\
= & \int_{0}^{1}\left\{\sigma\left(\left(F^{-1} \frac{d F}{d t}\right)^{2}\right)+\sigma\left(\left(G^{-1} \frac{d G}{d t}\right)^{2}\right)\right\}^{1 / 2} d t \\
= & \int_{0}^{1}\left\{\sum_{j=1}^{n}\left(\frac{d \chi_{j}}{d t}\right)^{2}\right\}^{1 / 2} d t .
\end{aligned}
$$

The minimum value of $\sum_{j=1}^{g}\left(\frac{d \chi_{j}}{d t}\right)^{2}$ is obtained if the curve $\alpha(t)$ is the straight line, i.e., $\chi_{j}(t)=t \ln t_{j}(1 \leq j \leq g), 0 \leq t \leq 1$ in the $\left(\chi_{1}, \ldots, \chi_{g}\right)$-space. Thus we get

$$
\int_{0}^{1}\left\{\sigma\left(\left(\alpha(t)^{-1} \frac{d \alpha}{d t}\right)^{2}\right)\right\}^{1 / 2} d t=\left\{\sum_{j=1}^{n}\left(\ln t_{j}\right)^{2}\right\}^{1 / 2} .
$$

We put

$$
\beta(t)=\left(\beta_{k j}(t)\right) \text { with } 0 \leq t \leq 1,1 \leq k \leq h, 1 \leq j \leq g .
$$

Then we obtain

$$
\begin{aligned}
& \int_{0}^{1}\left\{\sigma\left(\alpha(t)^{-1}\left(\frac{d \beta}{d t}\right) \frac{d \beta}{d t}\right)\right\}^{1 / 2} d t \\
= & \int_{0}^{1}\left\{\sigma\left(F^{-1}\left(\frac{d R}{d t}\right) \frac{d R}{d t}\right)+\sigma\left(G^{-1}\left(\frac{d S}{d t}\right) \frac{d S}{d t}\right)\right\}^{1 / 2} d t \\
= & \int_{0}^{1}\left\{\sum_{j=1}^{g} \sum_{k=1}^{h} e^{-t \ln t_{j}}\left(\frac{d \beta_{k j}}{d t}\right)^{2}\right\}^{1 / 2} d t .
\end{aligned}
$$


Each curve $\beta_{k j}(t)(0 \leq t \leq 1)$ is a curve in $\mathbb{R}$ such that $\beta_{k} j(0)=0$ and $\beta_{k j}(1)=\widetilde{v}_{k j}$. Thus each curve $\beta_{k j}(t)$ must be a straight line, that is, for all $k, j$ with $1 \leq k \leq h$ and $1 \leq j \leq g$,

$$
\beta_{k j}(t)=\widetilde{v}_{k j} t, \quad 0 \leq t \leq 1 .
$$

Therefore we have

$$
\begin{aligned}
& \int_{0}^{1}\left\{\sigma\left(\alpha(t)^{-1}\left(\frac{d \beta}{d t}\right) \frac{d \beta}{d t}\right)\right\}^{1 / 2} d t \\
= & \int_{0}^{1}\left\{\sum_{j=1}^{n} e^{-t \ln t_{j}}\left(\sum_{k=1}^{h} \widetilde{v}_{k j}^{2}\right)\right\}^{1 / 2} d t \\
= & \int_{0}^{1}\left(\sum_{j=1}^{g} \Delta_{j} e^{-t \ln t_{j}}\right)^{1 / 2} d t .
\end{aligned}
$$

Finally we obtain

$$
s\left(\left(Y_{0}, V_{0}\right),\left(Y_{1}, V_{1}\right)\right)=A\left\{\sum_{j=1}^{g}\left(\ln t_{j}\right)^{2}\right\}^{1 / 2}+B \int_{0}^{1}\left(\sum_{j=1}^{g} \Delta_{j} e^{-\left(\ln t_{j}\right) t}\right)^{1 / 2} d t
$$

Hence we complete the proof.

For a fixed element $(A, a) \in G L_{g, h}$, we let $\Theta_{A, a}: \mathcal{P}_{g} \times \mathbb{R}^{(h, g)} \longrightarrow \mathcal{P}_{g} \times \mathbb{R}^{(h, g)}$ be the mapping defined by

$$
\Theta_{A, a}(Y, V):=(A, a) \cdot(Y, V), \quad(Y, V) \in \mathcal{P}_{g} \times \mathbb{R}^{(h, g)} .
$$

We consider the behaviour of the differential map $d \Theta_{A, a}$ of $\Theta_{A, a}$ at $\left(I_{g}, 0\right)$. Then $d \Theta_{A, a}$ is given by

$$
d \Theta_{A, a}(u, v)=\left(A u^{t} A, v^{t} A\right),
$$

where $(u, v)$ is a tangent vector of $\mathcal{P}_{g} \times \mathbb{R}^{(h, g)}$ at $\left(I_{g}, 0\right)$.

We let $\tilde{\theta}$ be the involution of $G L_{g, h}$ defined by

$$
\tilde{\theta}((A, a)):=\left({ }^{t} A^{-1},-a\right), \quad(A, a) \in G L_{g, h} .
$$

Then the differential map of $\tilde{\theta}$ at $\left(I_{g}, 0\right)$, denoted by the same notation $\tilde{\theta}$ is given by

$$
\tilde{\theta}: \mathfrak{g} \longrightarrow \mathfrak{g}, \quad \tilde{\theta}(X, Z)=\left(-{ }^{t} X,-Z\right),
$$

where $X \in \mathbb{R}^{(g, g)}$ and $Z \in \mathbb{R}^{(h, g)}$. We note that $\mathfrak{k}$ is the $(+1)$-eigenspace of $\tilde{\theta}$ and

$$
\mathfrak{p}=\left\{(X, Z) \mid X \in \mathbb{R}^{(g, g)}, X={ }^{t} X, Z \in \mathbb{R}^{(h, g)}\right\}
$$

is the $(-1)$-eigenspace of $\tilde{\theta}$. 
Now we consider some differential forms on $\mathcal{P}_{g} \times \mathbb{R}^{(h, g)}$ which are invariant under the action of $G L(g, \mathbb{Z}) \ltimes \mathbb{Z}^{(h, g)}$. We let

$$
\mathfrak{G}_{g, h}:=G L(g, \mathbb{Z}) \ltimes \mathbb{Z}^{(h, g)}
$$

be the discrete subgroup of $G L_{g, h}$. Let

$$
\alpha_{*}=\sum_{\mu \leq \nu} f_{\mu \nu}(Y, V) d y_{\mu \nu}+\sum_{k=1}^{h} \sum_{l=1}^{g} \phi_{k l}(Y, V) d v_{k l}
$$

be a differential 1 -form on $\mathcal{P}_{g} \times \mathbb{R}^{(h, g)}$ that is invariant under the action of $\mathfrak{G}_{g, h}$. We put

$$
e_{\mu \nu}= \begin{cases}1 & \text { if } \mu=\nu \\ \frac{1}{2} & \text { otherwise. }\end{cases}
$$

We let

$$
f(Y, V)=\left(e_{\mu \nu} f_{\mu \nu}(Y, V)\right) \text { and } \phi(Y, V)={ }^{t}\left(\phi_{k l}(Y, V)\right),
$$

where $f(Y, V)$ is a $g \times g$ matrix with entries $f_{\mu \nu}(Y, V)$ and $\phi(Y, V)$ is a $g \times h$ matrix with entries $\phi_{k l}(Y, V)$. Then

$$
\alpha_{*}=\sigma(f d Y+\phi d V) .
$$

If $\tilde{\gamma}=(\gamma, \alpha) \in \mathfrak{G}_{g, h}$ with $\gamma \in G L(g, \mathbb{Z})$ and $\alpha \in \mathbb{Z}^{(h, g)}$, then we have the following transformation relation

$$
f\left(\gamma Y^{t} \gamma,(V+\alpha){ }^{t} \gamma\right)={ }^{t} \gamma^{-1} f(Y, V) \gamma^{-1}
$$

$$
\phi\left(\gamma Y^{t} \gamma,(V+\alpha){ }^{t} \gamma\right)={ }^{t} \gamma^{-1} \phi(Y, V)
$$

We let

$$
\omega_{0}=d y_{11} \wedge d y_{12} \wedge \cdots \wedge d y_{n n} \wedge d v_{11} \wedge \cdots \wedge d v_{h g}
$$

be a differential form on $\mathcal{P}_{g} \times \mathbb{R}^{(h, g)}$ of degree $\widetilde{N}:=\frac{g(g+1)}{2}+g h$. If $\omega=h(Y, V) \omega_{0}$ is a differential form on $\mathcal{P}_{g} \times \mathbb{R}^{(h, g)}$ of degree $\tilde{N}$ that is invariant under the action of $\mathfrak{G}_{g, h}$. Then the function $h(Y, V)$ satisfies the transformation relation

$$
h\left(\gamma Y^{t} \gamma,(V+\alpha)^{t} \gamma\right)=(\operatorname{det} \gamma)^{-(g+h+1)} h(Y, V)
$$

for all $\gamma \in G L(g, \mathbb{Z})$ and $\alpha \in \mathbb{Z}^{(h, g)}$.

We write

$$
\omega_{1}=d y_{11} \wedge d y_{12} \wedge \cdots \wedge d y_{g g} \quad \text { and } \quad \omega_{2}=d v_{11} \wedge \cdots \wedge d v_{h g} .
$$

Now we define

$$
\omega_{a b}=\epsilon_{a b} \bigwedge_{\substack{1 \leq \mu \leq \nu \leq g \\(\mu, \nu) \neq(a, b)}} d y_{\mu \nu} \wedge \omega_{2}, \quad 1 \leq a \leq b \leq g
$$

and

$$
\tilde{\omega}_{c d}=\tilde{\epsilon}_{c d} \omega_{1} \wedge \bigwedge_{\substack{1 \leq k \leq h, 1 \leq l \leq g \\(k, l) \neq(c, d)}} d v_{k l}, \quad 1 \leq c \leq h, 1 \leq d \leq g .
$$


Here the signs $\epsilon_{a b}$ and $\tilde{\epsilon}_{c d}$ are determined by the relations $\epsilon_{a b} \omega_{a b} \wedge d y_{a b}=\omega_{0}$ and $\tilde{\epsilon}_{c d} \omega_{c d} \wedge d v_{c d}=\omega_{0}$. We let

$$
\beta_{*}=\sum_{\mu \leq \nu} s_{\mu \nu}(Y, V) \omega_{\mu \nu}+\sum_{k=1}^{h} \sum_{l=1}^{g} \varphi_{k l}(Y, V) \tilde{\omega}_{k l}
$$

be a differential form on $\mathcal{P}_{g} \times \mathbb{R}^{(h, g)}$ of degree $\widetilde{N}-1$ that is invariant under the action of $\mathfrak{G}_{g, h}$, where $s_{\mu \nu}(Y, V)$ and $\varphi_{k l}$ are smooth functions on $\mathcal{P}_{g} \times \mathbb{R}^{(h, g)}$. We set

$$
s=\left(\epsilon_{\mu \nu} s_{\mu \nu}\right), \epsilon_{\mu \nu}=\epsilon_{\nu \mu}, s_{\mu \nu}=s_{\nu \mu} \quad \text { and } \quad \varphi=\left(\tilde{\epsilon}_{k l} \varphi_{k l}\right) .
$$

If we write

then we obtain

$$
\Omega(Y, V)=\left(\begin{array}{c}
s(Y, V) \\
\varphi(Y, V)
\end{array}\right)
$$

$$
\beta_{*} \wedge\left(\begin{array}{l}
d Y \\
d V
\end{array}\right)=\Omega \omega_{0} .
$$

If $\tilde{\gamma}=(\gamma, \alpha) \in \mathfrak{G}_{g, h}$, then we have the following transformation relations:

$$
s\left(\gamma Y^{t} \gamma,(V+\alpha)^{t} \gamma\right)=(\operatorname{det} \gamma)^{-(g+h+1)} \gamma s(Y, V)^{t} \gamma
$$

and

$$
\varphi\left(\gamma Y^{t} \gamma,(V+\alpha)^{t} \gamma\right)=(\operatorname{det} \gamma)^{-(g+h+1)} \varphi(Y, V)^{t} \gamma
$$

$\mathfrak{G}_{g, h}$ acts on $\mathcal{P}_{g} \times \mathbb{R}^{(h, g)}$ properly discontinuously. The quotient space

$$
\mathfrak{G}_{g, h} \backslash\left(\mathcal{P}_{g} \times \mathbb{R}^{(h, g)}\right)
$$

may be regarded as a family of principally polarized real tori of dimension $g h$. To each equivalence class $[Y] \in \mathfrak{G}_{g} \backslash \mathcal{P}_{g}$ with $Y \in \mathcal{P}_{g}$ we associate a principally polarized real torus $T_{Y}^{[h]}=T_{Y} \times \cdots \times T_{Y}$ with $T=\mathbb{R}^{g} / \Lambda_{Y}$, where $\Lambda_{Y}=Y \mathbb{Z}^{g}$ is a lattice in $\mathbb{R}^{g}$.

Let $Y_{1}$ and $Y_{2}$ be two elements in $\mathcal{P}_{g}$ with $\left[Y_{1}\right] \neq\left[Y_{2}\right]$, that is, $Y_{2} \neq A Y_{1}{ }^{t} A$ for all $A \in \mathfrak{G}_{g}$. We put $\Lambda_{i}=Y_{i} \mathbb{Z}^{g}$ for $i=1,2$. Then a torus $T_{1}=\mathbb{R}^{g} / \Lambda_{1}$ is diffeomorphic to $T_{2}=\mathbb{R}^{g} / \Lambda_{2}$ as smooth manifolds but $T_{1}$ is not isomorphic to $T_{2}$ as polarized tori.

Lemma 8.4. The following set

$$
\mathfrak{R}_{g, h}:=\left\{(Y, V)\left|Y \in \mathfrak{R}_{g},\right| v_{k j} \mid \leq 1, V=\left(v_{k j}\right) \in \mathbb{R}^{(h, g)}\right\}
$$

is a fundamental set for $\mathfrak{G}_{g, h} \backslash \mathcal{P}_{g} \times \mathbb{R}^{(h, g)}$.

Proof. It is easy to see that $\mathfrak{R}_{g, h}$ is a fundamental set for $\mathfrak{G}_{g, h} \backslash \mathcal{P}_{g} \times \mathbb{R}^{(h, g)}$. We leave the detail to the reader. 
For two positive integers $g$ and $h$, we consider the Heisenberg group

$$
H_{\mathbb{R}}^{(g, h)}=\left\{(\lambda, \mu ; \kappa) \mid \lambda, \mu \in \mathbb{R}^{(h, g)}, \kappa \in \mathbb{R}^{(h, h)}, \kappa+\mu^{t} \lambda \text { symmetric }\right\}
$$

endowed with the following multiplication law

$$
(\lambda, \mu ; \kappa) \circ\left(\lambda^{\prime}, \mu^{\prime} ; \kappa^{\prime}\right)=\left(\lambda+\lambda^{\prime}, \mu+\mu^{\prime} ; \kappa+\kappa^{\prime}+\lambda^{t} \mu^{\prime}-\mu^{t} \lambda^{\prime}\right) .
$$

We define the semidirect product of $S p(g, \mathbb{R})$ and $H_{\mathbb{R}}^{(g, h)}$

$$
G^{J}=S p(g, \mathbb{R}) \ltimes H_{\mathbb{R}}^{(g, h)}
$$

endowed with the following multiplication law

$(M,(\lambda, \mu ; \kappa)) \cdot\left(M^{\prime},\left(\lambda^{\prime}, \mu^{\prime} ; \kappa^{\prime}\right)\right)=\left(M M^{\prime},\left(\tilde{\lambda}+\lambda^{\prime}, \tilde{\mu}+\mu^{\prime} ; \kappa+\kappa^{\prime}+\tilde{\lambda}^{t} \mu^{\prime}-\tilde{\mu}^{t} \lambda^{\prime}\right)\right)$

with $M, M^{\prime} \in S p(g, \mathbb{R}),(\lambda, \mu ; \kappa),\left(\lambda^{\prime}, \mu^{\prime} ; \kappa^{\prime}\right) \in H_{\mathbb{R}}^{(g, h)}$ and $(\tilde{\lambda}, \tilde{\mu})=(\lambda, \mu) M^{\prime}$. Then $G^{J}$ acts on the Siegel-Jacobi space $\mathbb{H}_{g} \times \mathbb{C}^{(h, g)}$ transitively by

$$
(M,(\lambda, \mu ; \kappa)) \cdot(\Omega, Z)=\left(M \cdot \Omega,(Z+\lambda \Omega+\mu)(C \Omega+D)^{-1}\right),
$$

where $M=(\underset{C}{A} \stackrel{B}{D}) \in S p(g, \mathbb{R}),(\lambda, \mu ; \kappa) \in H_{\mathbb{R}}^{(g, h)}$ and $(\Omega, Z) \in \mathbb{H}_{g} \times \mathbb{C}^{(h, g)}$. We note that the Jacobi group $G^{J}$ is not a reductive Lie group and also that the space $\mathbb{H}_{g} \times \mathbb{C}^{(h, g)}$ is not a symmetric space. We refer to $[29,30,31,32,33,34]$ for more detail on the Siegel-Jacobi space $\mathbb{H}_{g} \times \mathbb{C}^{(h, g)}$.

We let

$$
\Gamma_{g, h}:=\Gamma_{g} \ltimes H_{\mathbb{Z}}^{(g, h)}
$$

be the discrete subgroup of $G^{J}$, where

$$
H_{\mathbb{Z}}^{(g, h)}=\left\{(\lambda, \mu ; \kappa) \in H_{\mathbb{R}}^{(g, h)} \mid \lambda, \mu \in \mathbb{Z}^{(h, g)}, \quad \kappa \in \mathbb{Z}^{(h, h)}\right\} .
$$

We define the map $\Phi_{g, h}: \mathcal{P}_{g} \times \mathbb{R}^{(h, g)} \longrightarrow \mathbb{H}_{g} \times \mathbb{C}^{(h, g)}$ by

$$
\Phi_{g, h}(Y, \zeta):=(i Y, \zeta), \quad(Y, \zeta) \in \mathcal{P}_{g} \times \mathbb{R}^{(h, g)} .
$$

We have the following inclusions

$$
\mathcal{P}_{g} \times \mathbb{R}^{(h, g)} \stackrel{\Phi_{g, h}}{\longrightarrow} \mathscr{H}_{g} \times \mathbb{C}^{(h, g)} \hookrightarrow \mathbb{H}_{g} \times \mathbb{C}^{(h, g)} \hookrightarrow \mathbb{H}_{g}^{*} \times \mathbb{C}^{(h, g)} .
$$

$\mathfrak{G}_{g, h}$ acts on $\mathcal{P}_{g} \times \mathbb{R}^{(h, g)}, \Gamma_{g}^{\star} \ltimes H_{\mathbb{Z}}^{(g, h)}$ acts on $\mathscr{H}_{g} \times \mathbb{C}^{(h, g)}$ and $\Gamma_{g, h}$ acts on $\mathbb{H}_{g} \times \mathbb{C}^{(h, g)}$ and $\mathbb{H}_{g}^{*} \times \mathbb{C}^{(h, g)}$. It might be interesting to characterize the boundary points of the closure of the image of $\Phi_{g, h}$ in $\mathbb{H}_{g}^{*} \times \mathbb{C}^{(h, g)}$.

\section{Real semi-abelian varieties}

In this section we review the work of Silhol on semi-abelian varieties [26] which is needed in the next section.

Definition. A complex semi-abelianvariety $A$ is the extension of an abelian variety $\widetilde{A}$ by a group of multiplicative type. A semi-abelian variety is said to be real if it admits an anti-holomorphic involution which is a group homomorphism. 
Let $T$ be a group of multiplicative type. We consider the exponential map $\exp : \mathfrak{t} \longrightarrow T$. The real structure $S$ on $T$ lifts to a real structure $S_{\mathfrak{t}}$ on $\mathfrak{t}$. Then $L_{\mathfrak{t}}:=$ ker exp is a free $\mathbb{Z}$-module and $S_{\mathfrak{t}}$ induces an involution on $L_{\mathfrak{t}}$. By standard results (cf. [25, I. (3.5.1)]), we can find a basis of $L_{\mathfrak{t}}$ with respect to which the matrix for $S_{\mathfrak{t}}$ is of the form

$$
\left(\begin{array}{cccccc}
I_{s} & 0 & 0 & \cdots & 0 & 0 \\
0 & B & 0 & \cdots & 0 & 0 \\
0 & 0 & B & \cdots & 0 & 0 \\
0 & 0 & 0 & \ddots & 0 & 0 \\
0 & 0 & 0 & \cdots & B & 0 \\
0 & 0 & 0 & \cdots & 0 & -I_{t}
\end{array}\right), \quad B:=\left(\begin{array}{cc}
0 & 1 \\
1 & 0
\end{array}\right) .
$$

Since fixing a basis of $L_{\mathfrak{t}}$ is equivalent to fixing an isomorphism $T \cong\left(\mathbb{C}^{*}\right)^{r}$, we get

$$
T=T_{1} \times T_{2} \times T_{3}, \quad r=s^{\prime}+2 p+t^{\prime},
$$

where

(i) $T_{1}=\mathbb{C}^{*} \times \cdots \times \mathbb{C}^{*}\left(s^{\prime}\right.$-times $)$ and $S$ induces on each factor the involution $z \longmapsto \bar{z}$. In this case we write $T_{1}=\mathbb{G}_{m}^{0} \times \cdots \times \mathbb{G}_{m}^{0}$;

(ii) $T_{2}=\mathbb{C}^{*} \times \cdots \times \mathbb{C}^{*}\left(t^{\prime}\right.$-times) and $S$ induces on each factor the involution $z \longmapsto \bar{z}^{-1}$. In this case we write $T_{1}=\mathbb{G}_{m}^{\infty} \times \cdots \times \mathbb{G}_{m}^{\infty}$;

(iii) $T_{3}=\left(\mathbb{C}^{*} \times \mathbb{C}^{*}\right) \times \cdots \times\left(\mathbb{C}^{*} \times \mathbb{C}^{*}\right)(p$-times $)$ and $S$ induces on each factor $\left(\mathbb{C}^{*} \times \mathbb{C}^{*}\right)$ the involution $\left(z_{1}, z_{2}\right) \longmapsto\left(\bar{z}_{2}, \bar{z}_{1}\right)$. In this case we write $T_{3}=\mathbb{G}_{m}^{2 *} \times \cdots \times \mathbb{G}_{m}^{2 *}$.

Let $\Delta=\{\zeta \in \mathbb{C}|| \zeta \mid<1\}$ be the unit disk and let $\Delta^{*}=\{\zeta \in \mathbb{C}|0<| \zeta \mid<1\}$ be a punctured unit disk. Let $\varphi: Z^{*} \longrightarrow \Delta^{*}$ be a holomorphic family of matrices $\varphi^{-1}(\zeta)=Z(\zeta)$ in $\mathbb{H}_{g}$. We have the natural action of the lattice $\mathbb{Z}^{2 g}$ on $\Delta^{*} \times \mathbb{C}^{g}$ defined by

$$
(\lambda, \mu) \cdot(\zeta, z):=(\zeta, z+\lambda+Z(\zeta) \mu), \quad \zeta \in \Delta^{*}, \lambda, \mu \in \mathbb{Z}^{g}, z \in \mathbb{C}^{g} .
$$

Then the quotient space

$$
\mathbf{A}^{*}:=\left(\Delta^{*} \times \mathbb{C}^{g}\right) / \mathbb{Z}^{2 g}
$$

is a holomorphic family of principally polarized abelian varieties associated to a holomorphic family $\varphi: Z^{*} \longrightarrow \Delta^{*}$.

Now we write

$$
Z(\zeta)=X(\zeta)+i Y(\zeta) \in \mathbb{H}_{g}
$$

and

$$
Y(\zeta)={ }^{t} W(\zeta) D(\zeta) W(\zeta) \in \mathcal{P}_{g} \quad \text { (the Jacobi decomposition) }
$$

with $\operatorname{diag}\left(d_{1}(\zeta), \ldots, d_{g}(\zeta)\right) \in \mathbb{R}^{(g, g)}$ is a diagonal matrix.

Now we assume the following conditions (F1)-(F3): for any $\zeta \in \Delta_{r}^{*}:=\{\zeta \in$ $\mathbb{C}|0<| \zeta \mid<r\}$

(F1) There exists a positive number $r>0$ such that for any $\zeta \in \Delta_{r}^{*}, Z(\zeta) \in$ $\mathfrak{W}_{g}(u)$ for some $u>0$, where $\Delta_{r}^{*}:=\{\zeta \in \mathbb{C}|0<| \zeta \mid<r\}$; 
(F2) $X(\zeta)$ converges in $\mathbb{R}^{(g, g)}$ and $W(\zeta)$ converges in $G L(g, \mathbb{R})$ as $\zeta \rightarrow 0$;

(F3) $\lim _{\zeta \rightarrow 0} d_{i}(\zeta)=d_{i}$ converges for $1 \leq i \leq g-t$, and $\lim _{\zeta \rightarrow 0} d_{i}(\zeta)=\infty$ for $g-t<i \leq g$.

Let

$$
Z(0)=\left(\begin{array}{cccccc}
z_{11} & \cdots & z_{1, g-t} & 0 & \cdots & 0 \\
\vdots & \ddots & \vdots & 0 & \ddots & \vdots \\
z_{g-t, 1} & \cdots & z_{g-t, g-t} & 0 & \cdots & 0 \\
\vdots & \ddots & \vdots & \vdots & \cdots & \vdots \\
z_{g, 1} & \cdots & z_{g, g-t} & 0 & \cdots & 0
\end{array}\right), \quad z_{i j}=\lim _{\zeta \rightarrow 0} z_{i j}(\zeta) .
$$

The action (9.1) extends to the action of $\mathbb{Z}^{2 g}$ on $\Delta \times \mathbb{C}^{g}$ by letting $Z(0)$ be the fibre at $\zeta=0$. We take the quotient space

$$
\mathbf{A}:=\left(\Delta \times \mathbb{C}^{g}\right) / \mathbb{Z}^{2 g} .
$$

Then we see that $\mathbf{A}$ is an analytic variety fibred holomorphically over $\Delta$, and the fibre at 0 is a semi-abelian variety

$$
\mathbf{A}_{0}=\mathbb{C}^{g} / L_{0}, \quad L_{0}:=\mathbb{Z}^{g} Z(0)+\mathbb{Z}^{g} \subset \mathbb{C}^{g}
$$

of the abelian variety

$$
\widetilde{\mathbf{A}}_{0}:=\mathbb{C}^{g-t} / L^{\diamond}, \quad L^{\diamond}:=\mathbb{Z}^{g-t} Z^{\diamond}(0)+\mathbb{Z}^{g-t} \subset \mathbb{C}^{g-t}
$$

by $\left(\mathbb{C}^{*}\right)^{t}$, where

$$
Z^{\diamond}(0)=\left(\begin{array}{ccc}
z_{11} & \cdots & z_{1, g-t} \\
\vdots & \ddots & \vdots \\
z_{g-t, 1} & \cdots & z_{g-t, g-t}
\end{array}\right) \in \mathbb{H}_{g-t} .
$$

The extension

$$
1 \longrightarrow\left(\mathbb{C}^{*}\right)^{t} \longrightarrow \mathbf{A}_{0} \longrightarrow \widetilde{\mathbf{A}}_{0} \longrightarrow 0
$$

is defined by the image of

$$
z_{g-k}^{\diamond}=\left(z_{g-k, 1}, \ldots, z_{g-k, g-t}\right) \in \mathbb{C}^{g-t}, \quad k=0, \ldots, t-1
$$

under the maps

$$
\mathbb{C}^{g-t} \longrightarrow \widetilde{\mathbf{A}}_{0} \longrightarrow \operatorname{Pic}^{0}\left(\widetilde{\mathbf{A}}_{0}\right),
$$

where the last map is the isomorphism defined by the polarization.

These above facts can be generalized as follows.

Proposition 9.1. Let $\varphi: Z^{*} \longrightarrow \Delta^{*}$ be a holomorphic family of matrices $\varphi^{-1}(\zeta)=Z(\zeta)$ in $\mathbb{H}_{g}$ such that $\varphi^{-1}(\zeta)=Z(\zeta)$ converges in $\mathbb{H}_{g}^{*}$ as $\zeta \rightarrow 0$. Then there exists an analytic variety $\boldsymbol{A}\left(Z^{*}\right) \longrightarrow \Delta$ such that

(i) the fibre at $\zeta(\neq 0) \in \Delta$ is the principally polarized abelian variety $\mathbb{C}^{g} / L_{\zeta}$ with the lattice $L_{\zeta}=\mathbb{Z}^{g} Z(\zeta)+\mathbb{Z}^{g}$;

(ii) the zero fibre $\boldsymbol{A}\left(Z^{*}\right)_{0}$ is a semi-abelian variety.

Proof. The proof can be found in [26, p. 189]. 
Theorem 9.1. Let $\varphi: Z^{*} \longrightarrow \Delta^{*}$ be a holomorphic family of matrices $\varphi^{-1}(\zeta)=Z(\zeta)$ in $\mathbb{H}_{g}$ such that $\varphi^{-1}(\zeta)=Z(\zeta)$ converges in $\mathbb{H}_{g}^{*}$ as $\zeta \rightarrow 0$. We assume that $Z(\zeta)=\varphi^{-1}(\zeta) \in \mathscr{H}_{g}$ for $\zeta \in \mathbb{R} \cap \Delta^{*}$. Let $(s, t)$ be such that

$$
\lim _{\zeta \rightarrow 0} Z(\zeta) \in \gamma B_{M}\left(\mathscr{F}_{s, t} \cap \overline{\mathscr{H}_{0}}\right)
$$

for some $M \in \mathbb{Z}^{(g, g)}$ and some $\gamma \in \Gamma_{g}^{\star}$. Then

(a) $\boldsymbol{A}\left(Z^{*}\right)_{0}$ has a natural real structure extending the real structures of the $\boldsymbol{A}\left(Z^{*}\right)_{\zeta}{ }^{\prime} s$ for $\zeta \in \mathbb{R} \cap \Delta^{*}$

(b) As a real variety, $\boldsymbol{A}\left(Z^{*}\right)_{0}$ is the extension of a real abelian variety $\widetilde{\boldsymbol{A}}\left(Z^{*}\right)_{0}$ by

$$
\left(\mathbb{G}_{m}^{0}\right)^{s^{\prime}} \times\left(\mathbb{G}_{m}^{2 *}\right)^{p} \times\left(\mathbb{G}_{m}^{\infty}\right)^{t^{\prime}}, \quad s=s^{\prime}+p, t=t^{\prime}+p ;
$$

(c) Let $x \in \mathscr{X}_{\mathbb{R}}^{g}(s, t) \subset \overline{\mathscr{X}_{\mathbb{R}}^{g}}$ be the image of $\lim _{\zeta \rightarrow 0} Z(\zeta)$ in $\overline{\mathscr{X}_{\mathbb{R}}^{g}}$ and let $[x]$ be the image of $x$ under the isomorphism $\mathscr{X}_{\mathbb{R}}^{g}(s, t) \cong \mathscr{X}_{\mathbb{R}}^{g-r}$ with $r=s+t$. Then $[x]$ is the real isomorphism class of $\widetilde{\boldsymbol{A}}\left(Z^{*}\right)_{0}$.

Proof. The proof can be found in [26, pp. 191-192].

Corollary 9.1. Let $\varphi: Z^{*} \longrightarrow \Delta^{*}$ be as in Theorem 9.1. Assume

$$
\lim _{\zeta \longrightarrow 0} Z(\zeta) \in \mathscr{F}_{0, t} \quad\left(\text { resp. } \mathscr{F}_{s, 0}\right) \text {. }
$$

Then the class of the extension

$$
\begin{gathered}
0 \longrightarrow\left(\mathbb{G}_{m}^{\infty}\right)^{t} \rightarrow \boldsymbol{A}\left(Z^{*}\right)_{0} \longrightarrow \widetilde{\boldsymbol{A}}\left(Z^{*}\right)_{0} \longrightarrow 0 \\
\left(\text { resp. } 0 \longrightarrow\left(\mathbb{G}_{m}^{0}\right)^{s} \longrightarrow \boldsymbol{A}\left(Z^{*}\right)_{0} \longrightarrow \widetilde{\boldsymbol{A}}\left(Z^{*}\right)_{0} \longrightarrow 0\right)
\end{gathered}
$$

is defined by $t$ purely imaginary divisors on $\boldsymbol{A}\left(Z^{*}\right)_{0}$ (resp. $s$ real divisors $\left.\boldsymbol{A}\left(Z^{*}\right)_{0}\right)$.

\section{Real semi-tori}

A real semi-torus $T$ of dimension $g$ is defined to be an extension of a real torus $\widetilde{T}$ of dimension $g-t$ by a real group $\left(\mathbb{R}^{*}\right)^{t}$ of multiplicative type, where $\mathbb{R}^{*}=\mathbb{R}-\{0\}$.

Let $I=\{\xi \in \mathbb{R} \mid-1<\xi<1\}$ be the unit interval and $I^{*}=I-\{0\}$ be the punctured unit interval. Let $\varpi: \mathfrak{Y}^{*} \longrightarrow I^{*}$ be a real analytic family of matrices $\varpi^{-1}(\xi)=Y(\xi) \in \mathcal{P}_{g}$. We have the natural action of the lattice $\mathbb{Z}^{g}$ in $\mathbb{R}^{g}$ on $I^{*} \times \mathbb{R}^{g}$ defined by

$$
\alpha \cdot(\xi, x)=(\xi, x+Y(\xi) \alpha), \quad \alpha \in \mathbb{Z}^{g}, \xi \in I^{*}, x \in \mathbb{R}^{g} .
$$

The quotient space

$$
\mathbf{T}^{*}:=\left(I^{*} \times \mathbb{R}^{g}\right) / \mathbb{Z}^{g}
$$


is a real analytic family of real tori of dimension $g$ associated to a real analytic family $\varpi: \mathfrak{Y}^{*} \longrightarrow I^{*}$. We let

$$
Y(\xi)={ }^{t} W(\xi) D(\xi) W(\xi)
$$

be the Jacobi decomposition of $Y(\xi)$, where $D(\xi)=\operatorname{diag}\left(d_{1}(\xi), \ldots, d_{g}(\xi)\right)$ is a real diagonal matrix and $W(\xi)$ is a strictly upper triangular real matrix of degree $g$. Now we assume the following conditions (T1)-(T4):

(T1) There exists a positive number $r$ with $0<r<1$ such that for any $\xi \in I_{r}^{*}, i Y(\xi) \in \mathfrak{W}_{g}(u)$ for some $u>0$, where $I_{r}^{*}:=\{\xi \in \mathbb{R} \mid-r<\xi<r\}$;

(T2) $W(\xi)$ converges in $G L(g, \mathbb{R})$ as $\xi \rightarrow 0$;

(T3) $\lim _{\xi \rightarrow 0} d_{i}(\xi)=d_{i}$ converges for $1 \leq i \leq g-t$, and $\lim _{\xi \rightarrow 0} d_{i}(\xi)=\infty$ for $g-t<i \leq g$.

Let

$$
Y(0)=\left(\begin{array}{cccccc}
y_{11} & \cdots & y_{1, g-t} & 0 & \cdots & 0 \\
\vdots & \ddots & \vdots & 0 & \ddots & \vdots \\
y_{g-t, 1} & \cdots & y_{g-t, g-t} & 0 & \cdots & 0 \\
\vdots & \ddots & \vdots & \vdots & \cdots & \vdots \\
y_{g, 1} & \cdots & y_{g, g-t} & 0 & \cdots & 0
\end{array}\right), \quad y_{i j}=\lim _{\xi \rightarrow 0} y_{i j}(\zeta)
$$

The action (10.1) extends to the action of $\mathbb{Z}^{g}$ on $I \times \mathbb{R}^{g}$ by letting $Y(0)$ be the fibre at $\xi=0$. We take the quotient space

$$
\mathbf{T}:=\left(I \times \mathbb{R}^{g}\right) / \mathbb{Z}^{g} .
$$

Then we see that $\mathbf{T}$ is a real analytic variety fibred real analytically over $I$, and the fibre at 0 is a real semi-torus

$$
\mathbf{T}_{0}=\mathbb{R}^{g} / \Lambda_{0}, \quad \Lambda_{0}:=\mathbb{Z}^{g} Y(0) \subset \mathbb{R}^{g}
$$

of the real torus

$$
\widetilde{\mathbf{T}}_{0}:=\mathbb{R}^{g-t} / \Lambda^{\diamond}, \quad \Lambda^{\diamond}:=\mathbb{Z}^{g-t} Y^{\diamond}(0) \text { is a lattice in } \mathbb{R}^{g-t}
$$

by $\left(\mathbb{C}^{*}\right)^{t}$, where

$$
Y^{\diamond}(0)=\left(\begin{array}{ccc}
y_{11} & \cdots & y_{1, g-t} \\
\vdots & \ddots & \vdots \\
y_{g-t, 1} & \cdots & y_{g-t, g-t}
\end{array}\right) \in \mathcal{P}_{g-t} .
$$

\section{Open problems and remarks}

In this final section we give some open problems related to polarized real tori to be studied in the future.

Problem 1. Characterize the boundary points of the closure of $i \mathcal{P}_{g}$ in $\mathbb{H}_{g}^{*}$ explicitly.

Problem 2. Find the explicit generators of the ring $\mathbb{D}(g, h)$ of differential operators on the Minkowski-Euclidean space $\mathcal{P}_{g} \times \mathbb{R}^{(h, g)}$ which are invariant under the action $(8.7)$ of $G L_{g, h}=G L(g, \mathbb{R}) \ltimes \mathbb{R}^{(h, g)}$. 
Problem 3. Find all the relations among a complete explicit list of generators of $\mathbb{D}(g, h)$.

The orthogonal group $O(g)$ of degree $g$ acts on the subspace

$$
\mathfrak{p}=\left\{(X, Z) \mid X={ }^{t} X \in \mathbb{R}^{(g, g)}, Z \in \mathbb{R}^{(h, g)}\right\}
$$

of the vector space $\mathbb{R}^{(g, g)} \times \mathbb{R}^{(h, g)}$ by

$$
k \cdot(X, Z)=\left(k X^{t} k, Z^{t} k\right), \quad k \in O(g),(X, V) \in \mathfrak{p} .
$$

The action (11.1) induces the action of $O(g)$ on the polynomial ring $\operatorname{Pol}(\mathfrak{p})$ on $\mathfrak{p}$. We denote by $I(\mathfrak{p})$ the subring of $\operatorname{Pol}(\mathfrak{p})$ consisting of polynomials on $\mathfrak{p}$ invariant under the action of $O(g)$. We see that there is a canonical linear bijection

$$
\Theta: I(\mathfrak{p}) \longrightarrow \mathbb{D}(g, h)
$$

of $I(\mathfrak{p})$ onto $\mathbb{D}(g, h)$. We refer to [9] and [35] for more detail.

Remark 11.1. M. Itoh [11] proved that $I(\mathfrak{p})$ is generated by $\alpha_{j}(1 \leq j \leq g)$ and $\beta_{p q}^{(k)}(0 \leq k \leq g-1,1 \leq p \leq q \leq h)$, where

$$
\alpha_{j}(X, Z)=\operatorname{tr}\left(X^{j}\right), \quad 1 \leq j \leq g
$$

and

$$
\beta_{p q}^{(k)}(X, Z)=\left(Z X^{k t} Z\right)_{p q}, \quad 0 \leq k \leq g-1,1 \leq p \leq q \leq h .
$$

Here $A_{p q}$ denotes the $(p, q)$-entry of a matrix $A$ of degree $h$.

Remark 11.2. M. Itoh [11] found all the relations among the above generators $\alpha_{j}(1 \leq j \leq g)$ and $\beta_{p q}^{(k)}(0 \leq k \leq g-1,1 \leq p \leq q \leq h)$ of $I(\mathfrak{p})$.

Problem 4. Develop the theory of harmonic analysis on the MinkowskiEuclidean space $\mathcal{P}_{g} \times \mathbb{R}^{(h, g)}$ with respect to a discrete subgroup of $G L(g, \mathbb{Z}) \ltimes$ $\mathbb{Z}^{(h, g)}$

Problem 5. Characterize the boundary points of the closure of the image of $\Phi_{g, h}$ in $\mathbb{H}_{g}^{*} \times \mathbb{C}^{(h, g)}$ (cf. see $(8.29)$ ).

Problem 6. Find the explicit generators of the ring $\mathbb{D}\left(\mathbb{H}_{g} \times \mathbb{C}^{(h, g)}\right)$ of differential operators on the Siegel-Jacobi space $\mathbb{H}_{g} \times \mathbb{C}^{(h, g)}$ which are invariant under the action (8.28) of the Jacobi group $G^{J}=S p(g, \mathbb{R}) \ltimes H_{\mathbb{R}}^{(g, h)}$. We refer to [34] for more detail.

Problem 7. Find all the relations among a complete list of generators of $\mathbb{D}\left(\mathbb{H}_{g} \times \mathbb{C}^{(h, g)}\right)$.

Problem 8. Develop the theory of harmonic analysis on the Siegel-Jacobi space $\mathbb{H}_{g} \times \mathbb{C}^{(h, g)}$ with respect to a congruent subgroup of $\Gamma_{g, h}=S p(g, \mathbb{Z}) \ltimes$ $H_{\mathbb{Z}}^{(g, h)}$. We refer to [32] for more detail. 


\section{Appendix: Non-abelian cohomology}

In this section we review some results on the first cohomology set $H^{1}(\langle\tau\rangle, \Gamma)$ obtained by Goresky and Tai [8], where $\langle\tau\rangle=\{1, \tau\}$ is a group of order 2 and $\gamma$ is a certain arithmetic subgroup. These results are often used in this article.

First of all we recall the basic definitions. Let $S$ be a group. A group $M$ is called a $S$-group if there exists an action of $G$ on $M, S \times M \longrightarrow M,(\sigma, a) \longmapsto$ $\sigma(a)$ such that $\sigma(a b)=\sigma(a) \sigma(b)$ for all $\sigma \in S$ and $a, b \in M$. From now on we let $1_{S}\left(\right.$ resp. $\left.1_{M}\right)$ be the identity element of $S$ (resp. $M$ ). We observe that if $M$ is a $S$-group, then $\sigma\left(1_{M}\right)=1_{M}$ for all $\sigma \in S$.

Definition. Let $M$ be a $S$-group, where $S$ is a group. We define

$$
H^{0}(S, M):=\{a \in M \mid \sigma(a)=a \text { for all } \sigma \in S\} .
$$

A map $f: S \longrightarrow M$ is called a 1-cocycle with values in $M$ if $f(\sigma \tau)=$ $f(\sigma) \sigma(f(\tau))$ for all $\sigma, \tau \in S$. We observe that if $f$ is a 1-cocycle, then $f\left(1_{S}\right)=$ $1_{M}$. We denote by $Z^{1}(S, M)$ the set of all 1-cocycles of $S$ with values in $M$. Let $f_{1}$ and $f_{2}$ be two 1-cocycles in $Z^{1}(S, M)$. We say that $f_{1}$ is cohomologous to $f_{2}$, denoted $f_{1} \sim f_{2}$, if there exists an element $h \in M$ such that

$$
f_{2}(\sigma)=h^{-1} f_{1}(\sigma) \sigma(h) \text { for all } \sigma \in S .
$$

Let $f_{\mathrm{b}}: S \longrightarrow M$ be the trivial map, i.e., $f_{\mathrm{b}}(\sigma)=1_{M}$ for all $\sigma \in S$. A map $f: S \longrightarrow M$ is called a 1-coboundary if $f \sim f_{b}$, i.e., if there exists $h \in M$ such that $f(\sigma)=h^{-1} \sigma(h)$ for all $\sigma \in S$.

Obviously a 1-coboundary is a 1-cocycle. It is easy to see that $\sim$ is an equivalence relation on $Z^{1}(S, M)$. So we define the first cohomology set

$$
H^{1}(S, M):=Z^{1}(S, M) / \sim .
$$

Remark. In general, $H^{1}(S, M)$ does not admit a group structure. But $H^{1}(S, M)$ has an identity, that is, the cohomologous class containing the trivial 1-cocycle $f_{b}$.

Example. Let $L$ be a Galois extension of a number field $K$ with Galois group $G$. A linear algebraic group defined over $K$ has naturally the structure of $G$ group. It is known that $H^{1}(G, G L(n, L))$ is trivial for all $n \geq 1$. Using the following exact sequence of $G$-groups

$$
1 \longrightarrow S L(n, L) \longrightarrow G L(n, L) \longrightarrow L^{*} \longrightarrow 1, \quad L^{*}=L-\{0\},
$$

we can show that $H^{1}(G, S L(n, L))$ is trivial.

We put $G=S p(g, \mathbb{R})$ and $K=U(g)$. Then $\mathbf{D}=G / K$ is biholomorphic to $\mathbb{H}_{g}$. Let $S_{\tau}=\{1, \tau\}$ be a group of order 2 as before. We define the $S_{\tau}$-group structure on $G$ via the action (2.7) of $S_{\tau}$ on $G$. Let $\Gamma$ be an arithmetic subgroup of $S p(g, \mathbb{Q})$. We let

$$
X_{\Gamma}:=\Gamma \backslash G / K \cong \Gamma \backslash \mathbb{H}_{g}
$$


and let $\pi_{\Gamma}: \mathbf{D} \longrightarrow X_{\Gamma}$ be the natural projection. For any $\gamma \in \Gamma$, we define the $\operatorname{map} f: S_{\tau} \longrightarrow \Gamma$ by

$$
f_{\gamma}(1)=1_{\Gamma} \quad \text { and } \quad f_{\gamma}(\tau)=\gamma,
$$

where $1_{\Gamma}$ denotes the identity element of $\Gamma$.

Lemma 1. Let $\gamma \in \Gamma$. Then

(a) $f_{\gamma}$ is a 1-cocycle if and only if $\gamma \tau(\gamma)=1_{\Gamma}$, equivalently, $\tau(\gamma) \gamma=1_{\Gamma}$.

(b) A cocycle $f_{\gamma}$ is a 1-coboundary if and only if there exists $h \in \Gamma$ such that $\gamma=\tau(h) h^{-1}$.

Proof. The proof follows immediately form the definition.

To each such a 1-cocycle $f_{\gamma}$ we associate the $\gamma$-twisted involution $\tau \gamma: \mathbf{D} \longrightarrow$ $\mathbf{D}$ and $\tau \gamma: \Gamma \longrightarrow \Gamma$. Indeed the involution $\tau \gamma: \mathbf{D} \longrightarrow \mathbf{D}$ is defined by

$$
\tau \gamma(x K)=\tau(\gamma x K)=\tau(\gamma) \tau(x) K, \quad x \in G
$$

and the involution $\tau \gamma: \Gamma \longrightarrow \Gamma$ is defined by

$$
\tau \gamma\left(\gamma_{1}\right)=\tau\left(\gamma \gamma_{1} \gamma^{-1}\right), \quad \gamma_{1} \in \Gamma .
$$

Let

$$
\mathbf{D}^{\tau \gamma}:=\{x \in \mathbf{D} \mid(\tau \gamma)(x)=x\}
$$

and

$$
\Gamma^{\tau \gamma}:=\left\{\gamma_{1} \in \Gamma \mid(\tau \gamma)\left(\gamma_{1}\right)=\gamma_{1}\right\}
$$

be the fixed point sets.

Lemma 2. Let $x \in \mathbf{D}$. Then $\pi_{\Gamma}(x) \in X_{\Gamma}^{\tau}$ if and only if there exists an element $\gamma \in \Gamma$ such that $x \in \mathbf{D}^{\tau \gamma}$.

Proof. It is easy to prove this lemma. We leave the proof to the reader.

Theorem A. Assume $\Gamma$ is torsion free. Let $\mathscr{C}_{\Gamma}$ be the set of all connected components of the fixed point set $X_{\Gamma}^{\tau}$. Then the map $\Phi_{\Gamma}: H^{1}\left(S_{\tau}, \Gamma\right) \longrightarrow \mathscr{C}_{\Gamma}$ defined by

$$
\Phi_{\Gamma}\left(\left[f_{\gamma}\right]\right):=\pi_{\Gamma}\left(\mathbf{D}^{\tau \gamma}\right)=\Gamma^{\tau \gamma} \backslash \mathbf{D}^{\tau \gamma}
$$

determines a one-to-one correspondence between $H^{1}\left(S_{\tau}, \Gamma\right)$ and $\mathscr{C}_{\Gamma}$.

Proof. The proof can be found in [8, pp. 3-4].

Theorem B. Let $S_{\tau}=\{1, \tau\}$ be a group of order 2 . Then $\operatorname{Sp}(g, \mathbb{R})$ has a $S_{\tau}$-group structure via the action $(2.7)$ and hence $U(g)$ also admits a $S_{\tau}$-group structure through the restriction of the action (2.7) to $U(g)$. And $H^{1}\left(S_{\tau}, U(g)\right)$ and $H^{1}\left(S_{\tau}, S p(g, \mathbb{R})\right)$ are trivial. 
Proof. The proof can be found in [8, pp. 8-9]. However I will give a sketchy proof for the reader. Assume $f_{k}$ is a 1-cocycle in $Z^{1}\left(S_{\tau}, U(g)\right)$ with $k=$ $\left(\begin{array}{cc}A & B \\ -B & A\end{array}\right) \in U(g)$. Using the fact $\tau(k) k=I_{g}$, we see that

$$
A={ }^{t} A, \quad B={ }^{t} B, \quad A B=B A \quad \text { and } \quad A^{2}+B^{2}=I_{g} .
$$

Therefore we can find $h \in O(g)$ such that $h(A+i B) h^{-1}=\mathfrak{D} \in U(g)$ is diagonal. We take $\mu=\sqrt{\mathfrak{D}} \in U(g)$ by choosing a square root of each diagonal entry. We set $\delta=h^{-1} \mu h$. Then $k=\tau(\delta) \delta^{-1}$. By Lemma 1, $f_{k}$ is a 1-coboundary. Hence $H^{1}\left(S_{\tau}, U(g)\right)$ is trivial.

Let $G=S p(g, \mathbb{R})$ as before. Suppose $f_{M} \in Z^{1}\left(S_{\tau}, G\right)$ with $M=\left(\begin{array}{cc}A & B \\ C & D\end{array}\right) \in G$. Then we see that $f_{M}$ is a 1-coboundary with values in $G$ if and only if $\mathbb{H}_{g}^{M \tau} \neq \emptyset$. We can find $M_{1} \in G$ such that $\mathbb{H}_{g}^{M_{1} \tau} \neq \emptyset$ and $f_{M} \sim f_{M_{1}} \sim f_{b}$. Therefore $f_{M} \sim f_{b}$, that is, $f_{M}$ is a 1-coboundary with values in $G$. Hence $H^{1}\left(S_{\tau}, G\right)$ is trivial.

Theorem C. For all $m \geq 1$, the mapping

$$
H^{1}\left(S_{\tau}, \Gamma_{g}(4 m)\right) \longrightarrow H^{1}\left(S_{\tau}, \Gamma_{g}(2,2 m)\right)
$$

is trivial.

Proof. The proof can be found in [8, pp. 7-10]. We will give a sketchy proof for the reader. In order to prove this theorem, we need the following lemma.

Lemma 3. If $\tau(\gamma) \gamma \in \Gamma_{g}(4 m)$ with $\gamma \in \Gamma_{g}$, then $\gamma=\beta u$ for some $\beta \in$ $\Gamma_{g}(2,2 m)$ and for some $u \in G L(g, \mathbb{Z})$.

Lemma 4. Let $\gamma \in \Gamma_{g}(2)$ and suppose $\Omega \in \mathbb{H}_{g}$ is not fixed by any element of $\Gamma_{g}$ other than $\pm I_{g}$. Suppose $\tau(\Omega)=\gamma \cdot \Omega$. Then there exists an element $h \in \Gamma_{g}$ such that $\gamma=\tau(h) h^{-1}$.

Lemma 4 is a consequence of the theorem of Silhol [26, Theorem 1.4] and Comessatti. Suppose $f_{\gamma}$ is a cocycle in $Z^{1}\left(S_{\tau}, \Gamma_{g}(4 m)\right)$ with $\gamma \in \Gamma_{g}(4 m)$. According to Theorem B, its image in $\left.H^{1}\left(S_{\tau}, G\right)\right)$ is a coboundary and so there exists $h \in G$ with $\gamma=\tau(h) h^{-1}$. Thus $\mathbb{H}^{\tau \gamma}=h \cdot\left(i \mathcal{P}_{g}\right)$. By Lemma 2.2 , there exists $\Omega \in \mathbb{H}^{\tau \gamma}$ which are not fixed by any element of $\Gamma_{g}$ other than $\pm I_{2 g}$ and the set of such points is the complement of a countable union of proper real algebraic subvarieties of $\mathbb{H}^{\tau \gamma}$. According to Lemma $4, \gamma=\tau(h) h^{-1}$ for some $h \in \Gamma_{g}$. By Lemma 3, we may write $h=\beta u$ for some $\beta \in \Gamma_{g}(2,2 m)$ and for some $u \in G L(g, \mathbb{Z})$. Then $\gamma=\tau(h) h^{-1}=\tau(\beta) \beta^{-1}$. By Lemma 1 , the cohomology class $\left[f_{\gamma}\right]$ is trivial in $H^{1}\left(S_{\tau}, \Gamma_{g}(2,2 m)\right)$.

Theorem D. Let $\Gamma_{0}=\Gamma_{g}(2,2 m)$ and $\Gamma=\Gamma_{g}(4 m)$. Then we have the following results:

(a) $\mathbf{D}^{\tau}=G^{\tau} / K^{\tau}$;

(b) For each cohomology class $\left[f_{\gamma}\right] \in H^{1}\left(S_{\tau}, \Gamma\right)$, there exists $h \in \Gamma_{0}$ such that $\gamma=\tau(h) h^{-1}$, in which case

$$
\mathbf{D}^{\tau \gamma}=h \mathbf{D}^{\tau} \quad \text { and } \quad \Gamma^{\tau \gamma}=h \Gamma^{\tau} h^{-1} .
$$


(c) The association $f_{\gamma} \longrightarrow h(c f$. see $(2))$ determines a one-to-one correspondence between $H^{1}\left(S_{\tau}, \Gamma\right)$ and $\Gamma \backslash \Gamma_{0} / \Gamma_{0}^{\tau}$.

(d)

$$
X_{\Gamma}^{\tau}:=\coprod_{h \in \Gamma \backslash \Gamma_{0} / \Gamma_{0}^{\tau}} h \Gamma^{\tau} h^{-1} \backslash h \mathbf{D}^{\tau} .
$$

Proof. (a) follows from the fact that $H^{1}\left(S_{\tau}, U(g)\right)$ is trivial (cf. Theorem B).

(b) follows from Theorem C.

(c) follows from Theorem $\mathrm{C}$, and the facts that $\Gamma$ is a normal subgroup of $\Gamma_{0}$ and that $\tau$ acts on $\Gamma \backslash \Gamma_{0}$ trivially.

(d) follows from Theorem $\mathrm{C}$ and the facts that $\Gamma$ is a normal subgroup of $\Gamma_{0}$ and that $\tau$ acts on $\Gamma \backslash \Gamma_{0}$ trivially together with the fact that $\Gamma \mathrm{s}$ torsion free.

Corollary. Let $m$ be a positive integer with $m \geq 1$. Let $S_{\tau}$ be as in Theorem A. Let $\Gamma=\Gamma_{g}(4 m)$ and $X=\Gamma \backslash \mathbb{H}_{g}$. The set $X_{\mathbb{R}}$ of real points of $X$ is given by

$$
X_{\mathbb{R}}=\coprod_{h} \Gamma_{[h]} \backslash h \cdot\left(i \mathcal{P}_{g}\right)=\Gamma \backslash \mathbb{H}^{\tau \Gamma},
$$

where $h$ is indexed by elements

$$
h \in \Gamma_{g}(4 m) \backslash \Gamma_{g}(2,2 m) / \Gamma_{g}^{[L]}(2)=H^{1}\left(S_{\tau}, \Gamma_{g}(4 m)\right)
$$

and $\Gamma_{[h]}:=h \Gamma_{g}^{[L]}(4 m) h^{-1}$.

Proof. The proof follows from (c) and (d) in Theorem D.

\section{References}

[1] A. A. Albert, Symmetric and alternate matrices in an arbitrary field. I, Trans. Amer. Math. Soc. 43 (1938), no. 3, 386-436.

[2] A. Ash, D. Mumford, M. Rapoport, and Y. Tai, Smooth compactification of locally symmetric varieties, Lie Groups: History, Frontiers and Applications, Vol. IV, Math. Sci. Press, Brookline, Mass., 1975.

[3] W. Baily, Satake's compactification of $V_{n}^{*}$, Amer. J. Math. 80 (1958), 348-364.

[4] W. Baily and A. Borel, Compactification of arithmetic quotients of bounded symmetric domains, Ann. Math. 84 (1966), 442-528.

[5] C. Birkenhake and H. Lange, Complex Tori, Progress in Mathematics, 177. Birkhäuser Boston, Inc., Boston, 1999.

[6] H. Comessati, Sulle varietà abeliane reali. I, II, Ann. Mat. Pura. Appl. 2 (1924), 67-106 and 4 (1926), 27-72.

[7] G. Faltings and C.-L. Chai, Degeneration of Abelian Varieties, Ergebnisse der Math. 22, Springer-Verlag, Berlin-Heidelberg-New York, 1990.

[8] M. Goresky and Y. S. Tai, The moduli space of real abelian varieties with level structure, Compositio Math. 139 (2003), no. 1, 1-27.

[9] S. Helgason, Groups and Geometric Analysis, Academic Press, New York, 1984.

[10] J. Igusa, Theta Functions, Springer-Verlag, Berlin-Heidelberg-New York, 1972.

[11] M. Itoh, On the Yang Problem (SFT), preprint, Max-Planck Institut für Mathematik, Bonn, 2011.

[12] A. W. Knapp, Representation Theory of Semisimple Groups, Princeton University Press, Princeton, New Jersey, 1986. 
[13] H. Lange and C. Birkenhake, Complex Abelian Varieties, Grundlehren der mathematischen Wissenschaften, Springer-Verlag, 1992

[14] H. Maass, Siegel modular forms and Dirichlet series, Lecture Notes in Math. 216, Springer-Verlag, Berlin-Heidelberg-New York, 1971.

[15] Y. Matsushima, On the intermediate cohomology group of a holomorphic line bundle over a complex torus, Osaka J. Math. 16 (1979), no. 3, 617-631.

[16] H. Minkowski, Gesammelte Abhandlungen, Chelsea, New York, 1967.

[17] D. Mumford, Abelian Varieties, Oxford University Press, 1970; Reprinted, 1985.

[18] I. Satake, On the compactification of the Siegel space, J. Indian Math. Soc. 20 (1956), $259-281$.

[19] _ Algebraic Structures of Symmetric Domains, Kano Memorial Lectures 4, Iwanami Shoton, Publishers and Princeton University Press, 1980.

[20] A. Selberg, Harmonic analysis and discontinuous groups in weakly symmetric Riemannian spaces with applications to Dirichlet series, J. Indian Math. Soc. 20 (1956), 47-87.

[21] M. Seppälä and R. Silhol, Moduli spaces for real algebraic curves and real abelian varieties, Math. Z. 201 (1989), no. 2, 151-165.

[22] G. Shimura, On the Fourier coefficients of modular forms of several variables, Nachr. Akad. Wiss. Göttingen Math.-Phys. Kl. II (1975), no. 17, 261-268.

[23] C. L. Siegel, Symplectic geometry, Amer. J. Math. 65 (1943), 1-86; Academic Press, New York and London, 1964; Gesammelte Abhandlungen, no. 41, vol. II, 274-359, Springer-Verlag, 1966

[24] R. Silhol, Real Abelian varieties and the theory of Comessatti, Math. Z. 181 (1982), no. $3,345-364$

[25] _ Real Algebraic Surfaces, Lecture Notes in Math. 1392, Springer-Verlag, BerlinHeidelberg-New York, 1989.

[26] Compactifications of moduli spaces in real algebraic geometry, Invent. Math. 107 (1992), no. 1, 151-202.

[27] J.-H. Yang, A note on holomorphic vector bundles over complex tori, Bull. Korean Math. Soc. 23 (1986), no. 2, 149-154.

[28] Holomorphic vector bundles over complex tori, J. Korean Math. Soc. 26 (1989), no. $1,117-142$

[29] - A note on a fundamental domain for Siegel-Jacobi space, Houston J. Math. 32 (2006), no. 3, 701-712

[30] - Invariant metrics and Laplacians on Siegel-Jacobi space, J. Number Theory 127 (2007), no. 1, 83-102.

[31] A partial Cayley transform of Siegel-Jacobi disk, J. Korean Math. Soc. 45 (2008), no. 3, 781-794.

[32] Remark on harmonic analysis on the Siegel-Jacobi space, arXiv:1107.0509v1 [math.NT], 2009

[33] - Invariant metrics and Laplacians on Siegel-Jacobi disk, Chin. Ann. Math. Ser. B 31 (2010), no. 1, 85-100.

[34] Invariant differential operators on the Siegel-Jacobi space and Maass-Jacobi forms, Proceedings of the International Conference on Geometry, Number Theory and Representation Theory, 39-63, KM Kyung Moon Sa, Seoul, 2013.

[35] _ Invariant differential operators on the Minkowski-Euclid space, J. Korean Math. Soc. 50 (2013), no. 2, 275-306.

Department of Mathematics

INHA UNIVERSITY

INCHEON 402-751, KOREA

E-mail address: jhyang@inha.ac.kr 